\title{
THE SIXTH MOMENT OF AUTOMORPHIC L-FUNCTIONS
}

\author{
VORRAPAN CHANDEE AND XIANNAN LI
}

\begin{abstract}
In this paper, we consider the $L$-functions $L(s, f)$ where $f$ is an eigenform for the congruence subgroup $\Gamma_{1}(q)$. We prove an asymptotic formula for the sixth moment of this family of automorphic $L$-functions.
\end{abstract}

\section{INTRODUCTION}

Moments of $L$-functions are of great interest to analytic number theorists. For instance, for $\zeta(s)$ denoting the Riemann zeta function and

$$
I_{k}(T):=\int_{0}^{T}\left|\zeta\left(\frac{1}{2}+i t\right)\right|^{2 k} d t,
$$

asymptotic formulae were proven for $k=1$ by Hardy and Littlewood and for $k=2$ by Ingham (see [21] VII). This work is closely related to zero density results and the distribution of primes in short intervals. More recently, moments of other families of $L$-functions for studied for their numerous applications, including non-vanishing and subconvexity results. In many applications, it is important to develop technology which can understand such moments for larger $k$.

The behavior of moments for larger $k$ remain mysterious. However, recently there has been great progress in our understanding. First, good heuristics and conjectures on the behavior of $I_{k}(T)$ appeared in the literature. To be precise, a folklore conjecture states that

$$
I_{k}(T) \sim c_{k} T(\log T)^{k^{2}}
$$

for constants $c_{k}$ depending on $k$ but the values of $c_{k}$ were unknown for general $k$ until the work of Keating and Snaith [15] which related these moments to circular unitary ensembles and provided precise conjectures for $c_{k}$. The choice of group is consistent with the Katz-Sarnak philosophy [14], which indicates that the symmetry group associated to this family should be unitary. Based on heuristics for shifted divisor sums, Conrey and Ghosh derived a conjecture in the case $k=3[3]$ and Conrey and Gonek derived a conjecture in the case $k=4$ [4]. In particular, the conjecture for the sixth moment is

$$
I_{3}(T) \sim 42 a_{3} \frac{T(\log T)^{9}}{9 !}
$$

for some arithmetic factor $a_{3}$. Further conjectures including lower order terms, and for other symmetry groups are available from the work of Conrey, Farmer, Keating,

Date: March 21, 2018.

2010 Mathematics Subject Classification. 11M41, 11F11, 11F67.

Key words and phrases. moment of $L$-functions, automorphic $L$-functions, $\Gamma_{1}(q)$. 
Rubinstein and Snaith [2] as well as from the work of Diaconu, Goldfeld and Hoffstein [8].

In support of these conjectures, lower bounds of the the right order of magnitude are available due to Rudnick and Soundararajan [17], while good upper bounds of the right order of magnitude are available conditionally on RH, due to Soundararajan [19] and later improved by Harper [10].

Despite this, verifications of the moment conjectures for high moments remain elusive. Typically, even going slightly beyong the fourth moment to obtain a twisted fourth moment is quite difficult, and there are few families for which this is known.

Quite recently, Conrey, Iwaniec and Soundararajan [5] derived an asymptotic formula for the sixth moment of Dirichlet $L$-functions with a power saving error term. Instead of fixing the modulus $q$ and only averaging over primitive characters $\chi \bmod q$, they also average over the modulus $q \leq Q$, which gives them a larger family of size $Q^{2}$. Further, they include a short average on the critical line. In particular, they showed that

$$
\sum_{q \leq Q} \sum_{\chi(\bmod q)}^{*} \int_{-\infty}^{\infty}\left|L\left(\frac{1}{2}+i t, \chi\right)\right|^{6}\left|\Gamma\left(\frac{1}{4}+\frac{i t}{2}\right)\right|^{6} d t \sim 42 b_{3} \frac{Q^{2}(\log Q)^{9}}{9 !} \int_{-\infty}^{\infty}\left|\Gamma\left(\frac{1}{4}+\frac{i t}{2}\right)\right|^{6} d t
$$

for some constant $b_{3}$. This is consistent with the analogous conjecture for the Riemann zeta function above.

The authors of this paper subsequently derived an asymptotic formula for the eight moment of this family of $L$-functions, conditionally on GRH [1], which is

$\sum_{q \leq Q} \sum_{\chi(\bmod q)}^{*} \int_{-\infty}^{\infty}\left|L\left(\frac{1}{2}+i t, \chi\right)\right|^{8}\left|\Gamma\left(\frac{1}{4}+\frac{i t}{2}\right)\right|^{8} d t \sim 24024 b_{4} \frac{Q^{2}(\log Q)^{16}}{16 !} \int_{-\infty}^{\infty}\left|\Gamma\left(\frac{1}{4}+\frac{i t}{2}\right)\right|^{8} d t$

for some constant $b_{4}$.

In this paper, we study a family of $L$-functions attached to automorphic forms on $G L(2)$. To be more precise, let $S_{k}\left(\Gamma_{0}(q), \chi\right)$ be the space of cusp forms of weight $k \geq 2$ for the group $\Gamma_{0}(q)$ and the nebentypus character $\chi(\bmod q)$, where

$$
\Gamma_{0}(q)=\left\{\left(\begin{array}{ll}
a & b \\
c & d
\end{array}\right) \mid a d-b c=1, \quad c \equiv 0(\bmod q)\right\} .
$$

Also, let $S_{k}\left(\Gamma_{1}(q)\right)$ be the space of holomorphic cusp forms for the group

$$
\Gamma_{1}(q)=\left\{\left(\begin{array}{ll}
a & b \\
c & d
\end{array}\right) \mid a d-b c=1, \quad c \equiv 0(\bmod q), \quad a \equiv d \equiv 1(\bmod q)\right\} .
$$

Note that $S_{k}\left(\Gamma_{1}(q)\right)$ is a Hilbert space with the Petersson's inner product

$$
<f, g>=\int_{\Gamma_{1}(q) \backslash \mathbb{H}} f(z) \bar{g}(z) y^{k-2} d x d y,
$$

and

$$
S_{k}\left(\Gamma_{1}(q)\right)=\bigoplus_{\chi(\bmod q)} S_{k}\left(\Gamma_{0}(q), \chi\right)
$$


Let $\mathcal{H}_{\chi} \subset S_{k}\left(\Gamma_{0}(q), \chi\right)$ be an orthogonal basis of $S_{k}\left(\Gamma_{0}(q), \chi\right)$ consisting of Hecke cusp forms, normalized so that the first Fourier coefficient is 1 . For each $f \in \mathcal{H}_{\chi}$, we let $L(f, s)$ be the $L$-function associated to $f$, defined for $\operatorname{Re}(s)>1$ as

$$
L(f, s)=\sum_{n \geq 1} \frac{\lambda_{f}(n)}{n^{s}}=\prod_{p}\left(1-\frac{\lambda_{f}(p)}{p^{s}}+\frac{\chi(p)}{p^{2 s}}\right)^{-1},
$$

where $\left\{\lambda_{f}(n)\right\}$ are the Hecke eigenvalues of $f$. With our normalization, $\lambda_{f}(1)=1$. In general, the Hecke eigenvalues satisfy the Hecke relation

$$
\lambda_{f}(m) \lambda_{f}(n)=\sum_{d \mid(m, n)} \chi(d) \lambda_{f}\left(\frac{m n}{d^{2}}\right),
$$

for all $m, n \geq 1$. We define the completed $L$-function as

$$
\Lambda\left(f, \frac{1}{2}+s\right)=\left(\frac{q}{4 \pi^{2}}\right)^{\frac{s}{2}} \Gamma\left(s+\frac{k}{2}\right) L\left(f, \frac{1}{2}+s\right),
$$

which satisfies the functional equation

$$
\Lambda\left(f, \frac{1}{2}+s\right)=i^{k} \bar{\eta}_{f} \Lambda\left(\bar{f}, \frac{1}{2}-s\right),
$$

where $\left|\eta_{f}\right|=1$ when $f$ is a newform.

Suppose for each $f \in \mathcal{H}_{\chi}$, we have an associated number $\alpha_{f}$. Then we define the harmonic average of $\alpha_{f}$ over $\mathcal{H}_{\chi}$ to be

$$
\sum_{f \in \mathcal{H}_{\chi}}^{h} \alpha_{f}=\frac{\Gamma(k-1)}{(4 \pi)^{k-1}} \sum_{f \in \mathcal{H}_{\chi}} \frac{\alpha_{f}}{\|f\|^{2}} .
$$

We note that when the first coefficient $\lambda_{f}(1)=1,\|f\|^{2}$ is essentially the value of a certain $L$-function at 1 , and so on average, $\|f\|^{2}$ is constant. As in other works, it is possible to remove the weighting by $\|f\|^{2}$ through what is now a standard argument.

We shall be interested in moments of the form

$$
\frac{2}{\phi(q)} \sum_{\substack{\chi(\bmod q) \\ \chi(-1)=(-1)^{k}}} \sum_{f \in \mathcal{H}_{\chi}}^{h}|L(f, 1 / 2)|^{2 k} .
$$

We note that the size of the family is around size $q^{2}$. For prime level, $\eta_{f}$ can be expressed in terms of Gauss sums, and in particular we expect $\eta_{f}$ to equidistribute on the circle as $f$ varies over an orthogonal basis of $S_{k}\left(\Gamma_{1}(q)\right)$. Thus, we expect our family of $L$-functions to be unitary.

In this paper, we prove an asymptotic formula for the sixth moment - this will be the first time that the sixth moment of a family of $L$-functions over $G L(2)$ has been understood. Following [2], we have the following conjecture for the sixth moment of our family. We refer the reader to Appendix A.1 for a brief derivation of the arithmetic factor in the conjecture. 
Conjecture 1.1. Let $q$ be a prime number. As $q \rightarrow \infty$, we have

$$
\frac{2}{\phi(q)} \sum_{\substack{\chi(\bmod q) \\ \chi(-1)=(-1)^{k}}} \sum_{f \in \mathcal{H}_{\chi}}^{h}|L(f, 1 / 2)|^{6} \sim 42 \mathscr{C}_{3}\left(1-\frac{1}{q}\right)^{4}\left(1+\frac{4}{q}+\frac{1}{q^{2}}\right) C_{q}^{-1} \frac{(\log q)^{9}}{9 !}
$$

where

$$
\mathscr{C}_{3}:=\prod_{p} C_{p}, \quad \text { and }, \quad C_{p}:=\left(1+\frac{4}{p}+\frac{7}{p^{2}}-\frac{2}{p^{3}}+\frac{9}{p^{4}}-\frac{16}{p^{5}}+\frac{1}{p^{6}}-\frac{4}{p^{7}}\right)\left(1+\frac{1}{p}\right)^{-4} .
$$

Iwaniec and Xiaoqing Li proved a large sieve result for this family in [13], and Djankovic used their result to prove [7] that for an odd integer $k \geq 3$ and prime $q$ that

$$
\frac{2}{\phi(q)} \sum_{\substack{\chi(\bmod \\ \chi(-1)=-1}} \sum_{f \in \mathcal{H}_{\chi}}^{h}|L(f, 1 / 2)|^{6} \ll q^{\varepsilon} .
$$

as $q \rightarrow \infty$. In this paper, we shall prove the following

Theorem 1.2. Let $q$ be a prime and $k \geq 5$ be odd. Then, as $q \rightarrow \infty$, we have

$$
\begin{aligned}
\frac{2}{\phi(q)} \sum_{\substack{\chi(\bmod \\
\chi(-1)=-1}} \sum_{f \in \mathcal{H}_{\chi}}^{h} \int_{-\infty}^{\infty}\left|\Lambda\left(f, \frac{1}{2}+i t\right)\right|^{6} d t \\
\quad \sim 42 \mathscr{C}_{3}\left(1-\frac{1}{q}\right)^{4}\left(1+\frac{4}{q}+\frac{1}{q^{2}}\right) C_{q}^{-1} \frac{(\log q)^{9}}{9 !} \int_{-\infty}^{\infty}\left|\Gamma\left(\frac{k}{2}+i t\right)\right|^{6} d t,
\end{aligned}
$$

where $\mathscr{C}_{3}$ and $C_{p}$ are defined in (1.4).

In fact, we are able to prove this with an error term of $q^{-1 / 4}$, as opposed to the $q^{-1 / 10}$ error term in the work of Conrey, Iwaniec and Soundararajan[5]. The reason behind this superior error term is explained in the outline in Section 1.1. In future work, we hope to extend our attention to the eighth momment.

The assumption that $k$ is odd implies that all $f \in \mathcal{H}_{\chi}$ are newforms. This is for convenience only and is not difficult to remove. Indeed, when $k$ is even, all $f \in \mathcal{H}_{\chi}$ are newforms except possibly when $\chi$ is the principal character and $f$ is induced by a cusp form of full level. We avoid this case for the sake of brevity. Similarly, the assumption that $k \geq 5$ simplifies parts of the calculation; it is possible to prove Theorem 1.2 for smaller $k$.

Since the $\Gamma$ function decays rapidly on vertical lines, the average over $t$ is fairly short. It is included for the same reason as in the works [5] and [1] in that it allows us to avoid certain unbalanced sums in the computation of the moment. Although this appears to be a small technical change in the main statement, evaluating such moments without the short integration over $t$ is a significant challenge. Our Theorem will follow from the more general Theorem 2.5 for shifted moments in Section 2. 
1.1. Outline of paper. To help orient the reader, we provide a sketch of the proof, and introduce the various sections of the paper. After applying the approximate functional equation developed in Section 3, the main object to be understood is roughly of the form

$$
\frac{2}{\phi(q)} \sum_{\substack{\chi(\bmod q) \\ \chi(-1)=(-1)^{k}}} \sum_{f \in \mathcal{H}_{\chi}}^{h} \sum_{m, n \asymp q^{3 / 2}} \frac{\sigma_{3}(m) \sigma_{3}(n) \lambda_{f}(n) \overline{\lambda_{f}}(m)}{\sqrt{m n}} .
$$

In fact, since the coefficients $\lambda_{f}(n)$ are not completely multiplicative, the expression is significantly more complicated for the purpose of extracting main terms.

Applying Peterson's formula for the average over $f \in \mathcal{H}_{\chi}$ leads to diagonal terms $m=n$ which are evaluated fairly easily in Section 4.1 as well as off-diagonal terms which involve sums of the form

$$
\sum_{m, n \asymp q^{3 / 2}} \frac{\sigma_{3}(m) \sigma_{3}(n)}{\sqrt{m n}} \frac{2}{\phi(q)} \sum_{\substack{\chi(\bmod q) \\ \chi(-1)=(-1)^{k}}} \sum_{c} S_{\chi}(m, n ; c q) J_{k-1}\left(\frac{4 \pi \sqrt{m n}}{c q}\right),
$$

where $S_{\chi}(m, n ; c q)$ is the Kloosterman sum defined in $(2.12)$, and $J_{k-1}(x)$ is the J-Bessel function of order $k-1$.

Let us focus on the transition region for the Bessel function where $c \asymp q^{1 / 2}$, so that the conductor is a priori of size $q c \asymp q^{3 / 2}$. It is here that the addition average over $\chi \bmod q$ comes into play. To be more precise, to understand the exponential sum

$$
\frac{2}{\phi(q)} \sum_{\substack{\chi(\bmod q) \\ \chi(-1)=(-1)^{k}}} S_{\chi}(m, n ; c q)
$$

it suffices to understand

$$
\sum_{\substack{a \bmod c q \\ a \equiv 1 \bmod q}}^{*} e\left(\frac{a m+\bar{a} n}{c q}\right)
$$

which, assuming that $(c, q)=1$, is

$$
e\left(\frac{m+n}{c q}\right) \sum_{a \bmod c}^{*} e\left(\frac{\bar{q}(a-1) m+\bar{q}(\bar{a}-1) n}{c}\right)
$$

using Chinese remainder theorem and reciprocity. The factor $e\left(\frac{m+n}{c q}\right)$ has small derivatives and may be treated as a smooth function, while the conductor of the rest of the exponential sum has decreased to $c \asymp q^{1 / 2}$. The details of these calculations are in Section 5 .

This phenomenon of the drop in conductor appears in other examples. In the case of the sixth moment of Dirichlet $L$-functions in [5], it occurs when replacing $q$ with the complementary divisor $\frac{m-n}{q} \asymp q^{1 / 2}$. It is quite interesting that the same drop in conductor occurs by seemingly very different mechanisms. However, note that when the complementary divisor is small, the ordered pair $(m, n)$ is forced to be in a narrow 
region. That this does not occur in our case is one of reasons behind the superior error term in our result; the assumption that $q$ is prime also plays a role.

After the conductor drop, we apply Voronoi summation to the sum over $m$ and $n$ in $\S 6$. We need a version of Voronoi summation including shifts. The proof of this is essentially the same as the proof of the standard Voronoi summation formula for $\sigma_{3}(n)$ by Ivic [11]. We state the result required in Appendix B.

After applying Voronoi, it is easy to guess which terms should contribute to the main terms and which terms should be error terms. The main terms are described in Proposition 6.1 and the error terms are bounded in Proposition 6.2. Essentially, we expect the main terms to be a sum of products of 9 factors of $\zeta$, the same as the diagonal contribution but with permutations in the shifts, as in Theorem 2.5. This is by no means immediately visible from the expression in Proposition 6.1. Indeed, it takes some effort to see that we get the right number of $\zeta$ factors. Along the way, we use, among other things, a calculation of Iwaniec and Xiaoqing Li in [13]. This is done in Section 7. In order to finish the verifications, we need to check that the local factors of two expressions agree. The details here are standard but intricate, and are provided in Appendix A.

Finally, the error terms from Voronoi summation are bounded in Section 8. Here, one needs to show that the dual sums from Voronoi summation are essentially quite short, which is related to the reduction in conductor from $c q$ to $c$ earlier.

\section{Notation AND THE SHIfTED SiXth MOMENT}

We begin with some notation. Let $\boldsymbol{\alpha}:=\left(\alpha_{1}, \alpha_{2}, \alpha_{3}\right)$ and $\boldsymbol{\beta}:=\left(\beta_{1}, \beta_{2}, \beta_{3}\right)$. For a complex number $s$, we shall write $\boldsymbol{\alpha}+s:=\left(\alpha_{1}+s, \alpha_{2}+s, \alpha_{3}+s\right)$. We define

$$
\begin{gathered}
\delta(\boldsymbol{\alpha}, \boldsymbol{\beta}):=\frac{1}{2} \sum_{j=1}^{3}\left(\alpha_{j}-\beta_{j}\right), \\
G(s ; \boldsymbol{\alpha}, \boldsymbol{\beta}):=\prod_{j=1}^{3} \Gamma\left(s+\frac{k-1}{2}+\alpha_{j}\right) \Gamma\left(s+\frac{k-1}{2}-\beta_{j}\right),
\end{gathered}
$$

and

$$
\Lambda(f, s ; \boldsymbol{\alpha}, \boldsymbol{\beta}):=\prod_{j=1}^{3} \Lambda\left(f, s+\alpha_{j}\right) \Lambda\left(\bar{f}, s-\beta_{j}\right) .
$$

Note that we have

$$
\Lambda(f ; \boldsymbol{\alpha}, \boldsymbol{\beta})=\Lambda\left(f, \frac{1}{2} ; \boldsymbol{\alpha}, \boldsymbol{\beta}\right)=\left(\frac{q}{4 \pi^{2}}\right)^{\delta(\boldsymbol{\alpha}, \boldsymbol{\beta})} G\left(\frac{1}{2} ; \boldsymbol{\alpha}, \boldsymbol{\beta}\right) \prod_{j=1}^{3} L\left(f, \frac{1}{2}+\alpha_{j}\right) L\left(\bar{f}, \frac{1}{2}-\beta_{j}\right) .
$$

We define the shifted $k$-divisor function by

$$
\sigma_{k}\left(n ; \alpha_{1}, \ldots, \alpha_{k}\right)=\sum_{n_{1} n_{2} \ldots n_{k}=n} n_{1}^{-\alpha_{1}} n_{2}^{-\alpha_{2}} \ldots n_{k}^{-\alpha_{k}} .
$$


Let

$$
\mathscr{B}(a, b ; \boldsymbol{\alpha}):=\frac{\mu(a) \sigma_{3}\left(b ; \alpha_{1}+\alpha_{2}, \alpha_{2}+\alpha_{3}, \alpha_{3}+\alpha_{1}\right)}{a^{\alpha_{1}+\alpha_{2}+\alpha_{3}}}
$$

Next we need the following lemmas, which help us generate the conjecture of the sixth moment, namely

$$
\frac{2}{\phi(q)} \sum_{\substack{\chi(\bmod q) \\ \chi(-1)=(-1)^{k}}} \sum_{f \in \mathcal{H}_{\chi}}^{h} \Lambda(f ; \boldsymbol{\alpha}, \boldsymbol{\beta}) .
$$

Lemma 2.1. We have

$$
\sigma_{2}\left(n_{1} n_{2} ; \alpha_{1}, \alpha_{2}\right)=\sum_{d \mid\left(n_{1}, n_{2}\right)} \mu(d) d^{-\alpha_{1}-\alpha_{2}} \sigma_{2}\left(\frac{n_{1}}{d} ; \alpha_{1}, \alpha_{2}\right) \sigma_{2}\left(\frac{n_{2}}{d} ; \alpha_{1}, \alpha_{2}\right)
$$

Proof. Since both sides are multiplicative functions, it is enough to prove the Lemma when $n_{1} n_{2}$ is a prime power. We set $n_{1}=p^{a}$ and $n_{2}=p^{b}$, where $1 \leq a \leq b$. Then

$$
\begin{aligned}
\sigma_{2}\left(p^{a} p^{b} ; \alpha_{1}, \alpha_{2}\right) & =\sum_{0 \leq k \leq a} \mu\left(p^{k}\right) p^{-k\left(\alpha_{1}+\alpha_{2}\right)} \sigma_{2}\left(p^{a-k} ; \alpha_{1}, \alpha_{2}\right) \sigma_{2}\left(p^{b-k} ; \alpha_{1}, \alpha_{2}\right) \\
& =\sigma_{2}\left(p^{a} ; \alpha_{1}, \alpha_{2}\right) \sigma_{2}\left(p^{b} ; \alpha_{1}, \alpha_{2}\right)-p^{-\left(\alpha_{1}+\alpha_{2}\right)} \sigma_{2}\left(p^{a-1} ; \alpha_{1}, \alpha_{2}\right) \sigma_{2}\left(p^{b-1} ; \alpha_{1}, \alpha_{2}\right) .
\end{aligned}
$$

On the other hand,

$$
\sigma_{2}\left(p^{n} ; \alpha_{1}, \alpha_{2}\right)=\sum_{\ell=0}^{n} p^{-\ell \alpha_{1}} p^{-(n-\ell) \alpha_{2}}=p^{-n \alpha_{2}} \frac{1-\frac{1}{p^{(n+1)\left(\alpha_{1}-\alpha_{2}\right)}}}{1-\frac{1}{p^{\alpha_{1}-\alpha_{2}}}}
$$

and the lemma follows by substituting the above formula into (2.8).

We write the product of $L$-functions in term of Dirichlet series in the following lemma

Lemma 2.2. Let $L(f, w)$ be an L-function in $\mathcal{H}_{\chi}$. For $\operatorname{Re}\left(s+\alpha_{i}\right)>1$, we have

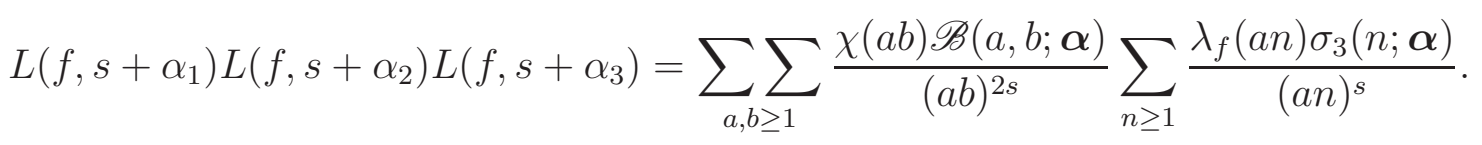


Proof. From the Hecke relation (1.2) and Lemma 2.1, we have

$$
\begin{aligned}
& L\left(f, s+\alpha_{1}\right) L\left(f, s+\alpha_{2}\right) L\left(f, s+\alpha_{3}\right) \\
& =\sum_{n_{1} \geq 1} \frac{\lambda_{f}\left(n_{1}\right)}{n_{1}^{s+\alpha_{1}}} \sum_{d \geq 1} \frac{\chi(d)}{d^{2 s+\alpha_{2}+\alpha_{3}}} \sum_{j \geq 1} \frac{\lambda_{f}(j) \sigma_{2}\left(j ; \alpha_{2}, \alpha_{3}\right)}{j^{s}} \\
& =\sum_{d \geq 1} \frac{\chi(d)}{d^{2 s+\alpha_{2}+\alpha_{3}}} \sum_{n_{1}, j \geq 1} \sum_{\sigma_{2}\left(j ; \alpha_{2}, \alpha_{3}\right)} \sum_{n^{s+\alpha_{1}} j^{s}} \chi(e) \lambda_{f}\left(\frac{j n_{1}}{e^{2}}\right) \\
& =\sum_{a \geq 1} \frac{\mu(a) \chi(a)}{a^{2 s+\alpha_{1}+\alpha_{2}+\alpha_{3}}} \sum_{d, e \geq 1} \sum_{\frac{\chi}{}(d e) \sigma_{2}\left(e ; \alpha_{2}, \alpha_{3}\right)} \sum_{j e)^{2 s} d^{\alpha_{2}+\alpha_{3}} e^{\alpha_{1}}} \sum_{j, n_{1} \geq 1} \frac{\lambda_{f}\left(n_{1} j a\right) \sigma_{2}\left(j ; \alpha_{2}, \alpha_{3}\right)}{\left(a j n_{1}\right)^{s} n_{1}^{\alpha_{1}}} \\
& =\sum_{a \geq 1} \frac{\mu(a) \chi(a)}{a^{2 s+\alpha_{1}+\alpha_{2}+\alpha_{3}}} \sum_{b \geq 1} \frac{\chi(b) \sigma_{3}\left(b ; \alpha_{1}+\alpha_{2}, \alpha_{2}+\alpha_{3}, \alpha_{3}+\alpha_{1}\right)}{b^{2 s}} \frac{\lambda_{f}(a n) \sigma_{3}\left(n ; \alpha_{1}, \alpha_{2}, \alpha_{3}\right)}{(a n)^{s}} .
\end{aligned}
$$

This completes the lemma.

Lemma 2.3. The orthogality relation for Dirichlet characters is

$$
\frac{2}{\phi(q)} \sum_{\substack{\chi(\bmod q) \\ \chi(-1)=(-1)^{k}}} \chi(m) \bar{\chi}(n)= \begin{cases}1 & \text { if } m \equiv n(\bmod q), \quad(m n, q)=1 \\ (-1)^{k} & \text { if } m \equiv-n(\bmod q), \quad(m n, q)=1 \\ 0 & \text { otherwise. }\end{cases}
$$

Petersson's formula gives

$$
\sum_{f \in \mathcal{H}_{\chi}}^{h} \bar{\lambda}_{f}(m) \lambda_{f}(n)=\delta_{m=n}+\sigma_{\chi}(m, n),
$$

where

$$
\begin{aligned}
\sigma_{\chi}(m, n) & =2 \pi i^{-k} \sum_{c \equiv 0(\bmod q)} c^{-1} S_{\chi}(m, n ; c) J_{k-1}\left(\frac{4 \pi}{c} \sqrt{m n}\right) \\
& =2 \pi i^{-k} \sum_{c=1}^{\infty}(c q)^{-1} S_{\chi}(m, n ; c q) J_{k-1}\left(\frac{4 \pi}{c q} \sqrt{m n}\right),
\end{aligned}
$$

and $S_{\chi}$ is the Kloosterman sum defined by

$$
S_{\chi}(m, n ; c q)=\sum_{a \bar{a} \equiv 1} \chi(a) e\left(\frac{a m+\bar{a} n}{c q}\right) .
$$

From Lemma 2.2, we have that

$$
\begin{gathered}
\prod_{i=1}^{3} L\left(f, s+\alpha_{i}\right) L\left(f, s-\beta_{i}\right)=\sum \sum_{a_{1}, b_{1}, a_{2}, b_{2} \geq 1} \sum_{1} \frac{\chi\left(a_{1} b_{1}\right) \bar{\chi}\left(a_{2} b_{2}\right) \mathscr{B}\left(a_{1}, b_{1} ; \boldsymbol{\alpha}\right) \mathscr{B}\left(a_{2}, b_{2} ;-\boldsymbol{\beta}\right)}{\left(a_{1} b_{1} a_{2} b_{2}\right)^{2 s}} \\
\times \sum_{n, m \geq 1} \sum_{\frac{\lambda_{f}}{}\left(a_{1} n\right) \overline{\lambda_{f}}\left(a_{2} m\right) \sigma_{3}(n ; \boldsymbol{\alpha}) \sigma_{3}(m ;-\boldsymbol{\beta})}^{\left(a_{1} n a_{2} m\right)^{s}} .
\end{gathered}
$$


By the orthogonality relation of Dirichlet characters and Petersson's formula in Lemma 2.3, a naive guess might be that the main contribution comes from the diagonal terms $a_{1} b_{1}=a_{2} b_{2}$ and $a_{1} n=a_{2} m$, where $\left(a_{i} b_{i}, q\right)=1$, which is

$$
\mathcal{C}(s, \boldsymbol{\alpha}, \boldsymbol{\beta}):=\sum \sum_{\substack{a_{1}, b_{1}, a_{2}, b_{2}, m, n \geq 1 \\ a_{1} n=a_{2} m \\ a_{1} b_{1}=a_{2} b_{2} \\\left(a_{i}, q\right)=\left(b_{j}, q\right)=1}} \sum \frac{\mathscr{B}\left(a_{1}, b_{1} ; \boldsymbol{\alpha}\right)}{\left(a_{1} b_{1}\right)^{2 s}} \frac{\mathscr{B}\left(a_{2}, b_{2} ;-\boldsymbol{\beta}\right)}{\left(a_{2} b_{2}\right)^{2 s}} \frac{\sigma_{3}(n ; \boldsymbol{\alpha}) \sigma_{3}(m ;-\boldsymbol{\beta})}{\left(a_{1} n\right)^{s}\left(a_{2} m\right)^{s}}
$$

for Re $(s)$ large enough. This can be written as the Euler product

$$
\mathcal{C}(s, \boldsymbol{\alpha}, \boldsymbol{\beta})=\prod_{p} \mathcal{C}_{p}(s, \boldsymbol{\alpha}, \boldsymbol{\beta}),
$$

where for $p \neq q$,

$$
\mathcal{C}_{p}(s, \boldsymbol{\alpha}, \boldsymbol{\beta}):=\sum \sum_{\substack{r_{1}, t_{1}, r_{2}, t_{2}, u_{1}, u_{2} \geq 0 \\ r_{1}+u_{1}=r_{2}+u_{2} \\ r_{1}+t_{1}=r_{2}+t_{2}}} \sum \frac{\mathscr{B}\left(p^{r_{1}}, p^{t_{1}} ; \boldsymbol{\alpha}\right)}{p^{2 s\left(r_{1}+t_{1}\right)}} \frac{\mathscr{B}\left(p^{r_{2}}, p^{t_{2}} ;-\boldsymbol{\beta}\right)}{p^{2 s\left(r_{2}+t_{2}\right)}} \frac{\sigma_{3}\left(p^{u_{1}} ; \boldsymbol{\alpha}\right) \sigma_{3}\left(p^{u_{2}} ;-\boldsymbol{\beta}\right)}{p^{s\left(r_{1}+u_{1}\right)} p^{s\left(r_{2}+u_{2}\right)}},
$$

and for $p=q$,

$$
\mathcal{C}_{q}(s, \boldsymbol{\alpha}, \boldsymbol{\beta}):=\sum_{u \geq 0} \frac{\sigma_{3}\left(q^{u} ; \boldsymbol{\alpha}\right) \sigma_{3}\left(q^{u} ;-\boldsymbol{\beta}\right)}{q^{2 u s}} .
$$

Next, for $\zeta_{p}(w):=\left(1-\frac{1}{p^{w}}\right)^{-1}$, let

$$
\mathcal{Z}_{p}(s ; \boldsymbol{\alpha}, \boldsymbol{\beta}):=\prod_{i=1}^{3} \prod_{j=1}^{3} \zeta_{p}\left(2 s+\alpha_{i}-\beta_{j}\right), \quad \mathcal{Z}(s ; \boldsymbol{\alpha}, \boldsymbol{\beta}):=\prod_{i=1}^{3} \prod_{j=1}^{3} \zeta\left(2 s+\alpha_{i}-\beta_{j}\right) .
$$

and

$$
\mathcal{A}(s ; \boldsymbol{\alpha}, \boldsymbol{\beta}):=\mathcal{C}(s ; \boldsymbol{\alpha}, \boldsymbol{\beta}) \mathcal{Z}(s ; \boldsymbol{\alpha}, \boldsymbol{\beta})^{-1}=\prod_{p} \mathcal{C}_{p}(s ; \boldsymbol{\alpha}, \boldsymbol{\beta}) \mathcal{Z}_{p}(s ; \boldsymbol{\alpha}, \boldsymbol{\beta})^{-1}
$$

We define

$$
\mathcal{M}(q ; \boldsymbol{\alpha}, \boldsymbol{\beta}):=\left(\frac{q}{4 \pi^{2}}\right)^{\delta(\boldsymbol{\alpha}, \boldsymbol{\beta})} G\left(\frac{1}{2} ; \boldsymbol{\alpha}, \boldsymbol{\beta}\right) \mathcal{A} \mathcal{Z}\left(\frac{1}{2} ; \boldsymbol{\alpha}, \boldsymbol{\beta}\right) .
$$

When $\operatorname{Re}\left(\alpha_{i}\right), \operatorname{Re}\left(\beta_{i}\right) \ll \frac{1}{\log q}$, the term $\mathcal{A}\left(\frac{1}{2}, \boldsymbol{\alpha}, \boldsymbol{\beta}\right)$ is absolutely convergent. Now, let $S_{j}$ be the permutation group of $j$ variables. Based on the analysis of the diagonal contribution, we expect $\mathcal{M}(q ; \boldsymbol{\alpha}, \boldsymbol{\beta})$ to be a part of the average in $(2.7)$, and we also notice that the expression $\mathcal{M}(q ; \boldsymbol{\alpha}, \boldsymbol{\beta})$ is fixed by the action of $S_{3} \times S_{3}$. Since we expect our final answer to be symmetric under the full group $S_{6}$, we sum over the cosets $S_{6} /\left(S_{3} \times S_{3}\right)$. In fact, the method of Conrey, Farmer, Keating, Rubinstein and Snaith [2] gives the following conjecture for the average of $\Lambda(f ; \boldsymbol{\alpha}, \boldsymbol{\beta})$. 
Conjecture 2.4. Assume that $\boldsymbol{\alpha}, \boldsymbol{\beta}$ satisfy $\operatorname{Re}\left(\alpha_{i}\right), \operatorname{Re}\left(\beta_{i}\right) \ll \frac{1}{\log q}$ and $\operatorname{Im}\left(\alpha_{i}\right), \operatorname{Im}\left(\beta_{i}\right) \ll$ $q^{1-\varepsilon}$. We have

$$
\frac{2}{\phi(q)} \sum_{\substack{\chi(\bmod q) \\ \chi(-1)=(-1)^{k}}} \sum_{f \in \mathcal{H}_{\chi}}^{h} \Lambda(f ; \boldsymbol{\alpha}, \boldsymbol{\beta})=\sum_{\pi \in S_{6} /\left(S_{3} \times S_{3}\right)} \mathcal{M}(q ; \pi(\boldsymbol{\alpha}, \boldsymbol{\beta}))\left(1+O\left(q^{-\frac{1}{2}+\varepsilon}\right)\right),
$$

where we define $\pi(\boldsymbol{\alpha}, \boldsymbol{\beta})=\pi\left(\alpha_{1}, \ldots, \alpha_{k}, \beta_{1}, \ldots, \beta_{k}\right)$ for $\pi \in S_{2 k}$, where $\pi$ acts on the $2 k$ tuple $\left(\alpha_{1}, \ldots, \alpha_{k}, \beta_{1}, \ldots, \beta_{k}\right)$ as usual.

We will also write $\pi(\boldsymbol{\alpha}, \boldsymbol{\beta})=(\pi(\boldsymbol{\alpha}), \pi(\boldsymbol{\beta}))$ by an abuse of notation, where $\pi(\boldsymbol{\alpha}, \boldsymbol{\beta})$ is as above. Our main goal is to find an asymptotic formula for

$$
\mathcal{M}_{6}(q):=\frac{2}{\phi(q)} \sum_{\substack{\chi(\bmod q) \\ \chi(-1)=(-1)^{k}}} \sum_{f \in \mathcal{H}_{\chi}}^{h} \int_{-\infty}^{\infty} \Lambda(f ; \boldsymbol{\alpha}+i t, \boldsymbol{\beta}+i t) d t
$$

and we will prove the following result.

Theorem 2.5. Let $q$ be prime and $k \geq 5$ be odd. For $\alpha_{i}, \beta_{j} \ll \frac{1}{\log q}$, we have that

$$
\mathcal{M}_{6}(q)=\int_{-\infty}^{\infty} \sum_{\pi \in S_{6} /\left(S_{3} \times S_{3}\right)} \mathcal{M}(q, \pi(\boldsymbol{\alpha})+i t, \pi(\boldsymbol{\beta})+i t) d t+O\left(q^{-\frac{1}{4}+\varepsilon}\right) .
$$

We note that as the shifts go to 0 , the main term of this moment is of the size $(\log q)^{9}$, and we derive Theorem (1.2). We refer the reader to [2] for the details of this type of calculation.

\section{Approximate functional equation}

In this section, we will prove an approximate functional equation for the product of $L$-functions. Let

$$
H(s ; \boldsymbol{\alpha}, \boldsymbol{\beta})=\prod_{j=1}^{3} \prod_{\ell=1}^{3}\left(s^{2}-\left(\frac{\alpha_{j}-\beta_{\ell}}{2}\right)^{2}\right)^{3}
$$

and define for any $\xi>0$,

$$
W(\xi ; \boldsymbol{\alpha}, \boldsymbol{\beta})=\frac{1}{2 \pi i} \int_{(1)} G\left(\frac{1}{2}+s ; \boldsymbol{\alpha}, \boldsymbol{\beta}\right) H(s ; \boldsymbol{\alpha}, \boldsymbol{\beta}) \xi^{-s} \frac{d s}{s} .
$$

Moreover, let $\Lambda_{0}(f, \boldsymbol{\alpha}, \boldsymbol{\beta})$ be

$$
\begin{aligned}
& \left(\frac{q}{4 \pi^{2}}\right)^{\delta(\alpha, \beta)} \sum \sum_{a_{1}, b_{1}, a_{2}, b_{2} \geq 1} \sum_{1} \frac{\chi\left(a_{1} b_{1}\right) \mathscr{B}\left(a_{1}, b_{1} ; \boldsymbol{\alpha}\right)}{a_{1} b_{1}} \frac{\bar{\chi}\left(a_{2} b_{2}\right) \mathscr{B}\left(a_{2}, b_{2} ;-\boldsymbol{\beta}\right)}{a_{2} b_{2}} \\
& \cdot \sum_{n, m \geq 1} \sum_{\frac{\lambda_{f}}{\left(a_{1} n\right) \sigma_{3}(n ; \boldsymbol{\alpha})}} \frac{\overline{\lambda_{f}}\left(a_{2} n\right)^{1 / 2} \sigma_{3}(m ;-\boldsymbol{\beta})}{\left(a_{2} m\right)^{1 / 2}} W\left(\frac{(2 \pi)^{6} a_{1}^{3} b_{1}^{2} a_{2}^{3} b_{2}^{2} n m}{q^{3}} ; \boldsymbol{\alpha}, \boldsymbol{\beta}\right) .
\end{aligned}
$$

Lemma 3.1. We have

$$
H(0 ; \boldsymbol{\alpha}, \boldsymbol{\beta}) \Lambda(f ; \boldsymbol{\alpha}, \boldsymbol{\beta})=\Lambda_{0}(f, \boldsymbol{\alpha}, \boldsymbol{\beta})+\Lambda_{0}(f, \boldsymbol{\beta}, \boldsymbol{\alpha})
$$


Proof. We consider

$$
I:=\frac{1}{2 \pi i} \int_{(1)} \Lambda(f, s+1 / 2 ; \boldsymbol{\alpha}, \boldsymbol{\beta}) H(s ; \boldsymbol{\alpha}, \boldsymbol{\beta}) \frac{d s}{s} .
$$

Moving the contour integral to $(-1)$, we obtain that

$$
\begin{aligned}
I & =\Lambda(f ; \boldsymbol{\alpha}, \boldsymbol{\beta}) H(0 ; \boldsymbol{\alpha}, \boldsymbol{\beta})+\frac{1}{2 \pi i} \int_{(-1)} \Lambda(f, s+1 / 2 ; \boldsymbol{\alpha}, \boldsymbol{\beta}) H(s ; \boldsymbol{\alpha}, \boldsymbol{\beta}) \frac{d s}{s} \\
& =\Lambda(f ; \boldsymbol{\alpha}, \boldsymbol{\beta}) H(0 ; \boldsymbol{\alpha}, \boldsymbol{\beta})-\frac{1}{2 \pi i} \int_{(1)} \Lambda(f,-s+1 / 2 ; \boldsymbol{\alpha}, \boldsymbol{\beta}) H(-s ; \boldsymbol{\alpha}, \boldsymbol{\beta}) \frac{d s}{s} .
\end{aligned}
$$

By the functional equation, we have $\Lambda(f, s+1 / 2 ; \boldsymbol{\alpha}, \boldsymbol{\beta})=\Lambda(f,-s+1 / 2 ; \boldsymbol{\beta}, \boldsymbol{\alpha})$. Moreover, $H$ is an even function, and $H(s ; \boldsymbol{\alpha}, \boldsymbol{\beta})=H(s ; \boldsymbol{\beta}, \boldsymbol{\alpha})$. Therefore,

$$
\begin{aligned}
\Lambda(f ; \boldsymbol{\alpha}, \boldsymbol{\beta}) H(0 ; \boldsymbol{\alpha}, \boldsymbol{\beta})= & \frac{1}{2 \pi i} \int_{(1)} \Lambda(f, s+1 / 2 ; \boldsymbol{\alpha}, \boldsymbol{\beta}) H(s ; \boldsymbol{\alpha}, \boldsymbol{\beta}) \frac{d s}{s} \\
& +\frac{1}{2 \pi i} \int_{(1)} \Lambda(f, s+1 / 2 ; \boldsymbol{\beta}, \boldsymbol{\alpha}) H(s ; \boldsymbol{\alpha}, \boldsymbol{\beta}) \frac{d s}{s} .
\end{aligned}
$$

The Lemma follows after writing $\Lambda$ as a product of $L$-functions and Gamma functions and using Lemma 2.2.

Next, we let

$$
V_{\boldsymbol{\alpha}, \boldsymbol{\beta}}(\xi, \eta ; \mu)=\left(\frac{\mu}{4 \pi^{2}}\right)^{\delta(\boldsymbol{\alpha}, \boldsymbol{\beta})} \int_{-\infty}^{\infty}\left(\frac{\eta}{\xi}\right)^{i t} W\left(\frac{\xi \eta\left(4 \pi^{2}\right)^{3}}{\mu^{3}} ; \boldsymbol{\alpha}+i t, \boldsymbol{\beta}+i t\right) d t,
$$

and

$$
\begin{aligned}
\Lambda_{1}(f ; \boldsymbol{\alpha}, \boldsymbol{\beta}) & =\sum \sum_{a_{1}, b_{1}, a_{2}, b_{2} \geq 1} \sum_{a_{2}} \frac{\chi\left(a_{1} b_{1}\right) \mathscr{B}\left(a_{1}, b_{1} ; \boldsymbol{\alpha}\right)}{a_{1} b_{1}} \frac{\bar{\chi}\left(a_{2} b_{2}\right) \mathscr{B}\left(a_{2}, b_{2} ;-\boldsymbol{\beta}\right)}{a_{2}} \\
& \cdot \sum_{n, m \geq 1} \sum_{\frac{\lambda}{f}\left(a_{1} n\right) \sigma_{3}(n ; \boldsymbol{\alpha})} \frac{\overline{\lambda_{f}}\left(a_{2} m\right) \sigma_{3}(m ;-\boldsymbol{\beta})}{\left(a_{1} n\right)^{1 / 2}} V_{\boldsymbol{\alpha}, \boldsymbol{\beta}}\left(a_{1}^{3} b_{1}^{2} n, a_{2}^{3} b_{2}^{2} m ; q\right) .
\end{aligned}
$$

Lemma 3.2. With notation as above, we have

$$
H(0 ; \boldsymbol{\alpha}, \boldsymbol{\beta}) \int_{-\infty}^{\infty} \Lambda(f ; \boldsymbol{\alpha}+i t, \boldsymbol{\beta}+i t) d t=\Lambda_{1}(f ; \boldsymbol{\alpha}, \boldsymbol{\beta})+\Lambda_{1}(f ; \boldsymbol{\beta}, \boldsymbol{\alpha}) .
$$

The proof follows easily from Lemma 3.1.

Remark 1. The integration over $t$ is added so that the main contribution comes from when $a_{1}^{3} b_{1}^{2} n \ll q^{3 / 2+\varepsilon}$ and $a_{2}^{3} b_{2}^{2} m \ll q^{3 / 2+\varepsilon}$, and we will see this from Lemma 3.3 below. Without the integration over $t$, the ranges of $a_{i}, b_{j}, m, n$ that we need to consider satisfy the weaker condition $a_{1}^{3} b_{1}^{2} n a_{2}^{3} b_{2}^{2} m \ll q^{3+\varepsilon}$, and the proof presented here does not extend to this range. 
Lemma 3.3. If $\xi$ or $\eta \gg q^{\frac{3}{2}+\varepsilon}$, then for any $A>1$, we have

$$
V_{\boldsymbol{\alpha}, \boldsymbol{\beta}}(\xi, \eta ; q) \ll q^{-A},
$$

where the implied constant depends on $\varepsilon$ and $A$.

Proof. From the definition of $W$ and $V$ and a change of variables $(s+i t=w, s-i t=z)$, we can write $V_{\boldsymbol{\alpha}, \boldsymbol{\beta}}(\xi, \eta ; \mu)$ as

$$
\begin{aligned}
&\left(\frac{q}{4 \pi^{2}}\right)^{\delta(\boldsymbol{\alpha}, \boldsymbol{\beta})} \frac{4 \pi}{(2 \pi i)^{2}} \int_{(0)} \prod_{j=1}^{3} \Gamma\left(w+\frac{k-1}{2}+\alpha_{j}\right)\left(\frac{q^{\frac{3}{2}}}{\left(4 \pi^{2}\right)^{\frac{3}{2} \xi}}\right)^{w} \\
& \times \int_{(1)} \prod_{j=1}^{3} \Gamma\left(z+\frac{k-1}{2}-\beta_{j}\right)\left(\frac{q^{\frac{3}{2}}}{\left(4 \pi^{2}\right)^{\frac{3}{2}} \eta}\right)^{z} H\left(\frac{z+w}{2} ; \boldsymbol{\alpha}, \boldsymbol{\beta}\right) \frac{d z d w}{z+w} .
\end{aligned}
$$

When $\xi \gg q^{\frac{3}{2}+\varepsilon}$, we move the contour integral over $w$ to the far right, and similarly, when $\eta \gg q^{\frac{3}{2}+\varepsilon}$, we move the contour integral over $z$ to the far right. The lemma then follows.

\section{Setup For the PRoOf of 2.5 AND Diagonal TeRmS}

From Lemma 3.2, we have that for $\alpha_{i}, \beta_{i} \ll 1 / \log q$,

$$
H(0 ; \boldsymbol{\alpha}, \boldsymbol{\beta}) \mathcal{M}_{6}(q)=\frac{2}{\phi(q)} \sum_{\substack{\chi(\bmod q) \\ \chi(-1)=(-1)^{k}}} \sum_{f \in \mathcal{H}_{\chi}}^{h}\left\{\Lambda_{1}(f ; \boldsymbol{\alpha}, \boldsymbol{\beta})+\Lambda_{1}(f ; \boldsymbol{\beta}, \boldsymbol{\alpha})\right\} .
$$

Therefore, to evaluate $\mathcal{M}_{6}(q)$, it is sufficient to compute asymptotically

$$
\mathscr{M}_{1}(q ; \boldsymbol{\alpha}, \boldsymbol{\beta}):=\frac{2}{\phi(q)} \sum_{\substack{\chi(\bmod q) \\ \chi(-1)=(-1)^{k}}} \sum_{f \in \mathcal{H}_{\chi}}^{h} \Lambda_{1}(f ; \boldsymbol{\alpha}, \boldsymbol{\beta}) .
$$

Applying the Petersson's formula, we obtain that

$$
\sum_{f \in \mathcal{H}_{\chi}}^{h} \bar{\lambda}_{f}\left(a_{2} m\right) \lambda_{f}\left(a_{1} n\right)=\delta_{a_{2} m=a_{1} n}+\sigma_{\chi}\left(a_{2} m, a_{1} n\right)
$$

where $\sigma_{\chi}\left(a_{2} m, a_{1} n\right)$ is defined as in (2.3). We then write

$$
\mathscr{M}_{1}(q ; \boldsymbol{\alpha}, \boldsymbol{\beta})=\mathscr{D}(q ; \boldsymbol{\alpha}, \boldsymbol{\beta})+\mathscr{K}(q ; \boldsymbol{\alpha}, \boldsymbol{\beta}),
$$

where $\mathscr{D}(q ; \boldsymbol{\alpha}, \boldsymbol{\beta})$ is the diagonal contribution from $\delta_{a_{2} m=a_{1} n}$, and $\mathscr{K}(q ; \boldsymbol{\alpha}, \boldsymbol{\beta})$ is the contribution from $\sigma_{\chi}\left(a_{2} m, a_{1} n\right)$.

In Section 4.1 below, we will show that the term $\mathscr{D}(q ; \boldsymbol{\alpha}, \boldsymbol{\beta})$ contributes one of the twenty terms in Conjecture 2.4, specifically the term corresponding to $\mathcal{M}(q ; \boldsymbol{\alpha}+i t, \boldsymbol{\beta}+$ it). Moreover, $\mathscr{K}(q ; \boldsymbol{\alpha}, \boldsymbol{\beta})$ gives another nine terms in the conjecture, namely those transpositions in $S_{6} / S_{3} \times S_{3}$ which switches $\alpha_{i}$ and $\beta_{j}$ for a fixed $i, j=1,2,3$. We explicitly work out one of these terms in Proposition 6.1. Similarly, $\mathscr{D}(q ; \boldsymbol{\beta}, \boldsymbol{\alpha})$ gives rise to the term corresponding to $\mathcal{M}(q ; \boldsymbol{\beta}+i t, \boldsymbol{\alpha}+i t)$, and the last nine expressions arise from $\mathscr{K}(q ; \boldsymbol{\beta}, \boldsymbol{\alpha})$. 
4.1. Evaluating the diagonal terms $\mathscr{D}(q ; \boldsymbol{\alpha}, \boldsymbol{\beta})$. We recall that

$$
\begin{aligned}
& \mathscr{D}(q ; \boldsymbol{\alpha}, \boldsymbol{\beta})=\frac{2}{\phi(q)} \sum_{\substack{\chi(\bmod q) \\
\chi(-1)=(-1)^{k}}} \sum \sum_{a_{1}, b_{1}, a_{2}, b_{2}, m, n \geq 1} \sum_{a_{1} n=a_{2} m} \sum \chi\left(a_{1} b_{1}\right) \bar{\chi}\left(a_{2} b_{2}\right) \frac{\mathscr{B}\left(a_{1}, b_{1} ; \boldsymbol{\alpha}\right)}{a_{1} b_{1}} \frac{\mathscr{B}\left(a_{2}, b_{2} ;-\boldsymbol{\beta}\right)}{a_{2} b_{2}} \\
& \times \frac{\sigma_{3}(n ; \boldsymbol{\alpha}) \sigma_{3}(m ;-\boldsymbol{\beta})}{\left(a_{1} n\right)^{\frac{1}{2}}\left(a_{2} m\right)^{\frac{1}{2}}} V_{\boldsymbol{\alpha}, \boldsymbol{\beta}}\left(a_{1}^{3} b_{1}^{2} n, a_{2}^{3} b_{2}^{2} m ; q\right) .
\end{aligned}
$$

We will compute the diagonal contribution in the following lemma.

Lemma 4.1. With the same notations as above, we have

$$
\mathscr{D}(q ; \boldsymbol{\alpha}, \boldsymbol{\beta})=H(0 ; \boldsymbol{\alpha}, \boldsymbol{\beta}) \int_{-\infty}^{\infty} \mathcal{M}(q ; \boldsymbol{\alpha}+i t, \boldsymbol{\beta}+i t) d t+O\left(q^{-3 / 4+\varepsilon}\right) .
$$

Proof. We apply the orthogonality relation for Dirichlet characters in (2.10) and obtain that for $\left(a_{i} b_{i}, q\right)=1$,

$$
\frac{2}{\phi(q)} \sum_{\substack{\chi(\bmod q) \\ \chi(-1)=(-1)^{k}}} \chi\left(a_{1} b_{1}\right) \bar{\chi}\left(a_{2} b_{2}\right)= \begin{cases}1 & \text { if } a_{1} b_{1} \equiv a_{2} b_{2}(\bmod q) \\ (-1)^{k} & \text { if } a_{1} b_{1} \equiv-a_{2} b_{2}(\bmod q) \\ 0 & \text { otherwise. }\end{cases}
$$

When $a_{1} b_{1} \geq q / 4$ or $a_{2} b_{2} \geq q / 4$, we have that $a_{1}^{3} b_{1}^{2} n \geq q^{2} / 16$ or $a_{2}^{3} b_{2}^{2} m \geq q^{2} / 16$. From Lemma 3.3, $V_{\boldsymbol{\alpha}, \boldsymbol{\beta}}\left(a_{1}^{3} b_{1}^{2} n, a_{2}^{3} b_{2}^{2} m ; q\right) \ll q^{-A}$ in that range so the contribution from these terms is negligible. Hence the main contribution from $\mathscr{D}(q ; \boldsymbol{\alpha}, \boldsymbol{\beta})$ comes from the terms with $a_{1} b_{1}=a_{2} b_{2}$ when $\left(a_{i} b_{i}, q\right)=1$, and

$$
\begin{aligned}
& \mathscr{D}(q ; \boldsymbol{\alpha}, \boldsymbol{\beta})=\sum \sum_{\substack{a_{1}, b_{1}, a_{2}, b_{2}, m, n \geq 1 \\
a_{1} n=a_{2} m \\
a_{1} b_{1}=a_{2} b_{2} \\
\left(a_{i} b_{i}, q\right)=1}} \frac{\mathscr{B}\left(a_{1}, b_{1} ; \boldsymbol{\alpha}\right)}{a_{1} b_{1}} \frac{\mathscr{B}\left(a_{2}, b_{2} ;-\boldsymbol{\beta}\right)}{a_{2} b_{2}} \\
& \quad \times \frac{\sigma_{3}(n ; \boldsymbol{\alpha}) \sigma_{3}(m ;-\boldsymbol{\beta})}{\left(a_{1} n\right)^{\frac{1}{2}}\left(a_{2} m\right)^{\frac{1}{2}}} V_{\boldsymbol{\alpha}, \boldsymbol{\beta}}\left(a_{1}^{3} b_{1}^{2} n, a_{2}^{3} b_{2}^{2} m ; q\right)+O\left(q^{-A}\right) .
\end{aligned}
$$

Since $a_{1} b_{1}=a_{2} b_{2}$ and $a_{1} n=a_{2} m, a_{1}^{3} b_{1}^{2} n=a_{2}^{3} b_{2}^{2} m$. Therefore, $\mathscr{D}(q ; \boldsymbol{\alpha}, \boldsymbol{\beta})$ can be written as

$\frac{1}{2 \pi i}\left(\frac{q}{4 \pi^{2}}\right)^{\delta(\boldsymbol{\alpha}, \boldsymbol{\beta})} \int_{-\infty}^{\infty} \int_{(1)} G\left(\frac{1}{2}+s ; \boldsymbol{\alpha}+i t, \boldsymbol{\beta}+i t\right) H(s ; \boldsymbol{\alpha}, \boldsymbol{\beta})\left(\frac{q}{4 \pi^{2}}\right)^{3 s} \mathcal{A Z}\left(\frac{1}{2}+s, \boldsymbol{\alpha}, \boldsymbol{\beta}\right) \frac{d s}{s} d t$,

where we have used Equations (2.13) to (2.17).

Note that $\mathcal{A}(s ; \boldsymbol{\alpha}, \boldsymbol{\beta})$ is absolutely convergent when $\operatorname{Re}(s)>\frac{1}{4}+\varepsilon$. Furthermore, the pole at $s=-\left(\alpha_{i}-\beta_{j}\right) / 2$ from the zeta factor $\mathcal{Z}\left(\frac{1}{2}+s ; \boldsymbol{\alpha}, \boldsymbol{\beta}\right)$ is cancelled by the zero at the same point from $H(s ; \boldsymbol{\alpha}, \boldsymbol{\beta})$. Thus, in the region $\operatorname{Re}(s)>-\frac{1}{4}+\varepsilon$, the integrand is analytic except for a simple pole at $s=0$. Moving the line of integration to $\operatorname{Re}(s)=-1 / 4+\varepsilon$, we obtain that $\mathscr{D}(q ; \boldsymbol{\alpha}, \boldsymbol{\beta})$ is

$$
\left(\frac{q}{4 \pi^{2}}\right)^{\delta(\boldsymbol{\alpha}, \boldsymbol{\beta})} \int_{-\infty}^{\infty} G\left(\frac{1}{2} ; \boldsymbol{\alpha}+i t, \boldsymbol{\beta}+i t\right) H(0 ; \boldsymbol{\alpha}, \boldsymbol{\beta}) \mathcal{A Z}\left(\frac{1}{2} ; \boldsymbol{\alpha}, \boldsymbol{\beta}\right) d t+O\left(q^{-3 / 4+\varepsilon}\right) .
$$


The lemma now follows from (2.18) and upon noting that $\mathcal{A Z}\left(\frac{1}{2} ; \boldsymbol{\alpha}, \boldsymbol{\beta}\right)=\mathcal{A Z}\left(\frac{1}{2} ; \boldsymbol{\alpha}+i t, \boldsymbol{\beta}+i t\right)$.

\section{Setup For the OfF-Diagonal terms $\mathscr{K}(q ; \boldsymbol{\alpha}, \boldsymbol{\beta})$}

Define $\mathcal{K} f=i^{-k} f+i^{k} \bar{f}$. If $g$ is a real function, then $g \mathcal{K} f=\mathcal{K}(g f)$. Applying orthogonality relation for $\chi$ from $(2.10)$ to $\mathscr{K}(q ; \boldsymbol{\alpha}, \boldsymbol{\beta})$, we obtain that

$$
\begin{aligned}
& \mathscr{K}(q ; \boldsymbol{\alpha}, \boldsymbol{\beta})=2 \pi \sum_{\substack{a_{1}, b_{1}, a_{2}, b_{2} \geq 1 \\
\left(a_{1} a_{2} b_{1} b_{2}, q\right)=1}} \sum_{a_{1} b_{1}} \frac{\mathscr{B}\left(a_{1}, b_{1} ; \boldsymbol{\alpha}\right)}{a_{2} b_{2}} \sum_{n, m \geq 1} \sum_{\left.a_{2}, b_{2} ;-\boldsymbol{\beta}\right)} \frac{\sigma_{3}(n ; \boldsymbol{\alpha})}{\left(a_{1} n\right)^{1 / 2}} \frac{\sigma_{3}(m ;-\boldsymbol{\beta})}{\left(a_{2} m\right)^{1 / 2}} \\
& \quad \times V_{\boldsymbol{\alpha}, \boldsymbol{\beta}}\left(a_{1}^{3} b_{1}^{2} n, a_{2}^{3} b_{2}^{2} m ; q\right) \sum_{c=1}^{\infty} \frac{1}{c q} J_{k-1}\left(\frac{4 \pi}{c q} \sqrt{a_{2} m a_{1} n}\right) \mathcal{K} \sum_{\substack{a \\
a\left(\bmod b_{1} a_{2} b_{2}(\bmod q)\right.}}^{*} e\left(\frac{a a_{2} m+\bar{a} a_{1} n}{c q}\right),
\end{aligned}
$$

where $\sum^{*}$ denotes a sum over reduced residues. Let $f$ be a smooth partition of unity such that

$$
\sum_{M}^{d} f\left(\frac{m}{M}\right)=1
$$

where $f$ is supported in $[1 / 2,3]$ and $\sum_{M}^{d}$ denotes an dyadic sum over $M=2^{k}, k \geq 0$.

Rearranging the sum, we have

$$
\mathscr{K}(q ; \boldsymbol{\alpha}, \boldsymbol{\beta})=\frac{2 \pi}{q} \sum_{\substack{a_{1}, b_{1}, a_{2}, b_{2} \geq 1 \\\left(a_{1} a_{2} b_{1} b_{2}, q\right)=1}} \sum_{a_{1}^{2} b_{1}} \frac{\mathscr{B}\left(a_{1}, b_{1} ; \boldsymbol{\alpha}\right)}{a_{2}^{\frac{3}{2}}\left(a_{2}, b_{2} ;-\boldsymbol{\beta}\right)} \sum_{M}^{d} \sum_{N}^{d} b_{2} S(\mathbf{a}, \mathbf{b}, M, N ; \boldsymbol{\alpha}, \boldsymbol{\beta}),
$$

where

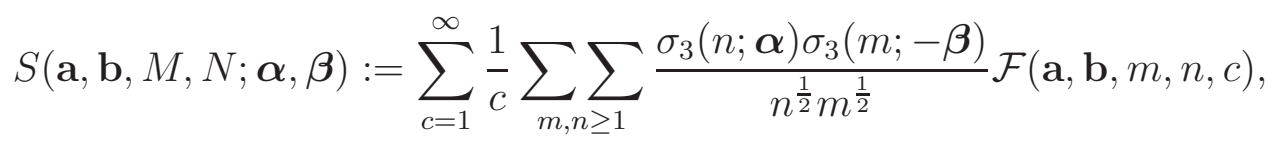

$$
\mathcal{F}(\mathbf{a}, \mathbf{b}, m, n, c):=\mathcal{G}(\mathbf{a}, \mathbf{b}, m, n, c) \mathcal{K} \sum_{\substack{a \equiv(\bmod c q) \\ a a_{1} b_{1} a_{2} b_{2}(\bmod q)}}^{*} e\left(\frac{a a_{2} m+\bar{a} a_{1} n}{c q}\right) J_{k-1}\left(\frac{4 \pi}{c q} \sqrt{a_{2} m a_{1} n}\right),
$$

and

$$
\mathcal{G}(\mathbf{a}, \mathbf{b}, m, n, c):=V_{\boldsymbol{\alpha}, \boldsymbol{\beta}}\left(a_{1}^{3} b_{1}^{2} n, a_{2}^{3} b_{2}^{2} m ; q\right) f\left(\frac{m}{M}\right) f\left(\frac{n}{N}\right) .
$$

As described in the outline of the paper, we now take the following steps to compute $\mathscr{K}(q ; \boldsymbol{\alpha}, \boldsymbol{\beta})$.

We write

$$
\mathscr{K}(q ; \boldsymbol{\alpha}, \boldsymbol{\beta})=\mathscr{K}_{M}(q ; \boldsymbol{\alpha}, \boldsymbol{\beta})+\mathscr{K}_{E}(q ; \boldsymbol{\alpha}, \boldsymbol{\beta})
$$


where $\mathscr{K}_{M}(q ; \boldsymbol{\alpha}, \boldsymbol{\beta})$ is the contribution from the sum over $c<C$, where $C=\frac{\sqrt{a_{1} a_{2} M N}}{q^{\frac{2}{3}}}$, and $\mathscr{K}_{E}(q ; \boldsymbol{\alpha}, \boldsymbol{\beta})$ is the rest. We will show that the contribution from $\mathscr{K}_{E}(q ; \boldsymbol{\alpha}, \boldsymbol{\beta})$ is small in Section 5.1. This is possible by the decay of the Bessel functions and such a truncation bounds the size of the conductor inside the exponential sum.

For $\mathscr{K}_{M}(q ; \boldsymbol{\alpha}, \boldsymbol{\beta})$, we start by reducing the conductor inside the exponential sum from $c q$ to $c$ in Section 5.2. This step takes advantage of the average over $\chi \bmod q$.

Before we show each step, we provide properties of Bessel functions that will be used later.

Lemma 5.1. We have

$$
J_{k-1}(2 \pi x)=\frac{1}{\pi \sqrt{x}}\left\{W(2 \pi x) \mathrm{e}\left(x-\frac{k}{4}+\frac{1}{8}\right)+\bar{W}(2 \pi x) \mathrm{e}\left(-x+\frac{k}{4}-\frac{1}{8}\right)\right\},
$$

where $W^{(j)}(x) \ll_{j, k} x^{-j}$. Moreover,

$$
J_{k-1}(2 x)=\sum_{\ell=0}^{\infty}(-1)^{\ell} \frac{x^{2 \ell+k-1}}{\ell !(\ell+k-1) !},
$$

and

$$
J_{k-1}(x) \ll \min \left(x^{-1 / 2}, x^{k-1}\right) .
$$

Finally, the following integration is used when calculating the main terms of $\mathscr{K}(q ; \boldsymbol{\alpha}, \boldsymbol{\beta})$. If $\alpha, \beta, \gamma>0$, then

$$
\mathcal{K} \int_{0}^{\infty} \mathrm{e}\left((\alpha+\beta) x+\gamma x^{-1}\right) J_{k-1}(4 \pi \sqrt{\alpha \beta x}) \frac{d x}{x}=2 \pi J_{k-1}(4 \pi \sqrt{\alpha \gamma}) J_{k-1}(4 \pi \sqrt{\beta \gamma})
$$

and the integration is 0 if $\alpha, \beta>0$ and $\gamma \leq 0$.

These results are standard. We refer the reader to [22] for the first three claims, and to [18] for the last claim.

5.1. Truncating the sum over $c$. In this section we show that we can truncate the sum over $c$ in $S(\mathbf{a}, \mathbf{b}, M, N ; \boldsymbol{\alpha}, \boldsymbol{\beta})$ with small error contribution.

Proposition 5.2. Let $C=\frac{\sqrt{a_{1} a_{2} M N}}{q^{\frac{2}{3}}}, k \geq 5$, and $\mathcal{F}(\mathbf{a}, \mathbf{b}, m, n, c)$ be defined as in (5.1).

Further, let

$$
\mathscr{K}_{E}(q ; \boldsymbol{\alpha}, \boldsymbol{\beta})=\frac{2 \pi}{q} \sum_{\substack{a_{1}, b_{1}, a_{2}, b_{2} \geq 1 \\\left(a_{1} a_{2} b_{1} b_{2}, q\right)=1}} \frac{\mathscr{B}\left(a_{1}, b_{1} ; \boldsymbol{\alpha}\right)}{a_{1}^{\frac{3}{2}} b_{1}} \frac{\mathscr{B}\left(a_{2}, b_{2} ;-\boldsymbol{\beta}\right)}{a_{2}^{\frac{3}{2}} b_{2}} \sum_{M}^{d} \sum_{N}^{d} S_{E}(\mathbf{a}, \mathbf{b}, M, N ; \boldsymbol{\alpha}, \boldsymbol{\beta}),
$$

where

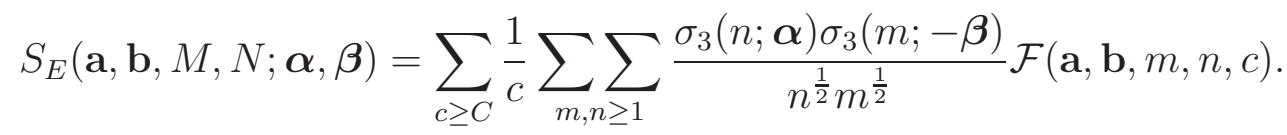

Then

$$
\mathscr{K}_{E}(q ; \boldsymbol{\alpha}, \boldsymbol{\beta}) \ll q^{-\frac{5}{12}+\varepsilon}
$$


Proof. Note that the contribution of terms when $a_{1}^{3} b_{1}^{2} N$ or $a_{2}^{3} b_{2}^{2} M$ is $\gg q^{\frac{3}{2}+\varepsilon}$ is $\ll_{\varepsilon, A} q^{-A}$, due to the fast decay rate of $\mathcal{G}(\mathbf{a}, \mathbf{b}, m, n, c)$ defined in (5.2). Thus we will discount such terms in the rest of the proof. For $k \geq 5$, we let

$$
\begin{aligned}
\tilde{\Delta}(m, n) & =\tilde{\Delta}(m, n, \mathbf{a}, \mathbf{b} ; C) \\
& =\sum_{c \geq C} \frac{1}{c} \mathcal{K} \sum_{\substack{a \equiv a_{1} b_{1} a_{2} b_{2}(\bmod q) \\
c q}}^{*} e\left(\frac{a a_{2} m+\bar{a} a_{1} n}{c q}\right) J\left(\frac{4 \pi \sqrt{a_{1} a_{2} m n}}{c q}\right) \\
& =\tilde{\Delta}_{1}(m, n)+\tilde{\Delta}_{2}(m, n),
\end{aligned}
$$

where

$$
\tilde{\Delta}_{1}(m, n):=\sum_{\substack{c \geq C \\(c, q)=1}} \frac{1}{c} \mathcal{K} \sum_{\substack{a \equiv \overline{a_{1} b_{1}} a_{2} b_{2}(\bmod q) \\ a q}}^{*} e\left(\frac{a a_{2} m+\bar{a} a_{1} n}{c q}\right) J\left(\frac{4 \pi \sqrt{a_{1} a_{2} m n}}{c q}\right),
$$

and $\tilde{\Delta}_{2}(m, n)$ is the sum of the terms where $(c, q)>1$. Now for $(c, q)=1$, the Weil bound gives

$$
\sum_{a \equiv \overline{a(\bmod c q)}}^{*} e\left(\frac{a a_{2} m+\bar{a} a_{1} n}{c q}\right) \ll c^{1 / 2+\varepsilon} \sqrt{\left(a_{1} m, a_{2} n, c\right)},
$$

and from the bound in (5.6), we have

$$
J\left(\frac{4 \pi \sqrt{a_{1} a_{2} m n}}{c q}\right) \ll\left(\frac{\sqrt{a_{1} a_{2} m n}}{c q}\right)^{k-1} .
$$

When $a_{1}^{3} b_{1}^{2} N$ and $a_{2}^{3} b_{2}^{2} M \ll q^{\frac{3}{2}+\varepsilon}$, we obtain that for $k \geq 5$,

$$
\sum_{m, n} \frac{\sigma_{3}(n ; \boldsymbol{\alpha}) \sigma_{3}(m ;-\boldsymbol{\beta})}{\sqrt{m n}} \tilde{\Delta}_{1}(m, n) \mathcal{G}(\mathbf{a}, \mathbf{b}, m, n, c) \ll q^{\varepsilon} M^{\frac{k}{2}} N^{\frac{k}{2}} \sum_{c \geq C} \frac{1}{c} c^{1 / 2+\varepsilon}\left(\frac{\sqrt{a_{1} a_{2}}}{c q}\right)^{k-1} \ll q^{\frac{7}{12}+\varepsilon} .
$$

In the above, we have used that $\max \left(a_{1}^{3} b_{1}^{2} N, a_{2}^{3} b_{2}^{2} M\right) \ll q^{\frac{3}{2}+\varepsilon}$. Then summing over $a_{1}, b_{1}, a_{2}, b_{2}$ gives the desired bound.

Now, for $(c, q)>1$, we use the bound

$$
\sum_{a \equiv \frac{a(\bmod c q)}{a b_{1} b_{1} a_{2} b_{2}(\bmod q)}}^{*} e\left(\frac{a a_{2} m+\bar{a} a_{1} n}{c q}\right) \ll(c q)^{1 / 2+\varepsilon} \sqrt{\left(a_{1} m, a_{2} n, c q\right)} .
$$

Hence, for $q \mid c$ and $k \geq 5$, we obtain

$$
\sum_{m, n} \frac{\sigma_{3}(n ; \boldsymbol{\alpha}) \sigma_{3}(m ;-\boldsymbol{\beta})}{\sqrt{m n}} \tilde{\Delta}_{2}(m, n) \mathcal{G}(\mathbf{a}, \mathbf{b}, m, n, c) \ll q^{\varepsilon} M^{\frac{k}{2}} N^{\frac{k}{2}} \sum_{\substack{c \geq C \\ q \mid c}} \frac{1}{c}(q c)^{\frac{1}{2}}\left(\frac{\sqrt{a_{1} a_{2}}}{c q}\right)^{k-1} \ll q^{\frac{1}{12}+\varepsilon} .
$$

Then summing over $a_{1}, b_{1}, a_{2}, b_{2}$ gives the desired bound. 
From this proposition, we are left to consider only

$\mathscr{K}_{M}(q ; \boldsymbol{\alpha}, \boldsymbol{\beta})=\frac{2 \pi}{q} \sum_{\substack{a_{1}, b_{1}, a_{2}, b_{2} \geq 1 \\\left(a_{1} a_{2} b_{1} b_{2}, q\right)=1}} \sum_{a_{1}} \frac{\mathscr{B}\left(a_{1}, b_{1} ; \boldsymbol{\alpha}\right)}{a_{1}^{\frac{3}{2}} b_{1}} \frac{\mathscr{B}\left(a_{2}, b_{2} ;-\boldsymbol{\beta}\right)}{a_{2}^{\frac{3}{2}} b_{2}} \sum_{M}^{d} \sum_{N}^{d} S_{M}(\mathbf{a}, \mathbf{b}, M, N ; \boldsymbol{\alpha}, \boldsymbol{\beta})$,

where

$$
S_{M}(\mathbf{a}, \mathbf{b}, M, N ; \boldsymbol{\alpha}, \boldsymbol{\beta}):=\sum_{c<C} \frac{1}{c} \sum_{m, n \geq 1} \frac{\sigma_{3}(n ; \boldsymbol{\alpha}) \sigma_{3}(m ;-\boldsymbol{\beta})}{n^{\frac{1}{2}} m^{\frac{1}{2}}} \mathcal{F}(\mathbf{a}, \mathbf{b}, m, n, c) .
$$

5.2. Treatment of the exponential sum. Next, we reduce the conductor in the exponential sum in $\mathcal{F}(\mathbf{a}, \mathbf{b}, m, n, c)$ before applying Voronoi summation.

Lemma 5.3 (Treatment of the exponential sum). Assume that $(c, q)=1$ and let

$$
Y:=\sum_{\substack{a(\bmod c q) \\ a \equiv \frac{a_{1} b_{1}}{a_{2} b_{2}}(\bmod q)}}^{*} e\left(\frac{a u+\bar{a} v}{c q}\right) .
$$

Then we have

$$
Y=e\left(\frac{\left(a_{2} b_{2}\right)^{2} u+\left(a_{1} b_{1}\right)^{2} v}{c q a_{1} b_{1} a_{2} b_{2}}\right) \sum_{x(\bmod c)}^{*} e\left(\frac{\bar{q}\left(a_{1} b_{1} x-a_{2} b_{2}\right) u}{a_{1} b_{1} c}\right) e\left(\frac{\bar{q}\left(a_{2} b_{2} \bar{x}-a_{1} b_{1}\right) v}{a_{2} b_{2} c}\right) .
$$

Proof. By Chinese Remainder Theorem, for each $a(\bmod c q)$, there exist unique $x(\bmod c)$ and $y(\bmod q)$ such that

$$
a=x q \bar{q}+y c \bar{c}
$$

where $\bar{q}$ denotes the inverse of $q$ modulo $c$, and $\bar{c}$ denotes the inverse of $c$ modulo $q$. Using (5.10) and the reciprocity relation

$$
\frac{\bar{a}}{b}+\frac{\bar{b}}{a} \equiv \frac{1}{a b}(\bmod 1)
$$

where $(a, b)=1, \bar{a}$ is the inverse of $a \bmod b$, and $\bar{b}$ is the inverse of $b \bmod a$, we obtain that

$$
Y=e\left(\frac{\overline{a_{1} b_{1}} a_{2} b_{2} \bar{c} u+a_{1} b_{1} \overline{a_{2} b_{2}} \bar{c} v}{q}\right) \sum_{x(\bmod c)}^{*} e\left(\frac{x \bar{q} u+\bar{x} \bar{q} v}{c}\right)
$$

Thus

$$
Y=e\left(\frac{\left(a_{2} b_{2}\right)^{2} u+\left(a_{1} b_{1}\right)^{2} v}{c q a_{1} b_{1} a_{2} b_{2}}\right) \sum_{x(\bmod c)}^{*} e\left(\frac{x \bar{q} u+\bar{x} \bar{q} v}{c}\right) e\left(\frac{-\bar{q} a_{2} b_{2} u}{c a_{1} b_{1}}\right) e\left(\frac{-\bar{q} a_{1} b_{1} v}{c a_{2} b_{2}}\right),
$$

and the lemma follows. 
Note that when $c<C<q$, we automatically have $(c, q)=1$. The point of this lemma is that we may treat $e\left(\frac{\left(a_{2} b_{2}\right)^{2} u+\left(a_{1} b_{1}\right)^{2} v}{c q a_{1} b_{1} a_{2} b_{2}}\right)$ as a smooth function with small derivatives, while the other exponentials have conductor at most $c a_{i} b_{i} \leq q^{1+\varepsilon}$ after truncation. It should be noted however, that we are most concerned with the contribution from the transition region of the Bessel function, where the conductor $c a_{i} b_{i}$ should be thought of as around size $q^{1 / 2}$.

\section{Applying Voronoi Summation}

To calculate $\mathscr{K}_{M}(q ; \boldsymbol{\alpha}, \boldsymbol{\beta})$ as defined in (5.8), we start by evaluating $S_{M}(\mathbf{a}, \mathbf{b}, M, N ; \boldsymbol{\alpha}, \boldsymbol{\beta})$ defined in (5.9). We write

$$
S_{M}(\mathbf{a}, \mathbf{b}, M, N ; \boldsymbol{\alpha}, \boldsymbol{\beta})=\sum_{c<C} \sum_{(\bmod c)}^{*} \frac{1}{c}\left(\mathcal{S}_{\boldsymbol{\alpha}, \boldsymbol{\beta}}^{+}(c, x)+\mathcal{S}_{\boldsymbol{\alpha}, \boldsymbol{\beta}}^{-}(c, x)\right),
$$

where

$$
\begin{aligned}
\mathcal{S}_{\boldsymbol{\alpha}, \boldsymbol{\beta}}^{ \pm}(c, x)= & i^{\mp k} \sum_{m \geq 1} \frac{\sigma_{3}(m ;-\boldsymbol{\beta})}{m^{\frac{1}{2}}} f\left(\frac{m}{M}\right) e\left( \pm \frac{a_{2}^{2} b_{2} m}{c q a_{1} b_{1}}\right) e\left( \pm \frac{\bar{q}\left(a_{1} b_{1} x-a_{2} b_{2}\right) a_{2} m}{a_{1} b_{1} c}\right) \times \\
& \times \sum_{n \geq 1} \sigma_{3}(n ; \boldsymbol{\alpha}) F_{\boldsymbol{\alpha}, \boldsymbol{\beta}}^{ \pm}(m, n, c) e\left( \pm \frac{\bar{q}\left(a_{2} b_{2} \bar{x}-a_{1} b_{1}\right) a_{1} n}{a_{2} b_{2} c}\right),
\end{aligned}
$$

where

$$
F_{\boldsymbol{\alpha}, \boldsymbol{\beta}}^{ \pm}(m, n, c)=\frac{1}{n^{\frac{1}{2}}} V_{\boldsymbol{\alpha}, \boldsymbol{\beta}}\left(a_{1}^{3} b_{1}^{2} n, a_{2}^{3} b_{2}^{2} m ; q\right) J_{k-1}\left(\frac{4 \pi}{c q} \sqrt{a_{2} m a_{1} n}\right) e\left( \pm \frac{a_{1}^{2} b_{1} n}{c q a_{2} b_{2}}\right) f\left(\frac{n}{N}\right) .
$$

Let

$$
\frac{\lambda_{1}}{\eta_{1}}=\frac{\bar{q}\left(a_{2} b_{2} \bar{x}-a_{1} b_{1}\right) a_{1}}{a_{2} b_{2} c}
$$

and

$$
\frac{\lambda_{2}}{\eta_{2}}=\frac{\bar{q}\left(a_{1} b_{1} x-a_{2} b_{2}\right) a_{2}}{a_{1} b_{1} c},
$$

where $\left(\lambda_{1}, \eta_{1}\right)=\left(\lambda_{2}, \eta_{2}\right)=1$. Moreover we define

$$
\begin{aligned}
& \mathcal{V}_{\boldsymbol{\alpha}, \boldsymbol{\beta}}^{ \pm}(c ; \mathbf{a}, \mathbf{b}, y, z)=\frac{1}{y^{1 / 2}} \frac{1}{z^{1 / 2}} V_{\boldsymbol{\alpha}, \boldsymbol{\beta}}\left(a_{1}^{3} b_{1}^{2} y, a_{2}^{3} b_{2}^{2} z ; q\right) J_{k-1}\left(\frac{4 \pi \sqrt{a_{2} z a_{1} y}}{c q}\right) \times \\
& \times i^{\mp k} \mathrm{e}\left( \pm \frac{a_{1}^{2} b_{1} y}{c q a_{2} b_{2}} \pm \frac{a_{2}^{2} b_{2} z}{c q a_{1} b_{1}}\right) f\left(\frac{y}{N}\right) f\left(\frac{z}{M}\right) .
\end{aligned}
$$

We then apply Voronoi Summation as in Theorem B.1 to the sum over $n, m$ and obtain that $\mathcal{S}_{\boldsymbol{\alpha}, \boldsymbol{\beta}}^{+}(c, x)+\mathcal{S}_{\boldsymbol{\alpha}, \boldsymbol{\beta}}^{-}(c, x)$ is

$$
\sum_{i=1}^{3} \sum_{j=1}^{3} \operatorname{Res}_{s_{1}=1-\alpha_{i}} \operatorname{Res}_{s_{2}=1+\beta_{j}}\left(\mathcal{T}_{M, \boldsymbol{\alpha}, \boldsymbol{\beta}}^{+}\left(c, x, s_{1}, s_{2}\right)+\mathcal{T}_{M, \boldsymbol{\alpha}, \boldsymbol{\beta}}^{-}\left(c, x, s_{1}, s_{2}\right)\right)+\sum_{i=1}^{8}\left(\mathcal{T}_{i, \boldsymbol{\alpha}, \boldsymbol{\beta}}^{+}(c, x)+\mathcal{T}_{i, \boldsymbol{\alpha}, \boldsymbol{\beta}}^{-}(c, x)\right),
$$


where in the region of absolutely convergence,

$$
\begin{gathered}
\mathcal{T}_{M, \boldsymbol{\alpha}, \boldsymbol{\beta}}^{ \pm}\left(c, x, s_{1}, s_{2}\right):=\mathcal{F}_{M}^{ \pm}(c ; \boldsymbol{\alpha}, \boldsymbol{\beta}) D_{3}\left(s_{1}, \pm \frac{\lambda_{1}}{\eta_{1}}, \boldsymbol{\alpha}\right) D_{3}\left(s_{2}, \pm \frac{\lambda_{2}}{\eta_{2}},-\boldsymbol{\beta}\right) \\
\mathcal{F}_{M}^{ \pm}(c ; \boldsymbol{\alpha}, \boldsymbol{\beta}):=\mathcal{F}_{M}^{ \pm}\left(c, s_{1}, s_{2} ; \boldsymbol{\alpha}, \boldsymbol{\beta}\right):=\int_{0}^{\infty} \int_{0}^{\infty} y^{s_{1}-1} z^{s_{2}-1} \mathcal{V}_{\boldsymbol{\alpha}, \boldsymbol{\beta}}^{ \pm}(c ; \mathbf{a}, \mathbf{b}, y, z) d y d z,
\end{gathered}
$$

$$
\begin{aligned}
& \mathcal{T}_{1, \boldsymbol{\alpha}, \boldsymbol{\beta}}^{ \pm}(c, x):=\frac{\pi^{3 / 2+\alpha_{1}+\alpha_{2}+\alpha_{3}}}{\eta_{1}^{3+\alpha_{1}+\alpha_{2}+\alpha_{3}}} \sum_{i=1}^{3} \operatorname{Res}_{s=1+\beta_{i}} D_{3}\left(s_{2}, \pm \frac{\lambda_{2}}{\eta_{2}},-\boldsymbol{\beta}\right) \sum_{n=1}^{\infty} A_{3}\left(n, \pm \frac{\lambda_{1}}{\eta_{1}}, \boldsymbol{\alpha}\right) \mathcal{F}_{1}^{ \pm}(c, n ; \boldsymbol{\alpha}, \boldsymbol{\beta}), \\
& \mathcal{F}_{1}^{ \pm}(c, n ; \boldsymbol{\alpha}, \boldsymbol{\beta})=\mathcal{F}_{1}^{ \pm}(c, n, s ; \boldsymbol{\alpha}, \boldsymbol{\beta})=\int_{0}^{\infty} \int_{0}^{\infty} z^{s-1} \mathcal{V}_{\boldsymbol{\alpha}, \boldsymbol{\beta}}^{ \pm}(c ; \mathbf{a}, \mathbf{b}, y, z) U_{3}\left(\frac{\pi^{3} n y}{\eta_{1}^{3}} ; \boldsymbol{\alpha}\right) d y d z,
\end{aligned}
$$

and $\mathcal{T}_{i, \boldsymbol{\alpha}, \boldsymbol{\beta}}^{ \pm}(c, x)$, where $i=2,3,4$ are defined similarly. Further,

$$
\begin{gathered}
\mathcal{T}_{5, \boldsymbol{\alpha}, \boldsymbol{\beta}}^{ \pm}(c, x):=\frac{\pi^{3+\sum_{i=1}^{3}\left(\alpha_{i}-\beta_{i}\right)}}{\eta_{1}^{3+\sum_{i=1}^{3} \alpha_{i}} \eta_{2}^{3-\sum_{i=1}^{3} \beta_{i}}} \sum_{n=1}^{\infty} \sum_{m=1}^{\infty} A_{3}\left(n, \pm \frac{\lambda_{1}}{\eta_{1}}, \boldsymbol{\alpha}\right) A_{3}\left(m, \pm \frac{\lambda_{2}}{\eta_{2}},-\boldsymbol{\beta}\right) \mathcal{F}_{5}^{ \pm}(c, n, m ; \boldsymbol{\alpha}, \boldsymbol{\beta}), \\
\mathcal{F}_{5}^{ \pm}(c, n, m ; \boldsymbol{\alpha}, \boldsymbol{\beta})=\int_{0}^{\infty} \int_{0}^{\infty} \mathcal{V}_{\boldsymbol{\alpha}, \boldsymbol{\beta}}^{ \pm}(c ; \mathbf{a}, \mathbf{b}, y, z) U_{3}\left(\frac{\pi^{3} m z}{\eta_{2}^{3}} ;-\boldsymbol{\beta}\right) U_{3}\left(\frac{\pi^{3} n y}{\eta_{1}^{3}} ; \boldsymbol{\alpha}\right) d y d z
\end{gathered}
$$

and $\mathcal{T}_{i, \boldsymbol{\alpha}, \boldsymbol{\beta}}^{ \pm}(c, x)$, where $i=6,7,8$ are defined similarly.

As mentioned in Section 4 , there are nine terms from $\mathscr{K}(q ; \boldsymbol{\alpha}, \boldsymbol{\beta})$. In particular, we will show that these terms arise from

$$
\sum_{c<C} \sum_{(\bmod c)}^{*} \frac{1}{c}\left(\mathcal{T}_{M, \boldsymbol{\alpha}, \boldsymbol{\beta}}^{+}\left(c, x, s_{1}, s_{2}\right)+\mathcal{T}_{M, \boldsymbol{\alpha}, \boldsymbol{\beta}}^{-}\left(c, x, s_{1}, s_{2}\right)\right),
$$

and in fact each term comes from the residues at $s_{1}=1-\alpha_{i}$ and $s_{2}=1+\beta_{j}$ for $i=1,2,3$. We state the contribution from the residues $s_{1}=1-\alpha_{1}$ and $s_{2}=1+\beta_{1}$ in Proposition 6.1 below, and prove it in Section 7. By symmetry, the analogous result holds for the other residues. Then, we will show that the rest of $\mathcal{T}_{i, \boldsymbol{\alpha}, \boldsymbol{\beta}}^{ \pm}(c, x)$ are negligible in Section 8 as stated in Proposition 6.2.

Proposition 6.1. Let

$$
\begin{aligned}
R_{\alpha_{1}, \beta_{1}}:=\frac{2 \pi}{q} \sum_{\substack{a_{1}, b_{1}, a_{2}, b_{2} \geq 1 \\
\left(a_{1} a_{2} b_{1} b_{2}, q\right)=1}} \frac{\mathscr{B}\left(a_{1}, b_{1} ; \boldsymbol{\alpha}\right)}{a_{1}^{\frac{3}{2}} b_{1}} \frac{\mathscr{B}\left(a_{2}, b_{2} ;-\boldsymbol{\beta}\right)}{a_{2}^{\frac{3}{2}} b_{2}} \\
\cdot \sum_{M}^{d} \sum_{N} \sum_{c<C} \sum_{x}^{*} \frac{1}{(\bmod c)} \underset{s_{1}=1-\alpha_{1}}{\operatorname{Res}} \underset{s_{2}=1+\beta_{1}}{\operatorname{Res}}\left(\mathcal{T}_{M, \boldsymbol{\alpha}, \boldsymbol{\beta}}^{+}\left(c, x, s_{1}, s_{2}\right)+\mathcal{T}_{M, \boldsymbol{\alpha}, \boldsymbol{\beta}}^{-}\left(c, x, s_{1}, s_{2}\right)\right) .
\end{aligned}
$$


Then we have

$$
R_{\alpha_{1}, \beta_{1}}=H(0 ; \boldsymbol{\alpha}, \boldsymbol{\beta}) \int_{-\infty}^{\infty} \mathcal{M}(q ; \pi(\boldsymbol{\alpha})+i t, \pi(\boldsymbol{\beta})+i t) d t+O\left(q^{-1 / 2+\varepsilon}\right),
$$

where $(\pi(\boldsymbol{\alpha}), \pi(\boldsymbol{\beta}))=\left(\beta_{1}, \alpha_{2}, \alpha_{3} ; \alpha_{1}, \beta_{2}, \beta_{3}\right)$.

Proposition 6.2. For $i=1, \ldots, 8$, define

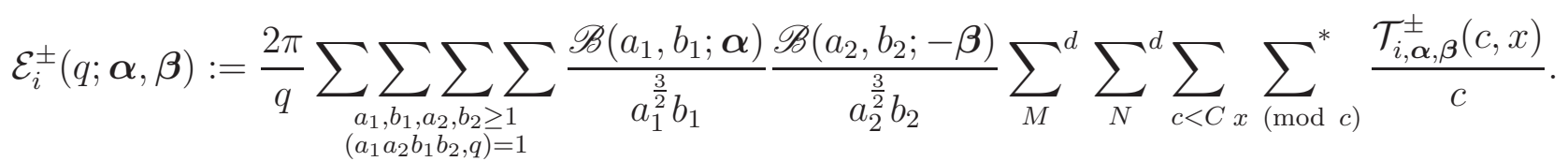

Then

$$
\mathcal{E}_{i}^{ \pm}(q ; \boldsymbol{\alpha}, \boldsymbol{\beta}) \ll q^{-1 / 4+\varepsilon}
$$

We will prove this proposition in Section 8.

\section{Proof of Proposition 6.1}

We begin by collecting some lemmas which will be used in this section.

\subsection{Preliminary Lemmas.}

Lemma 7.1. Let $(a, \ell)=1$. We have

$$
f(c, \ell):=\sum_{\substack{x(\bmod c) \\ x \equiv a(\bmod \ell)}}^{*} 1=c \prod_{\substack{p \mid c \\ p \nmid \ell}}\left(1-\frac{1}{p}\right)=\phi(c) \prod_{p \mid(\ell, c)}\left(1-\frac{1}{p}\right)^{-1} .
$$

Proof. We first prove that if $(m, n \ell)=1$, then

$$
f(m n, \ell)=f(n, \ell) \phi(m) .
$$

For all $x$ satisfying $(x, m n \ell)=1$, we can write $x=u m \bar{m}+v n \ell \overline{n \ell}$, where $m \bar{m} \equiv$ $1(\bmod n \ell), n \ell \overline{n \ell} \equiv 1(\bmod m)$, and $(u, n \ell)=(v, m)=1$. Moreover $x \equiv a(\bmod \ell)$ if and only if $u \equiv a(\bmod \ell)$. By Chinese Remainder Theorem,

$$
f(m n, \ell)=\sum_{\substack{x(\bmod m n \ell) \\ x \equiv a(\bmod \ell)}}^{*} 1=\sum_{\substack{u(\bmod n \ell) \\ u \equiv a(\bmod \ell)}}^{*} \sum_{(\bmod m)}^{*} 1=f(n, \ell) \phi(m) .
$$

Let $c=c_{1} c_{2}$, where all prime factors of $c_{1}$ also divide $\ell$, and $\left(c_{2}, \ell\right)=1$. From $(7.1)$, we have that $f(c, \ell)=f\left(c_{1}, \ell\right) \phi\left(c_{2}\right)$.

Now let $x$ be any residue modulo $c_{1} \ell$ with $x \equiv a(\bmod \ell)$. Then $\left(x, c_{1} \ell\right)=1$ since $(a, \ell)=1$. Thus all such $x$ can be uniquely written as $x=a+k \ell$, where $k=0, \ldots, c_{1}-1$, so $f\left(c_{1}, \ell\right)=c_{1}$. We then have $f(c, \ell)=c_{1} \phi\left(c_{2}\right)$, and the statement follows from the identity $\phi\left(c_{2}\right)=c_{2} \prod_{p \mid c_{2}}\left(1-\frac{1}{p}\right)$. 
Lemma 7.2. Let $\alpha, \beta, y, z$ be nonnegative real numbers satisfying $\alpha y, \beta z \ll q^{2}$ and define

$$
T=T(y, z, \alpha, \beta):=\sum_{\delta=1}^{\infty} \frac{1}{\delta} J_{k-1}\left(\frac{4 \pi \sqrt{\alpha \beta y z}}{\delta}\right) \mathcal{K} \mathrm{e}\left(\frac{\alpha y}{\delta}+\frac{\beta z}{\delta}\right) .
$$

Further, let $L=q^{100}$ and $w$ be a smooth function on $\mathbb{R}^{+}$with $w(x)=1$ if $0 \leq x \leq 1$, and $w(x)=0$ if $x>2$. Then for any $A>0$, we have

$$
\begin{aligned}
T= & 2 \pi \sum_{\ell=1}^{\infty} w\left(\frac{\ell}{L}\right) J_{k-1}(4 \pi \sqrt{\alpha y \ell}) J_{k-1}(4 \pi \sqrt{\beta z \ell}) \\
& -2 \pi \int_{0}^{\infty} w\left(\frac{\ell}{L}\right) J_{k-1}(4 \pi \sqrt{\alpha y \ell}) J_{k-1}(4 \pi \sqrt{\beta z \ell}) d \ell+O_{A}\left(q^{-A}\right) .
\end{aligned}
$$

Proof. We will follow Iwaniec and Xiaoqing Li's arguments in Section 3 of [13] to evaluate $T$. Let $\eta(s)$ be a smooth function on $\mathbb{R}^{+}$with $\eta(s)=0$ if $0 \leq s<1 / 4,0 \leq \eta(s) \leq 1$ if $1 / 4 \leq s \leq 1 / 2$, and $\eta(s)=1$ if $s>1 / 2$. We then obtain that

$$
T=\mathcal{K} \sum_{\delta} \frac{\eta(\delta)}{\delta} J_{k-1}\left(\frac{4 \pi \sqrt{\alpha \beta y z}}{\delta}\right) \mathrm{e}\left(\frac{\alpha y}{\delta}+\frac{\beta z}{\delta}\right) .
$$

After inserting this smooth function we apply Poisson summation to obtain that

$$
T=\sum_{\ell} \hat{F}(\ell):=\sum_{\ell} \mathcal{K} \int_{0}^{\infty} \frac{\eta(u)}{u} \mathrm{e}\left(\ell u+\frac{\alpha y}{u}+\frac{\beta z}{u}\right) J_{k-1}\left(\frac{4 \pi \sqrt{\alpha \beta y z}}{u}\right) d u .
$$

By (5.4), we can write the integral above in terms of two integrals with the phase

$$
\ell u+\frac{\alpha y \pm \sqrt{\alpha \beta y z}+\beta z}{u} .
$$

If $|\ell|>L$, the factor $\ell u$ dominates. Then integrating by parts $A$ times, we have that

$$
\int_{0}^{\infty} \frac{\eta(u)}{u} \mathrm{e}\left(\ell u+\frac{\alpha y}{u}+\frac{\beta z}{u}\right) J_{k-1}\left(\frac{4 \pi \sqrt{\alpha \beta y z}}{u}\right) d u \ll q^{-A} .
$$

Therefore

$$
T=\sum_{\ell} \hat{F}(\ell) w\left(\frac{|\ell|}{L}\right)+O\left(q^{-A}\right) .
$$

Now, we write $\sum_{\ell} \hat{F}(\ell) w\left(\frac{|\ell|}{L}\right)=T_{1}-T_{2}$, where

$$
T_{1}:=\sum_{\ell} w\left(\frac{|\ell|}{L}\right) \mathcal{K} \int_{0}^{\infty} \frac{1}{u} \mathrm{e}\left(\ell u+\frac{\alpha y}{u}+\frac{\beta z}{u}\right) J_{k-1}\left(\frac{4 \pi \sqrt{\alpha \beta y z}}{u}\right) d u
$$

and

$$
T_{2}:=\sum_{\ell} w\left(\frac{|\ell|}{L}\right) \mathcal{K} \int_{0}^{\infty} \frac{1-\eta(u)}{u} \mathrm{e}\left(\ell u+\frac{\alpha y}{u}+\frac{\beta z}{u}\right) J_{k-1}\left(\frac{4 \pi \sqrt{\alpha \beta y z}}{u}\right) d u
$$


We use (5.7) to evaluate $T_{1}$ and obtain

$$
T_{1}=2 \pi \sum_{\ell=1}^{\infty} w\left(\frac{\ell}{L}\right) J_{k-1}(4 \pi \sqrt{\alpha y \ell}) J_{k-1}(4 \pi \sqrt{\beta z \ell}) .
$$

For $T_{2}$, we note that $\xi(u)=1-\eta(u)=1$ if $0<s<1 / 4,0 \leq \xi(u) \leq 1$ if $1 / 4 \leq s \leq 1 / 2$, and $\xi(u)=0$ if $s>1 / 2$. Interchanging the sum over $\ell$ and the integration over $u$ and applying Poisson summation formula, we have

$$
T_{2}=\mathcal{K} \int_{0}^{\infty} \frac{\xi(u)}{u} \mathrm{e}\left(\frac{\alpha y}{\delta}+\frac{\beta z}{\delta}\right) J_{k-1}\left(\frac{4 \pi \sqrt{\alpha \beta y z}}{u}\right) \sum_{\ell} L \hat{w}(L(\ell+u)) d u .
$$

Since $\hat{w}(y) \ll(1+|y|)^{-A}$, the main contribution comes from $\ell=0$ and $0 \leq u<1 / 4$. Therefore

$$
\begin{aligned}
T_{2} & =\mathcal{K} \int_{0}^{\infty} \frac{\xi(u)}{u} \mathrm{e}\left(\frac{\alpha y}{\delta}+\frac{\beta z}{\delta}\right) J_{k-1}\left(\frac{4 \pi \sqrt{\alpha \beta y z}}{u}\right) L \hat{w}(L u) d u+O\left(q^{-A}\right) \\
& =\mathcal{K} \int_{0}^{\infty} \frac{1}{u} \mathrm{e}\left(\frac{\alpha y}{\delta}+\frac{\beta z}{\delta}\right) J_{k-1}\left(\frac{4 \pi \sqrt{\alpha \beta y z}}{u}\right) L \hat{w}(L u) d u+O\left(q^{-A}\right) \\
& =2 \pi \int_{0}^{\infty} w\left(\frac{\ell}{L}\right) J_{k-1}(4 \pi \sqrt{\alpha y \ell}) J_{k-1}(4 \pi \sqrt{\beta z \ell}) d \ell+O\left(q^{-A}\right),
\end{aligned}
$$

where the last equality comes from Plancherel's formula and (5.7).

Next, the following lemma deals with the sum and the integral involving $\ell$.

Lemma 7.3. Let $w$ be a smooth function on $\mathbb{R}^{+}$with $w(x)=1$ if $0 \leq x \leq 1$, and $w(x)=0$ if $x>2$. Also we let $\gamma$ be a complex number where $\operatorname{Re} \gamma \ll \frac{1}{\log q}$, $\operatorname{Re} \gamma<0$ and $L=q^{100}$. Then

$$
\sum_{\ell=1}^{\infty} w\left(\frac{\ell}{L}\right) \frac{1}{\ell^{1+\gamma}}-\int_{0}^{\infty} w\left(\frac{\ell}{L}\right) \frac{1}{\ell^{1+\gamma}} d \ell=\zeta(1+\gamma)+O\left(q^{-20}\right) .
$$

Proof. Let $\tilde{w}(z)$ be the Mellin transform of $w$, defined by

$$
\tilde{w}(z)=\int_{0}^{\infty} w(t) \frac{t^{z}}{t} d t
$$

From the definition, $\tilde{w}(z)$ is analytic for Re $z>0$, and integration by parts gives

$$
\tilde{w}(z)=-\frac{1}{z} \int_{0}^{\infty} w^{\prime}(t) t^{z} d t
$$

so $\tilde{w}(z)$ can be analytically continued to $\operatorname{Re} z>-1$ except at $z=0$ where it has a simple pole with residue $w(0)=1$. For $\sigma>\min \{0,-\operatorname{Re} \gamma\}$, we have

$$
\begin{aligned}
\sum_{\ell=1}^{\infty} w\left(\frac{\ell}{L}\right) \frac{1}{\ell^{1+\gamma}} & =\sum_{\ell=1}^{\infty} \frac{1}{2 \pi i} \int_{(\sigma)} \tilde{w}(z)\left(\frac{\ell}{L}\right)^{-z} \frac{1}{\ell^{1+\gamma}} d z \\
& =\frac{1}{2 \pi i} \int_{(\sigma)} \tilde{w}(z) L^{z} \zeta(1+\gamma+z) d z
\end{aligned}
$$


Shifting the contour to $\operatorname{Re}(z)=-1 / 4$, we have that (7.4) is

$$
\zeta(1+\gamma)+\tilde{w}(-\gamma) L^{-\gamma}+O\left(q^{-20}\right)
$$

The Lemma follows from noting that $\tilde{w}(-\gamma) L^{-\gamma}=\int_{0}^{\infty} w\left(\frac{\ell}{L}\right) \frac{1}{\ell^{1+\gamma}} d \ell$.

7.2. Calculation of residues. In this section, we will calculate

$$
\operatorname{Res}_{s_{1}=1-\alpha_{1}} \operatorname{Res}_{s_{2}=1+\beta_{1}}\left(\mathcal{T}_{M, \boldsymbol{\alpha}, \boldsymbol{\beta}}^{+}\left(c, x, s_{1}, s_{2}\right)+\mathcal{T}_{M, \boldsymbol{\alpha}, \boldsymbol{\beta}}^{-}\left(c, x, s_{1}, s_{2}\right)\right) .
$$

To do this, we essentially need to consider

$$
\underset{s_{1}=1-\alpha_{1}}{\operatorname{Res}} D_{3}\left(s_{1}, \pm \frac{\lambda_{1}}{\eta_{1}}, \boldsymbol{\alpha}\right) y^{s_{1}-1}
$$

where $\frac{\lambda_{1}}{\eta_{1}}$ is defined in (6.1). Let $\left(a_{1} b_{1}, a_{2} b_{2}\right)=\lambda, a_{1} b_{1}=u_{1} \lambda, a_{2} b_{2}=u_{2} \lambda$, where $\left(u_{1}, u_{2}\right)=1$. Note that $\left(u_{1} x-u_{2}, c\right)=\left(u_{2} \bar{x}-u_{1}, c\right)=\delta$. Hence

$$
\delta_{1}:=\left(\left(a_{2} b_{2} \bar{x}-a_{1} b_{1}\right) a_{1}, a_{2} b_{2} c\right)=\lambda\left(\left(u_{2} \bar{x}-u_{1}\right) a_{1}, u_{2} c\right)=\lambda \delta\left(a_{1}, u_{2} c / \delta\right),
$$

and $\lambda_{1}=\bar{q}\left(a_{2} b_{2} \bar{x}-a_{1} b_{1}\right) a_{1} / \delta_{1}$ and $\eta_{1}=a_{2} b_{2} c / \delta_{1}$.

By (B.4), we obtain that

$$
\begin{aligned}
\mathcal{R}_{1}\left(\frac{c}{\delta}, \mathbf{a}, \mathbf{b}\right) & :=\operatorname{Res}_{s_{1}=1-\alpha_{1}} D_{3}\left(s_{1}, \pm \frac{\lambda_{1}}{\eta_{1}}, \boldsymbol{\alpha}\right) \\
& =\frac{1}{\eta_{1}^{2-2 \alpha_{1}+\alpha_{2}+\alpha_{3}}} \sum_{\substack{1 \leq a_{1}, a_{2} \leq \eta_{1} \\
\eta_{1} \mid a_{1} a_{2}}} \sum \zeta\left(1-\alpha_{1}+\alpha_{2}, \frac{a_{2}}{\eta_{1}}\right) \zeta\left(1-\alpha_{1}+\alpha_{3}, \frac{a_{3}}{\eta_{1}}\right) .
\end{aligned}
$$

Hence

$$
\operatorname{Res}_{s_{1}=1-\alpha_{1}} D_{3}\left(s_{1}, \pm \frac{\lambda_{1}}{\eta_{1}}, \boldsymbol{\alpha}\right) y^{s_{1}-1}=\mathcal{R}_{1}\left(\frac{c}{\delta}, \mathbf{a}, \mathbf{b}\right) y^{-\alpha}
$$

Similarly, we let $\mathcal{R}_{2}(c, \mathbf{a}, \mathbf{b}):=\operatorname{Res}_{s_{2}=1+\beta_{1}} D_{3}\left(s_{2}, \pm \frac{\lambda_{2}}{\eta_{2}},-\boldsymbol{\beta}\right)$. Then

$$
\mathcal{R}_{2}\left(\frac{c}{\delta}, \mathbf{a}, \mathbf{b}\right)=\frac{1}{\eta_{2}^{2+2 \beta_{1}-\beta_{2}-\beta_{3}}} \sum_{\substack{1 \leq a_{1}, a_{2} \leq \eta_{2} \\ \eta_{2} \mid a_{1} a_{2}}} \zeta\left(1+\beta_{1}-\beta_{2}, \frac{a_{2}}{\eta_{2}}\right) \zeta\left(1+\beta_{1}-\beta_{3}, \frac{a_{3}}{\eta_{2}}\right)
$$

and

$$
\operatorname{Res}_{s_{2}=1+\beta_{1}} D_{3}\left(s_{1}, \pm \frac{\lambda_{2}}{\eta_{2}}, \boldsymbol{\alpha}\right) z^{s_{2}-1}=\mathcal{R}_{2}\left(\frac{c}{\delta}, \mathbf{a}, \mathbf{b}\right) z^{\beta}
$$


7.3. Computing $R_{\alpha_{1}, \beta_{1}}$. From the previous section, $R_{\alpha_{1}, \beta_{1}}$ can be written as

$R_{\alpha_{1}, \beta_{1}}=\frac{2 \pi}{q} \sum_{\substack{a_{1}, b_{1}, a_{2}, b_{2} \geq 1 \\\left(a_{1} a_{2} b_{1} b_{2}, q\right)=1}} \sum_{a_{1}} \frac{\mathscr{B}\left(a_{1}, b_{1} ; \boldsymbol{\alpha}\right)}{a_{1}^{\frac{3}{2}} b_{1}} \frac{\mathscr{B}\left(a_{2}, b_{2} ;-\boldsymbol{\beta}\right)}{a_{2}^{\frac{3}{2}} b_{2}} \sum_{M}^{d} \sum_{N}^{d} \mathcal{A}(\mathbf{a}, \mathbf{b}, M, N)+O\left(q^{-1 / 2+\varepsilon}\right)$

where $\mathcal{A}(\mathbf{a}, \mathbf{b}, M, N)$ is defined as

$$
\sum_{c=1}^{\infty} \frac{\mathcal{F}(c)}{c} \sum_{x(\bmod c)}^{*} \mathcal{R}_{1}\left(\frac{c}{\delta}, \mathbf{a}, \mathbf{b}\right) \mathcal{R}_{2}\left(\frac{c}{\delta}, \mathbf{a}, \mathbf{b}\right)
$$

and

$$
\begin{aligned}
\mathcal{F}(c):=\mathcal{F}_{\boldsymbol{\alpha}, \boldsymbol{\beta}}(c, \mathbf{a}, \mathbf{b}):=\int_{0}^{\infty} \int_{0}^{\infty} & \frac{1}{y^{1 / 2+\alpha_{1}}} \frac{1}{z^{1 / 2-\beta_{1}}} V_{\boldsymbol{\alpha}, \boldsymbol{\beta}}\left(a_{1}^{3} b_{1}^{2} y, a_{2}^{3} b_{2}^{2} z ; q\right) J_{k-1}\left(\frac{4 \pi \sqrt{a_{2} y a_{1} z}}{c q}\right) \times \\
& \times f\left(\frac{y}{N}\right) f\left(\frac{z}{M}\right) \mathcal{K} \mathrm{e}\left(\frac{a_{1}^{2} b_{1} y}{c q a_{2} b_{2}}+\frac{a_{2}^{2} b_{2} z}{c q a_{1} b_{1}}\right) d y d z .
\end{aligned}
$$

We remark that we can extend the sum over $c$ to all positive integers in a similar manner as in the truncation argument in Proposition 5.2. Now, we let

$$
\frac{1}{c^{2}} \mathcal{G}(c, \mathbf{a}, \mathbf{b}):=\mathcal{R}_{1}(c, \mathbf{a}, \mathbf{b}) \mathcal{R}_{2}(c, \mathbf{a}, \mathbf{b})
$$

so that we can write the sum over $c$ in $(7.5)$ as

$$
\begin{aligned}
& \sum_{c=1}^{\infty} \frac{\mathcal{F}(c)}{c} \sum_{\delta \mid c} \sum_{\substack{x(\bmod c) \\
\left(u_{1} x-u_{2}, c\right)=\delta}}^{*} \frac{1}{(c / \delta)^{2}} \mathcal{G}\left(\frac{c}{\delta}, \mathbf{a}, \mathbf{b}\right)=\sum_{\delta=1}^{\infty} \frac{1}{\delta} \sum_{c=1}^{\infty} \frac{\mathcal{F}(c \delta)}{c} \sum_{\substack{x(\bmod c \delta) \\
\left(u_{1} x-u_{2}, c \delta\right)=\delta}}^{*} \frac{\mathcal{G}(c, \mathbf{a}, \mathbf{b})}{c^{2}} \\
& =\sum_{\delta=1}^{\infty} \frac{1}{\delta} \sum_{c=1}^{\infty} \frac{\mathcal{F}(c \delta)}{c} \sum_{x(\bmod c \delta)}^{*} \sum_{b \mid\left(\frac{u_{1} x-u_{2}}{\delta}, c\right)} \frac{\mu(b) \mathcal{G}(c, \mathbf{a}, \mathbf{b})}{c^{2}} \\
& =\sum_{\substack{\delta \geq 1 \\
\left(\delta, u_{1} u_{2}\right)=1}} \frac{1}{\delta} \sum_{\substack{b \geq 1 \\
\left(b, u_{1} u_{2}\right)=1}} \frac{\mu(b)}{b^{3}} \sum_{c \geq 1} \frac{\mathcal{G}(c b, \mathbf{a}, \mathbf{b}) \mathcal{F}(c b \delta)}{c^{3}} \sum_{\substack{x(\bmod c \delta b) \\
x \equiv u_{2} \bar{u}_{1}(\bmod b \delta)}}^{*} 1,
\end{aligned}
$$


where the sum over $x$ is 0 if $\left(u_{1} u_{2}, b \delta\right) \neq 1$ since $\left(u_{1}, u_{2}\right)=1$. Applying Lemma 7.1 to the sum over $x$, we then obtain that

$$
\begin{aligned}
& \mathcal{A}(\mathbf{a}, \mathbf{b}, M, N)=\sum_{\substack{\delta \geq 1 \\
\left(\delta, u_{1} u_{2}\right)=1}} \frac{1}{\delta} \sum_{\substack{b \geq 1 \\
\left(b, u_{1} u_{2}\right)=1}} \frac{\mu(b)}{b^{3}} \sum_{c \geq 1} \frac{1}{c^{2}} \prod_{\substack{p \mid c \\
p \nmid b \delta}}\left(1-\frac{1}{p}\right) \mathcal{F}(c b \delta) \mathcal{G}(c b, \mathbf{a}, \mathbf{b}) \\
& =\sum_{\substack{h \geq 1 \\
h \mid u_{1} u_{2}}} \frac{\mu(h)}{h} \sum_{\delta \geq 1} \frac{1}{\delta} \sum_{\substack{b \geq 1 \\
\left(b, u_{1} u_{2}\right)=1}} \frac{\mu(b)}{b^{3}} \sum_{c \geq 1} \frac{1}{c^{2}} \prod_{\substack{p \mid c \\
p \nmid b h \delta}}\left(1-\frac{1}{p}\right) \mathcal{F}(c b h \delta) \mathcal{G}(c b, \mathbf{a}, \mathbf{b}) \\
& =\sum_{\substack{h \geq 1 \\
h \mid u_{1} u_{2}}} \frac{\mu(h)}{h} \sum_{\substack{b \geq 1 \\
\left(b, u_{1} u_{2}\right)=1}} \frac{\mu(b)}{b^{3}} \sum_{c \geq 1} \frac{\mathcal{G}(c b, \mathbf{a}, \mathbf{b})}{c^{2}} \sum_{\gamma \mid c} \prod_{\substack{p \mid c \\
p \nmid b h \gamma}}\left(1-\frac{1}{p}\right) \sum_{\delta \geq 1} \frac{1}{\delta} \mathcal{F}(c b h \delta) \sum_{g \mid(c / \gamma, \delta / \gamma)} \mu(g) \\
& =\sum^{\#} \mathscr{G}_{\mathbf{a}, \mathbf{b}}(1 ; h, b, c, \gamma, g) \sum_{\delta \geq 1} \frac{1}{\delta} \mathcal{F}(\operatorname{cbh} g \gamma \delta),
\end{aligned}
$$

where

$$
\sum^{\#} \mathscr{G}_{\mathbf{a}, \mathbf{b}}(s ; h, b, c, \gamma, g)=\sum_{\substack{h \geq 1 \\ h \mid u_{1} u_{2}}} \frac{\mu(h)}{h^{s}} \sum_{\substack{b \geq 1 \\\left(b, u_{1} u_{2}\right)=1}} \frac{\mu(b)}{b^{2+s}} \sum_{c \geq 1} \frac{\mathcal{G}(c b, \mathbf{a}, \mathbf{b})}{c^{1+s}} \sum_{\gamma \mid c} \frac{1}{\gamma^{s}} \sum_{g \mid \frac{c}{\gamma}} \frac{\mu(g)}{g^{s}} \prod_{\substack{p \mid c \\ p \nmid b h \gamma}}\left(1-\frac{1}{p}\right) .
$$

Next applying Lemma 7.2 to the sum over $\delta$, and summing $\sum_{M}^{d} \sum_{N}^{d}$, we have that

$$
\begin{aligned}
& \sum_{M}^{d} \sum_{N}^{d} \mathcal{A}(\mathbf{a}, \mathbf{b}, M, N) \\
& =2 \pi \frac{1}{2 \pi i}\left(\frac{q}{4 \pi^{2}}\right)^{\delta(\boldsymbol{\alpha}, \boldsymbol{\beta})} \int_{-\infty}^{\infty} \int_{(1)} \sum^{\#} \mathscr{G}_{\mathbf{a}, \mathbf{b}}(1 ; h, b, c, \gamma, g)\left\{\sum_{\ell=1}^{\infty} w\left(\frac{\ell}{L}\right)-\int_{0}^{\infty} w\left(\frac{\ell}{L}\right) d \ell\right\} \\
& \quad \times G\left(\frac{1}{2}+s ; \boldsymbol{\alpha}+i t, \boldsymbol{\beta}+i t\right) H(s ; \boldsymbol{\alpha}, \boldsymbol{\beta})\left(\frac{a_{2}^{3} b_{2}^{2}}{a_{1}^{3} b_{1}^{2}}\right)^{i t}\left(a_{1}^{3} b_{1}^{2} a_{2}^{3} b_{2}^{2}\right)^{-s} \frac{q^{3 s}}{\left(4 \pi^{2}\right)^{3 s}} \\
& \quad \times \int_{0}^{\infty} \int_{0}^{\infty} \frac{y^{-\frac{1}{2}-\alpha_{1}}}{y^{s+i t}} \frac{z^{-\frac{1}{2}+\beta_{1}}}{z^{s-i t}} J_{k-1}\left(4 \pi \sqrt{\frac{a_{1}^{2} b_{1} y \ell}{a_{2} b_{2} c b h g \gamma q}}\right) J_{k-1}\left(4 \pi \sqrt{\frac{a_{2}^{2} b_{2} z \ell}{a_{1} b_{1} c b h g \gamma q}}\right) d y d z \frac{d s}{s} d t .
\end{aligned}
$$

The integration over $y$ and $z$ can be evaluated by Equation 707.14 in [9], which is

$$
\int_{0}^{\infty} v^{\mu}(v k)^{\frac{1}{2}} J_{\nu}(v k) d v=2^{\mu+1 / 2} k^{-\mu-1} \frac{\Gamma\left(\frac{\mu}{2}+\frac{\nu}{2}+\frac{3}{4}\right)}{\Gamma\left(\frac{\nu}{2}-\frac{\mu}{2}+\frac{1}{4}\right)}
$$


for $-\operatorname{Re} \nu-\frac{3}{2}<\operatorname{Re} \mu<0$. Then we apply Lemma 7.3 to the sum and the integration over $\ell$. Therefore after summing over $a_{1}, a_{2}, b_{1}, b_{2}$, we obtain that the main term of $R_{\alpha_{1}, \beta_{1}}$ is

$$
\begin{aligned}
\frac{1}{2 \pi i}\left(\frac{q}{4 \pi^{2}}\right)^{\delta(\pi(\boldsymbol{\alpha}), \pi(\boldsymbol{\beta}))} & \int_{-\infty}^{\infty} \int_{(\varepsilon)} G_{\alpha_{1}, \beta_{1}}\left(\frac{1}{2}+s ; \boldsymbol{\alpha}+i t, \boldsymbol{\beta}+i t\right) H(s ; \boldsymbol{\alpha}, \boldsymbol{\beta}) \mathcal{M}_{\alpha_{1}, \beta_{1}}(s) \\
& \times \zeta\left(1-\alpha_{1}+\beta_{1}-2 s\right) \frac{q^{s}}{\left(4 \pi^{2}\right)^{s}} \frac{d s}{s} d t
\end{aligned}
$$

where $(\pi(\alpha), \pi(\beta))=\left(\beta_{1}, \alpha_{2}, \alpha_{3} ; \alpha_{1}, \beta_{2}, \beta_{3}\right)$,

$G_{\alpha_{i}, \beta_{j}}\left(\frac{1}{2}+s ; \boldsymbol{\alpha}, \boldsymbol{\beta}\right)=\Gamma\left(\frac{k}{2}-s-\alpha_{i}\right) \Gamma\left(\frac{k}{2}-s+\beta_{j}\right) \prod_{\ell \neq i} \Gamma\left(\frac{k}{2}+s+\alpha_{\ell}\right) \prod_{\ell \neq j} \Gamma\left(\frac{k}{2}+s-\beta_{\ell}\right)$,

and

$$
\begin{aligned}
\mathcal{M}_{\alpha_{1}, \beta_{1}}(s):= & \sum \sum_{\substack{a_{1}, b_{1}, a_{2}, b_{2} \geq 1 \\
\left(a_{1} a_{2} b_{1} b_{2}, q\right)=1}} \sum_{1} \frac{\mathscr{B}\left(a_{1}, b_{1} ; \boldsymbol{\alpha}\right)}{a_{1}^{2-2 \alpha_{1}-\beta_{1}+2 s} b_{1}^{1-\alpha_{1}-\beta_{1}+2 s}} \frac{\mathscr{B}\left(a_{2}, b_{2} ;-\boldsymbol{\beta}\right)}{a_{2}^{2+\alpha_{1}+2 \beta_{1}+2 s} b_{2}^{1+\alpha_{1}+\beta_{1}+2 s}} \\
& \times \sum^{\#} \mathscr{G}_{\mathbf{a}, \mathbf{b}}\left(2 s+\alpha_{1}-\beta_{1} ; h, b, c, \gamma, g\right) .
\end{aligned}
$$

Now, in the ensuing discussion, we temporarily assume that $\operatorname{Re}\left(\alpha_{1}\right)<\operatorname{Re}\left(\alpha_{2}\right), \operatorname{Re}\left(\alpha_{3}\right)$ and $\operatorname{Re}\left(\beta_{1}\right)>\operatorname{Re}\left(\beta_{2}\right), \operatorname{Re}\left(\beta_{3}\right)$. In this region,

$$
\mathcal{R}_{1}\left(\frac{c}{\delta}, \mathbf{a}, \mathbf{b}\right)=\sum_{\substack{n_{2}, n_{3} \geq 1 \\ u_{2} \geq 1 \\ \delta\left(a_{1}, u_{2} c / \delta\right)}} \frac{1}{n_{2} n_{3}}
$$

and

$$
\underset{s_{1}=1-\alpha_{i}}{\operatorname{Res}} D_{3}\left(s_{1}, \pm \frac{\lambda_{1}}{\eta_{1}}, \boldsymbol{\alpha}\right) y^{s_{1}-1}=\mathcal{R}_{1}\left(\frac{c}{\delta}, \mathbf{a}, \mathbf{b}\right) y^{-\alpha_{1}}=y^{-\alpha_{1}} \sum_{\substack{n_{2}, n_{3} \geq 1 \\ \frac{u_{2}}{\delta\left(a_{1}, u_{2} c / \delta\right)} \mid n_{2} n_{3}}} \frac{1}{n_{2}^{1+\alpha_{2}-\alpha_{1}} n_{3}^{1+\alpha_{3}-\alpha_{1}}} .
$$

Similarly,

$$
\operatorname{Res}_{s_{2}=1+\beta_{1}} D_{3}\left(s_{2}, \pm \frac{\lambda_{2}}{\eta_{2}},-\boldsymbol{\beta}\right) z^{s_{2}-1}=\mathcal{R}_{2}\left(\frac{c}{\delta}, \mathbf{a}, \mathbf{b}\right) z^{\beta_{1}}=z^{\beta_{1}} \sum_{\substack{m_{2}, m_{3} \geq 1 \\ \text { unc } \\ \delta\left(a_{2}, u_{1} c / \delta\right)}} \sum_{m_{2} m_{3}} \frac{1}{m_{2}^{1+\beta_{1}-\beta_{2}} m_{3}^{1+\beta_{1}-\beta_{3}}} .
$$

Thus,

$$
\begin{aligned}
& \frac{1}{c^{2}} \mathcal{G}(c, \mathbf{a}, \mathbf{b})=\sum_{\substack{n_{2}, n_{3} \geq 1 \\
\frac{u_{2} c}{\left(a_{1}, u_{2} c\right)} \mid n_{2} n_{3}}} \frac{1}{n_{2}^{1+\alpha_{2}-\alpha_{1}} n_{3}^{1+\alpha_{3}-\alpha_{1}}} \sum_{\substack{m_{2}, m_{3} \geq 1 \\
\frac{u_{1} c}{\left(a_{2}, u_{1} c\right)} \mid m_{2} m_{3}}} \frac{1}{m_{2}^{1+\beta_{1}-\beta_{2}} m_{3}^{1+\beta_{1}-\beta_{3}}} \\
& =\frac{\left(a_{1}, u_{2} c\right)\left(a_{2}, u_{1} c\right)}{u_{1} u_{2} c^{2}} \sum_{n=1}^{\infty} \frac{\sigma_{2}\left(\frac{u_{2} c}{\left(a_{1}, u_{2} c\right)} ; \alpha_{2}-\alpha_{1}, \alpha_{3}-\alpha_{1}\right)}{n} \sum_{m=1}^{\infty} \frac{\sigma_{2}\left(\frac{u_{1} c m}{\left(a_{2}, u_{1} c\right)} ; \beta_{1}-\beta_{2}, \beta_{1}-\beta_{3}\right)}{m} .
\end{aligned}
$$


From this, we may then check that

$$
\mathcal{M}_{\alpha_{1}, \beta_{1}}(s)=\prod_{j=2}^{3} \zeta\left(1+2 s+\alpha_{j}-\beta_{1}\right) \zeta\left(1+2 s+\alpha_{1}-\beta_{j}\right) \mathcal{J}_{\alpha_{1}, \beta_{1}}(s),
$$

where $\mathcal{J}_{\alpha_{1}, \beta_{1}}$ is absolutely convergent in the region $\operatorname{Re}(s)=-1 / 4+\varepsilon$. Although we have a priori only verified (7.10) for the region $\operatorname{Re}\left(\alpha_{1}\right)<\operatorname{Re}\left(\alpha_{2}\right), \operatorname{Re}\left(\alpha_{3}\right)$ and $\operatorname{Re}\left(\beta_{1}\right)>$ $\operatorname{Re}\left(\beta_{2}\right), \operatorname{Re}\left(\beta_{3}\right)$, we see that (7.10) must hold for all values of $\alpha_{i}, \beta_{j}$ by analytic continuation.

We note that the pole of $\zeta\left(1-\alpha_{1}+\beta_{1}-2 s\right)$ at $s=\left(\alpha_{1}-\beta_{1}\right) / 2$ and the poles of $\zeta\left(1+2 s+\alpha_{i}-\beta_{j}\right)$ at $s=\left(\alpha_{i}-\beta_{j}\right) / 2$ cancel with the zeros at the same point from $H(s ; \boldsymbol{\alpha}, \boldsymbol{\beta})$. Thus, the integrand in (7.7) has only a simple pole at $s=0$ and is analytic for all values of $s$ with $\operatorname{Re} s>-1 / 4+\epsilon$. Moving the line of integration to $\operatorname{Re}(s)=-1 / 4+\varepsilon$, we then obtain the main term

$\zeta\left(1-\alpha_{1}+\beta_{1}\right) \mathcal{M}_{\alpha_{1}, \beta_{1}}(0)\left\{\left(\frac{q}{4 \pi^{2}}\right)^{\delta(\pi(\boldsymbol{\alpha}), \pi(\boldsymbol{\beta}))} H(0 ; \boldsymbol{\alpha}, \boldsymbol{\beta}) \int_{-\infty}^{\infty} G\left(\frac{1}{2} ; \pi(\boldsymbol{\alpha})+i t, \pi(\boldsymbol{\beta})+i t\right) d t\right\}$,

with negligible error term. To finish the proof of Proposition 6.1, we will show that the local factor at prime $p$ of the Euler product of $\zeta\left(1-\alpha_{1}+\beta_{1}\right) \mathcal{M}_{\alpha_{1}, \beta_{1}}(0)$ is the same as the one in $\mathcal{A} \mathcal{Z}\left(\frac{1}{2} ; \pi(\boldsymbol{\alpha}), \pi(\boldsymbol{\beta})\right)$ defined in (2.16) and (2.17). The details of this are in Appendix A.

\section{Proof of Proposition 6.2}

To prove the proposition, it suffices to show that $\mathcal{E}_{i}^{+}(q ; \boldsymbol{\alpha}, \boldsymbol{\beta}) \ll q^{-1 / 4+\varepsilon}$ for $i=1$ and $i=5$ since the proofs of upper bounds for other terms are similar. We start with a lemma that will be used in the proof.

Lemma 8.1. Let $\lambda, \eta$ be integers such that $(\lambda, \eta)=1$ and $\lambda, \eta \ll q^{A}$, where $A$ is a fixed constant. Moreover, for $i, j=1,2,3, \alpha_{i} \ll \frac{1}{\log q}$, and $\left|\alpha_{i}-\alpha_{j}\right| \gg \frac{1}{q^{\varepsilon_{1}}}$ when $i \neq j$. Then for $\varepsilon>\varepsilon_{1}$,

$$
\operatorname{Res}_{s=1-\alpha_{i}} D_{3}\left(s, \frac{\lambda}{\eta}, \boldsymbol{\alpha}\right) \ll \frac{q^{\varepsilon}}{\eta}
$$

where $D_{3}\left(s, \frac{\lambda}{\eta}, \boldsymbol{\alpha}\right)$ is defined in (B.4).

Proof. By symmetry, it suffices to prove the statement for the residue at $1-\alpha_{1}$. For $\operatorname{Re}(s)>1+\operatorname{Re}\left(\alpha_{1}-\alpha_{j}\right)$, where $j=2,3$, let

$$
\begin{aligned}
D(s) & :=\sum_{\substack{m=1 \\
\eta \mid m}}^{\infty} \frac{\sigma_{2}\left(m ; \alpha_{2}-\alpha_{1}, \alpha_{3}-\alpha_{1}\right)}{m^{s}} \\
& =\frac{1}{\eta^{s}} \sum_{d \mid \eta} \frac{\mu(d) \sigma_{2}\left(\frac{\eta}{d} ; \alpha_{2}-\alpha_{1}, \alpha_{3}-\alpha_{1}\right)}{d^{s+\alpha_{2}+\alpha_{3}-2 \alpha_{1}}} \zeta\left(s+\alpha_{2}-\alpha_{1}\right) \zeta\left(s+\alpha_{3}-\alpha_{1}\right),
\end{aligned}
$$


where we have used Lemma 2.1 to derive the last line. Now, $D(s)$ can be continued analytically to the whole complex plane except for poles at $s=1+\alpha_{1}-\alpha_{j}$ for $j=2,3$. Moreover, $D(1) \ll \frac{q^{\varepsilon}}{\eta}$.

For $i=1,2,3$, and $\operatorname{Re}\left(s+\alpha_{i}\right)>1$, the sum in the Lemma can be rewritten as

$$
\frac{1}{\eta^{3 s+\alpha_{1}+\alpha_{2}+\alpha_{3}}} \sum_{r_{1}, r_{2}, r_{3}} \sum_{(\bmod \eta)} \sum_{\mathrm{e}} \mathrm{e}\left(\frac{\lambda r_{1} r_{2} r_{3}}{\eta}\right) \zeta\left(s+\alpha_{1} ; \frac{r_{1}}{\eta}\right) \zeta\left(s+\alpha_{2} ; \frac{r_{2}}{\eta}\right) \zeta\left(s+\alpha_{3} ; \frac{r_{3}}{\eta}\right) .
$$

This sum can be analytically continued to the whole complex plane except for poles at $s=1-\alpha_{i}$ for $i=1,2,3$. After some arrangement, the contribution of the residue at $s=1-\alpha_{1}$ is

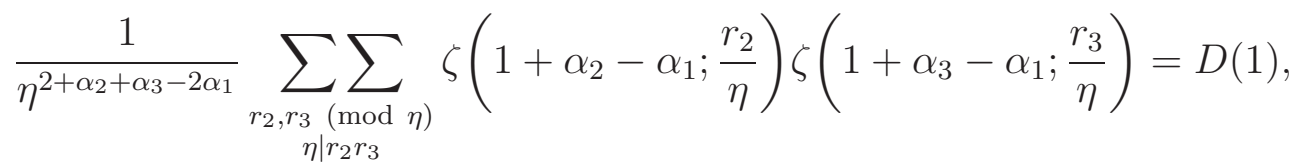

and the Lemma follows.

8.1. Bounding $\mathcal{E}_{1}^{+}(q ; \boldsymbol{\alpha}, \boldsymbol{\beta})$. With the same notation as in Section 7 and $\mathscr{B}$ defined as in $(2.6)$, we recall that

$$
\mathcal{E}_{1, \boldsymbol{\alpha}, \boldsymbol{\beta}}^{+}(q ; \boldsymbol{\alpha}, \boldsymbol{\beta})=\frac{2 \pi}{q} \sum_{\substack{a_{1}, b_{1}, a_{2}, b_{2} \geq 1 \\\left(a_{1} a_{2} b_{1} b_{2}, q\right)=1}} \frac{\mathscr{B}\left(a_{1}, b_{1} ; \boldsymbol{\alpha}\right)}{a_{1}^{\frac{3}{2}} b_{1}} \frac{\mathscr{B}\left(a_{2}, b_{2} ;-\boldsymbol{\beta}\right)}{a_{2}^{\frac{3}{2}} b_{2}} \sum_{M}^{d} \sum_{N}^{d} E_{1, \boldsymbol{\alpha}, \boldsymbol{\beta}}^{+}(\mathbf{a}, \mathbf{b}, M, N),
$$

where

$$
\begin{gathered}
E_{1, \boldsymbol{\alpha}, \boldsymbol{\beta}}^{+}(\mathbf{a}, \mathbf{b}, M, N):=\sum_{c<C} \sum_{x \bmod \delta c}^{*} \frac{\mathcal{T}_{1, \boldsymbol{\alpha}, \boldsymbol{\beta}}^{+}(c, x)}{c}=\sum_{\delta<C} \frac{1}{\delta} \sum_{c<\frac{C}{\delta}} \sum_{\substack{x \bmod \delta c \\
\left(u_{1} x-u_{2}, c \delta\right)=\delta}}^{*} \frac{\mathcal{T}_{1, \boldsymbol{\alpha}, \boldsymbol{\beta}}^{+}(c \delta, x)}{c}, \\
\mathcal{T}_{1, \boldsymbol{\alpha}, \boldsymbol{\beta}}^{+}(c \delta, x):=\frac{\pi^{3 / 2+\alpha_{1}+\alpha_{2}+\alpha_{3}}}{\eta_{1}^{3+\alpha_{1}+\alpha_{2}+\alpha_{3}}} \sum_{i=1}^{3} \operatorname{Res}_{s=1+\beta_{i}} D_{3}\left(s, \frac{\lambda_{2}}{\eta_{2}},-\boldsymbol{\beta}\right) \sum_{n=1}^{\infty} A_{3}\left(n, \frac{\lambda_{1}}{\eta_{1}}, \boldsymbol{\alpha}\right) \mathcal{F}_{1}^{+}(c \delta, n ; \boldsymbol{\alpha}, \boldsymbol{\beta}), \\
\text { for } \lambda_{1}=\frac{\bar{q}\left(u_{2} \bar{x}-u_{1}\right) a_{1}}{\delta\left(a_{1}, u_{2} c\right)}, \eta_{1}=\frac{u_{2} c}{\left(a_{1}, u_{2} c\right)}, \lambda_{2}=\frac{\bar{q}\left(u_{1} x-u_{2}\right) a_{2}}{\delta\left(a_{2}, u_{1} c\right)}, \eta_{2}=\frac{u_{1} c}{\left(a_{2}, u_{1} c\right)}, u_{i}=\frac{a_{i} b_{i}}{\left(a_{1} b_{1}, a_{2} b_{2}\right)} \text { and } \\
\mathcal{F}_{1}^{+}(c \delta, n ; \boldsymbol{\alpha}, \boldsymbol{\beta})=\int_{0}^{\infty} \int_{0}^{\infty} \frac{1}{y^{1 / 2}} \frac{z^{s-1}}{z^{1 / 2}} V_{\boldsymbol{\alpha}, \boldsymbol{\beta}}\left(a_{1}^{3} b_{1}^{2} y, a_{2}^{3} b_{2}^{2} z ; q\right) J_{k-1}\left(\frac{4 \pi \sqrt{a_{2} y a_{1} z}}{c \delta q}\right) f\left(\frac{y}{N}\right) f\left(\frac{z}{M}\right) \times \\
\quad \times i^{-k} \mathrm{e}\left(\frac{a_{1}^{2} b_{1} y}{c \delta q a_{2} b_{2}}+\frac{a_{2}^{2} b_{2} z}{c \delta q a_{1} b_{1}}\right) U_{3}\left(\frac{\pi^{3} n y}{\eta_{1}^{3}} ; \boldsymbol{\alpha}\right) d y d z .
\end{gathered}
$$

We first note that the contribution from the terms $a_{1}^{3} b_{1}^{2} y \gg q^{3 / 2+\varepsilon}$ or $a_{2}^{3} b_{2}^{2} z \gg q^{3 / 2+\varepsilon}$ can be bounded by $q^{-A}$ for any $A$ due to the factor $V_{\alpha, \beta}\left(a_{1}^{3} b_{1}^{2} y, a_{2}^{3} b_{2}^{2} z ; q\right)$. So from now on we assume $a_{1}^{3} b_{1}^{2} N \ll q^{3 / 2+\varepsilon}$ and $a_{2}^{3} b_{2}^{2} M \ll q^{3 / 2+\varepsilon}$.

Moreover, the dyadic sum over $M$ and $N$ contains only $\ll \log ^{2} q$ terms, so it suffices to prove that

$$
E_{1, \boldsymbol{\alpha}, \boldsymbol{\beta}}^{+}(\mathbf{a}, \mathbf{b}, M, N) \ll a_{1}^{1 / 2} q^{3 / 4+\varepsilon},
$$


for fixed $\mathbf{a}$, $\mathbf{b}, M, N$ satisfying $a_{1}^{3} b_{1}^{2} N \ll q^{3 / 2+\varepsilon}$ and $a_{2}^{3} b_{2}^{2} M \ll q^{3 / 2+\varepsilon}$. On a first reading, the reader may set $a_{1}=a_{2}=b_{1}=b_{2}=1$ as this simplifies the notation without substantially changing the calculation.

We now write

$$
E_{1, \boldsymbol{\alpha}, \boldsymbol{\beta}}^{+}(\mathbf{a}, \mathbf{b}, M, N)=H_{1}+H_{2},
$$

where $H_{1}$ is the contribution from the sum over $n \leq \frac{\eta_{1}^{3}}{N} q^{\varepsilon}$, and $H_{2}$ is the rest.

8.1.1. Bounding $H_{1}$. By (B.6), $U_{3}\left(\frac{\pi^{3} n y}{\eta_{1}^{3}}\right) \ll q^{\varepsilon}$ when $n \ll \frac{\eta_{1}^{3} q^{\varepsilon}}{N}$. This and (5.6) gives us that

$$
\mathcal{F}_{1}^{+}(c \delta, n ; \boldsymbol{\alpha}, \boldsymbol{\beta}) \ll M^{1 / 2} N^{1 / 2} q^{\varepsilon} \min \left\{\left(\frac{\sqrt{a_{1} a_{2} M N}}{c \delta q}\right)^{-\frac{1}{2}},\left(\frac{\sqrt{a_{1} a_{2} M N}}{c \delta q}\right)^{k-1}\right\} .
$$

Then, from Lemma 8.1, Lemma B.2, (B.1), and using the fact that $\left(a_{2}, u_{1} c\right) \leq a_{2}$, and $\frac{1}{\left(a_{1}, u_{2} c\right)} \leq 1$, we obtain that $H_{1}$ is bounded by

$$
\begin{aligned}
& M^{\frac{1}{2}} N^{\frac{1}{2}} q^{\varepsilon} \sum_{\delta<C} \sum_{c<\frac{C}{\delta}} \frac{1}{\eta_{1}^{3} \eta_{2}} \sum_{n \ll \frac{\eta_{1}^{3} q^{\varepsilon}}{N}} \sum_{h\left|\eta_{1}, h\right| n^{2}} \eta_{1}^{\frac{3}{2}} \sqrt{h} \min \left\{\left(\frac{\sqrt{a_{1} a_{2} M N}}{c \delta q}\right)^{-\frac{1}{2}},\left(\frac{\sqrt{a_{1} a_{2} M N}}{c \delta q}\right)^{k-1}\right\} \\
& \ll M^{\frac{5}{4}} N^{\frac{1}{4}} q^{\varepsilon} \frac{a_{1}^{\frac{3}{4}} a_{2}^{\frac{7}{4}} u_{2}^{\frac{3}{2}}}{u_{1} q^{\frac{3}{2}}} \ll q^{3 / 4+\varepsilon},
\end{aligned}
$$

as desired. In the above, we have used $\left(a_{1} b_{1}, a_{2} b_{2}\right) \geq 1$, and $a_{1}^{3} b_{1}^{2} N \ll q^{3 / 2+\varepsilon}$ and $a_{2}^{3} b_{2}^{2} M \ll q^{3 / 2+\varepsilon}$.

8.1.2. Bounding $H_{2}$. We start from re-writing $\mathcal{F}_{1}^{+}(c \delta, n ; \boldsymbol{\alpha}, \boldsymbol{\beta})$ as

$$
\int_{-\infty}^{\infty} \int_{\left(\frac{1}{\log q}\right)} V_{1}(s, t) \int_{0}^{\infty} z^{\beta_{1}} F_{s-i t}\left(\frac{z}{M}\right) \mathrm{e}\left(\frac{a_{2}^{2} b_{2} z}{c \delta q a_{1} b_{1}}\right) \mathcal{I}(n, z) d z \frac{d s}{s} d t
$$

where $V_{1}(s, t):=V_{1}(\mathbf{a}, \mathbf{b}, \boldsymbol{\alpha}, \boldsymbol{\beta}, s, t, M, N)$ is defined as

$\frac{1}{2 \pi i}\left(\frac{q}{4 \pi^{2}}\right)^{\delta(\boldsymbol{\alpha}, \boldsymbol{\beta})}\left(\frac{a_{2}^{3} b_{2}^{2}}{a_{1}^{3} b_{1}^{2}}\right)^{i t} G\left(\frac{1}{2}+s ; \boldsymbol{\alpha}+i t, \boldsymbol{\beta}+i t\right) H(s ; \boldsymbol{\alpha}, \boldsymbol{\beta})\left(\frac{q}{4 \pi^{2}}\right)^{3 s} M^{-\frac{1}{2}-s+i t} N^{-\frac{1}{2}-s-i t}$, and $\mathcal{I}(n, z):=\mathcal{I}_{\boldsymbol{\alpha}}(\mathbf{a}, \mathbf{b}, N, n, z, c, \delta)$ is defined as

$$
\int_{0}^{\infty} F_{s+i t}\left(\frac{y}{N}\right) \text { e }\left(\frac{a_{1}^{2} b_{1} y}{c \delta q a_{2} b_{2}}\right) J_{k-1}\left(\frac{4 \pi \sqrt{a_{2} y a_{1} z}}{c \delta q}\right) U_{3}\left(\frac{\pi^{3} n y}{\eta_{1}^{3}} ; \boldsymbol{\alpha}\right) d y
$$

and $F_{v}(x):=\frac{1}{x^{\frac{1}{2}+v}} f(x)$. Note that the $j$-th derivative, $F_{s \pm i t}^{(j)}\left(\frac{y}{N}\right)=O\left(|t|^{j} N^{-j}\right)$.

Note that the trivial bound for $\mathcal{F}_{1}^{+}(c \delta, n ; \boldsymbol{\alpha}, \boldsymbol{\beta})$ is

$$
\mathcal{F}_{1}^{+}(c \delta, n ; \boldsymbol{\alpha}, \boldsymbol{\beta}) \ll \sqrt{M N}(q n)^{\varepsilon} .
$$


There are two cases to consider: (1) $c>\frac{8 \pi \sqrt{a_{1} a_{2} M N}}{\delta q}$, and $(2) c \leq \frac{8 \pi \sqrt{a_{1} a_{2} M N}}{\delta q}$.

Case 1: $c>\frac{8 \pi \sqrt{a_{1} a_{2} M N}}{\delta q}$.

By (5.5), (B.7), and since $\frac{\pi^{3} n y}{\eta_{1}^{3}} \gg q^{\varepsilon}, \mathcal{I}(n, z)$ can be written as

$$
\begin{aligned}
\sum_{j=1}^{K}\left(\frac{\pi^{3} n y}{\eta_{1}^{3}}\right)^{\frac{\beta_{1}+\beta_{2}+\beta_{3}}{3}}\left(\frac{\eta_{1}}{\pi n^{\frac{1}{3}} N^{\frac{1}{3}}}\right)^{j} \sum_{\ell=0}^{\infty} \frac{(-1)^{\ell}}{\ell !(\ell+k-1) !} \int_{0}^{\infty} \mathscr{F}_{j}(y, z, \ell) \mathrm{e}\left(\frac{a_{1}^{2} b_{1} y}{c \delta q a_{2} b_{2}}\right) \times \\
\times\left\{c_{j} \mathrm{e}\left(\frac{3 n^{\frac{1}{3}} y^{\frac{1}{3}}}{\eta_{1}}\right)+d_{j} \mathrm{e}\left(-\frac{3 n^{\frac{1}{3}} y^{\frac{1}{3}}}{\eta_{1}}\right)\right\} d y+O\left(q^{-\varepsilon(K+1)}\right),
\end{aligned}
$$

where $c_{j}, d_{j}$ are some constants, and

$$
\mathscr{F}_{j}(y, z, \ell)=F_{s+i t}\left(\frac{y}{N}\right)\left(\frac{2 \pi \sqrt{a_{2} y a_{1} z}}{c \delta q}\right)^{2 \ell+k-1}\left(\frac{y}{N}\right)^{\frac{-j}{3}}
$$

is supported on $y \in[N, 2 N]$. Moreover, $\frac{\partial^{i} \mathscr{F}_{j}(y, z, \ell)}{\partial y^{i}} \ll \frac{1}{2^{2 \ell}} \frac{|t|^{i}}{N^{i}}$ and $\mathscr{F}_{j}(y, z, \ell) \ll 1$. Thus, picking $K$ large enough so that $q^{-\varepsilon(K+1)}$ is negligible, it suffices to bound integrals of the form

$$
\int_{0}^{\infty} \mathscr{F}_{j}(y, z, \ell) \mathrm{e}\left(\theta_{z}(y, n)\right) d y
$$

where

$$
\theta_{z}(y, n)= \pm \frac{3 n^{\frac{1}{3}} y^{\frac{1}{3}}}{\eta_{1}}+B y, \quad \text { and } \quad B=\frac{a_{1}^{2} b_{1}}{c \delta q a_{2} b_{2}} .
$$

Taking the derivative of $\theta_{z}(y, n)$ with respect to $y$, we have that

$$
\theta_{z}^{\prime}(y, n)=B \pm \frac{n^{\frac{1}{3}}}{y^{\frac{2}{3}} \eta_{1}}
$$

When $n \geq 64\left(B \eta_{1}\right)^{3} N^{2}$ or $n \leq \frac{1}{4}\left(B \eta_{1}\right)^{3} N^{2},\left|\theta_{z}^{\prime}(y, n)\right| \gg \frac{n^{\frac{1}{3}}}{y^{\frac{2}{3}} \eta_{1}} \gg \frac{q^{\varepsilon}}{N}$, since $n \gg \frac{\eta_{1}^{3} q^{\varepsilon}}{N}$. Thus integrating by parts many times shows that the contribution from these terms is negligible. Therefore we only consider the contribution from when $\frac{1}{4}\left(B \eta_{1}\right)^{3} N^{2} \leq n \leq$ $64\left(B \eta_{1}\right)^{3} N^{2}$. Note however that

$$
\left(B \eta_{1}\right)^{3} N^{2} \ll \frac{\left(a_{1}^{2} b_{1}\right)^{3} N^{2}}{\delta^{3} q^{3}} \ll \frac{q^{\varepsilon}}{\delta^{3}},
$$

and that there are no terms of this form unless $N \gg \frac{q^{3 / 2}}{\left(a_{1}^{2} b_{1}\right)^{3 / 2}}$ and $\delta \ll q^{\varepsilon}$. From (8.5), trivially $\mathcal{F}_{1}^{+}(c \delta, n ; \boldsymbol{\alpha}, \boldsymbol{\beta})=O\left(M^{\frac{1}{2}} N^{\frac{1}{2}}(n q)^{\varepsilon}\right)$. Hence the contribution to $H_{2}$ from these 
terms is bounded by

$$
\begin{aligned}
& M^{\frac{1}{2}} N^{\frac{1}{2}} q^{\varepsilon} \sum_{\delta<q^{\varepsilon}} \sum_{c \gg \frac{\sqrt{a_{1} a_{2} M N}}{\delta q}} \frac{1}{\eta_{1}^{\frac{3}{2}} \eta_{2}} \sum_{h \mid \eta_{1}} \sqrt{h} \sum_{\substack{n \ll q^{\varepsilon} \\
h^{2} \mid n}} \frac{\eta_{1}}{n^{\frac{1}{3}} N^{\frac{1}{3}}} \\
& \ll a_{1}^{\frac{5}{4}} b_{1}^{\frac{1}{2}} a_{2}^{\frac{3}{4}} M^{\frac{1}{4}} N^{\frac{1}{4}} q^{\varepsilon} \ll a_{1}^{\frac{1}{2}} q^{\frac{3}{4}+\varepsilon},
\end{aligned}
$$

similar to before.

Case 2: $c \leq \frac{8 \pi \sqrt{a_{1} a_{2} M N}}{\delta q}$.

By (5.4), we write $\mathcal{I}(n, z)$ as

$$
\int_{0}^{\infty}\left\{R^{+}(y, z)+R^{-}(y, z)\right\} \frac{\sqrt{c \delta q}}{\pi\left(a_{1} a_{2} y z\right)^{\frac{1}{4}}} U_{3}\left(\frac{\pi^{3} n y}{\eta_{1}^{3}} ; \boldsymbol{\alpha}\right) \text { e }\left(\frac{a_{1}^{2} b_{1} y}{c \delta q a_{2} b_{2}}\right) d y
$$

where

$$
R^{ \pm}(y, z):=F_{s+i t}\left(\frac{y}{N}\right) W^{ \pm}\left(\frac{4 \pi \sqrt{a_{1} a_{2} y z}}{c \delta q}\right) \mathrm{e}\left( \pm\left(\frac{2 \sqrt{a_{1} a_{2} y z}}{c \delta q}-\frac{k}{4}+\frac{1}{8}\right)\right),
$$

and $W^{+}=W, W^{-}=\bar{W}$.

Similar to Case 1, we explicitly write $U_{3}\left(\frac{\pi^{3} n y}{\eta_{1}^{3}} ; \boldsymbol{\alpha}\right)$ as in Equation (B.7) so it suffices to bound

$$
\begin{aligned}
\sum_{j=1}^{K}\left(\frac{\pi^{3} n y}{\eta_{1}^{3}}\right)^{\frac{\beta_{1}+\beta_{2}+\beta_{3}}{3}} & \left(\frac{\eta_{1}}{\pi n^{\frac{1}{3}} N^{\frac{1}{3}}}\right)^{j} \frac{1}{N^{\frac{1}{4}}} \frac{\sqrt{c \delta q}}{\pi\left(a_{1} a_{2} z\right)^{\frac{1}{4}}} \int_{0}^{\infty} \mathscr{H}_{j}^{ \pm}(y, z)\left\{c_{j} \mathrm{e}\left(\frac{3 n^{\frac{1}{3}} y^{\frac{1}{3}}}{\eta_{1}}\right)+d_{j} \mathrm{e}\left(-\frac{3 n^{\frac{1}{3}} y^{\frac{1}{3}}}{\eta_{1}}\right)\right\} \times \\
& \times \mathrm{e}\left(\frac{a_{1}^{2} b_{1} y}{c \delta q a_{2} b_{2}}\right) \mathrm{e}\left( \pm\left(\frac{2 \sqrt{a_{1} a_{2} y z}}{c \delta q}-\frac{k}{4}+\frac{1}{8}\right)\right) d y+O\left(q^{-\varepsilon(K+1)}\right)
\end{aligned}
$$

where $\mathscr{H}_{j}^{ \pm}(y, z)=\frac{F_{s+i t}\left(\frac{y}{N}\right) W^{ \pm}\left(\frac{4 \pi \sqrt{a_{1} a_{2} y z}}{c \delta q}\right)}{\left(\frac{y}{N}\right)^{\frac{j}{3}+\frac{1}{4}}}$ is supported on $y \in[N, 2 N]$. Note that $\frac{\partial^{(i)} \mathscr{H}_{j}^{ \pm}(y, z)}{\partial y^{i}} \ll_{j, i} \frac{|t|^{i}}{N^{i}}$ and $\mathscr{H}_{j}^{ \pm}(y, z) \ll 1$. Thus, the integration over $y$ is of the form

$$
\int_{0}^{\infty} \mathscr{H}_{j}^{ \pm}(y, z) \mathrm{e}\left(g_{z}(y, n)\right) d y
$$

where

$$
g_{z}(y, n)= \pm \frac{3 n^{\frac{1}{3}} y^{\frac{1}{3}}}{\eta_{1}} \pm\left(2 A \sqrt{y}+\frac{k}{4}-\frac{1}{8}\right)+B y, \quad A=\frac{\sqrt{a_{1} a_{2} z}}{c \delta q}, \quad B=\frac{a_{1}^{2} b_{1}}{c \delta q a_{2} b_{2}} .
$$

Differentiating $g_{z}(y, n)$ with respect to $y$, we have

$$
g_{z}^{\prime}(y, n)= \pm \frac{n^{\frac{1}{3}}}{y^{\frac{2}{3}} \eta_{1}} \pm \frac{A}{y^{\frac{1}{2}}}+B
$$


When $a_{2}^{\frac{3}{2}} b_{2} M^{\frac{1}{2}} \geq 4 a_{1}^{\frac{3}{2}} b_{1} N^{\frac{1}{2}}$, it follows that $\frac{A}{y^{\frac{1}{2}}} \geq \frac{A}{y^{\frac{1}{2}}}-B \geq \frac{1}{2} \frac{A}{y^{\frac{1}{2}}}$ and $\frac{3}{2} \frac{A}{y^{\frac{1}{2}}} \geq \frac{A}{y^{\frac{1}{2}}}+B \geq$ $\frac{A}{y^{\frac{1}{2}}}$. Therefore

$$
\frac{1}{2} \frac{A}{y^{\frac{1}{2}}} \leq\left| \pm \frac{A}{y^{\frac{1}{2}}}+B\right| \leq \frac{3}{2} \frac{A}{y^{\frac{1}{2}}}
$$

When $n \geq 54\left(A \eta_{1}\right)^{3} N^{\frac{1}{2}}$ or $n \leq \frac{1}{64}\left(A \eta_{1}\right)^{3} N^{\frac{1}{2}}$, we have that $\left|g_{z}^{\prime}(y, n)\right| \gg \frac{n^{\frac{1}{3}}}{y^{\frac{2}{3}} \eta_{1}} \gg \frac{q^{\varepsilon}}{N}$, since $n \gg \frac{\eta_{1}^{3} q^{\varepsilon}}{N}$. Integrating by parts many times shows that these terms are negligible. We then consider only the terms when $\frac{1}{64}\left(A \eta_{1}\right)^{3} N^{\frac{1}{2}} \leq n \leq 54\left(A \eta_{1}\right)^{3} N^{\frac{1}{2}}$. Note however that

$$
\left(A \eta_{1}\right)^{3} N^{1 / 2} \ll\left(\frac{\sqrt{a_{1} a_{2} M} u_{2} c}{c \delta q}\right)^{3} N^{1 / 2} \ll\left(\frac{\sqrt{a_{1}}}{\delta q^{\frac{1}{4}}}\right)^{3} N^{1 / 2} \ll \frac{q^{\varepsilon}}{\delta^{3}},
$$

and that the left side is only $\gg 1$ if $N \gg q^{3 / 2} / a_{1}^{3}$ and $\delta \ll q^{\varepsilon}$.

By (8.5), the contribution of $\mathcal{F}_{1}^{+}(c \delta, n ; \boldsymbol{\alpha}, \boldsymbol{\beta})$ to the terms in this range is $O\left(M^{\frac{1}{2}} N^{\frac{1}{2}}(n q)^{\varepsilon}\right)$. So the contribution to $\mathrm{H}_{2}$ from these terms is bounded by

$$
\begin{aligned}
& M^{\frac{1}{2}} N^{\frac{1}{2}} q^{\varepsilon} \sum_{\delta<q^{\varepsilon}} \sum_{c \ll \frac{\sqrt{a_{1} a_{2} M N}}{\delta q}} \frac{1}{\eta_{1}^{\frac{3}{2}} \eta_{2}} \sum_{h \mid \eta_{1}} \sqrt{h} \sum_{\substack{n \ll q^{\varepsilon} \\
h^{2} \mid n}}\left(\frac{\eta_{1}}{n^{\frac{1}{3}} N^{\frac{1}{3}}}\right) \frac{\sqrt{c \delta q}}{\left(a_{1} a_{2} M N\right)^{\frac{1}{4}}} \\
& \ll a_{1}^{\frac{5}{4}} b_{1}^{\frac{1}{2}} a_{2}^{\frac{3}{4}} M^{\frac{1}{4}} N^{\frac{1}{4}} q^{\varepsilon} \ll a_{1}^{\frac{1}{2}} q^{\frac{3}{4}+\varepsilon}
\end{aligned}
$$

which suffices.

When $4 a_{2}^{\frac{3}{2}} b_{2} M^{\frac{1}{2}} \leq a_{1}^{\frac{3}{2}} b_{1} N^{\frac{1}{2}}$, we have that $\frac{1}{2} B<B-\frac{A}{y^{\frac{1}{2}}}<B$ and $\frac{3}{2} B>A y^{\frac{1}{2}}+B>B$. By the same arguments as in Case 1, the range of $n$ that should be considered is of the size $\left(B \eta_{1}\right)^{3} N^{2}$ and give a contribution to $H_{2}$ bounded by $a_{1}^{1 / 2} q^{3 / 4+\varepsilon}$.

When $\frac{1}{4} a_{1}^{\frac{3}{2}} b_{1} N^{\frac{1}{2}}<a_{2}^{\frac{3}{2}} b_{2} M^{\frac{1}{2}}<4 a_{1}^{\frac{3}{2}} b_{1} N^{\frac{1}{2}}$, we have that $\frac{A}{y^{\frac{1}{2}}} \asymp B$, and so the range of $n$ that should be considered is of the size $\left(A \eta_{1}\right)^{3} N^{\frac{1}{2}}$ by the same arguments as above. Hence the contribution from these terms to $H_{2}$ is $O\left(a_{1}^{1 / 4} q^{\frac{3}{4}+\varepsilon}\right)$.

This completes the proof of Proposition 6.2 for $\mathcal{E}_{1}^{+}(q ; \boldsymbol{\alpha}, \boldsymbol{\beta})$. The same proof applies to bound $\mathcal{E}_{i}^{ \pm}(q ; \boldsymbol{\alpha}, \boldsymbol{\beta})$ for $i=2,3,4$.

8.2. Bounding $\mathcal{E}_{5}^{+}(q ; \boldsymbol{\alpha}, \boldsymbol{\beta})$. We first recall that

$$
\mathcal{E}_{5}^{+}(q ; \boldsymbol{\alpha}, \boldsymbol{\beta})=\frac{2 \pi}{q} \sum_{\substack{a_{1}, b_{1}, a_{2}, b_{2} \geq 1 \\\left(a_{1} a_{2} b_{1} b_{2}, q\right)=1}} \sum_{a_{1} b_{1}} \frac{\mathscr{B}\left(a_{1}, b_{1} ; \boldsymbol{\alpha}\right)}{a_{2}^{\frac{3}{2}} b_{2}\left(a_{2}, b_{2} ;-\boldsymbol{\beta}\right)} \sum_{M}^{d} \sum_{N}^{d} E_{5, \boldsymbol{\alpha}, \boldsymbol{\beta}}^{+}(\mathbf{a}, \mathbf{b}, M, N),
$$

where

$$
E_{5, \boldsymbol{\alpha}, \boldsymbol{\beta}}^{+}(\mathbf{a}, \mathbf{b}, M, N):=\sum_{c<C} \sum_{x \bmod \delta c}^{*} \frac{\mathcal{T}_{5, \boldsymbol{\alpha}, \boldsymbol{\beta}}^{+}(c, x)}{c}=\sum_{\delta<C} \frac{1}{\delta} \sum_{c<\frac{C}{\delta}} \sum_{\substack{x \bmod \delta c \\\left(u_{1} x-u_{2}, c \delta\right)=\delta}}^{*} \frac{\mathcal{T}_{5, \boldsymbol{\alpha}, \boldsymbol{\beta}}^{+}(c \delta, x)}{c}
$$




$$
\begin{aligned}
& \mathcal{T}_{5, \boldsymbol{\alpha}, \boldsymbol{\beta}}^{+}(c \delta, x):=\frac{\pi^{3+\sum_{i=1}^{3}\left(\alpha_{i}-\beta_{i}\right)}}{\eta_{1}^{3+\sum_{i=1}^{3} \alpha_{i}} \eta_{2}^{3-\sum_{i=1}^{3} \beta_{i}}} \sum_{n, m \geq 1} \sum_{3} A_{3}\left(n, \frac{\lambda_{1}}{\eta_{1}}, \boldsymbol{\alpha}\right) A_{3}\left(m, \frac{\lambda_{2}}{\eta_{2}},-\boldsymbol{\beta}\right) \mathcal{F}_{5}^{+}(c \delta, n, m ; \boldsymbol{\alpha}, \boldsymbol{\beta}) ; \\
& \text { for } \lambda_{1}=\frac{\bar{q}\left(u_{2} \bar{x}-u_{1}\right) a_{1}}{\delta\left(a_{1}, u_{2} c\right)}, \eta_{1}=\frac{u_{2} c}{\left(a_{1}, u_{2} c\right)}, \lambda_{2}=\frac{\bar{q}\left(u_{1} x-u_{2}\right) a_{2}}{\delta\left(a_{2}, u_{1} c\right)}, \eta_{2}=\frac{u_{1} c}{\left(a_{2}, u_{1} c\right)}, u_{i}=\frac{a_{i} b_{i}}{\left(a_{1} b_{1}, a_{2} b_{2}\right)}, \text { and } \\
& \mathcal{F}_{5}^{+}(c \delta, n, m, \boldsymbol{\alpha}, \boldsymbol{\beta})=\int_{0}^{\infty} \int_{0}^{\infty} \frac{1}{y^{1 / 2}} \frac{1}{z^{1 / 2}} V_{\boldsymbol{\alpha}, \boldsymbol{\beta}}\left(a_{1}^{3} b_{1}^{2} y, a_{2}^{3} b_{2}^{2} z ; q\right) J_{k-1}\left(\frac{4 \pi \sqrt{a_{2} y a_{1} z}}{c \delta q}\right) f\left(\frac{y}{N}\right) f\left(\frac{z}{M}\right) \times \\
& \qquad i^{-k} \mathrm{e}\left(\frac{a_{1}^{2} b_{1} y}{c \delta q a_{2} b_{2}}+\frac{a_{2}^{2} b_{2} z}{c \delta q a_{1} b_{1}}\right) U_{3}\left(\frac{\pi^{3} n y}{\eta_{1}^{3}} ; \boldsymbol{\alpha}\right) U_{3}\left(\frac{\pi^{3} m z}{\eta_{2}^{3}} ;-\boldsymbol{\beta}\right) d y d z .
\end{aligned}
$$

The proofs in this section are very similar to the ones in the previous section. Previously, we had one sum over $n$ and now we have a double sum over $m$ and $n$ which can be treated in a similar manner. To be precise, we begin by dividing $E_{5, \boldsymbol{\alpha}, \boldsymbol{\beta}}^{+}(\mathbf{a}, \mathbf{b}, M, N)$ into $\sum_{i=1}^{4} E_{5, i}^{+}(\mathbf{a}, \mathbf{b}, M, N)$, where $E_{5, i}^{+}(\mathbf{a}, \mathbf{b}, M, N):=E_{5, i, \boldsymbol{\alpha}, \boldsymbol{\beta}}^{+}(\mathbf{a}, \mathbf{b}, M, N)$ is the contribution from case $i$ below.

(1) $n \ll \frac{\eta_{1}^{3} q^{\varepsilon}}{N}$ and $m \ll \frac{\eta_{2}^{3} q^{\varepsilon}}{M}$;

(2) $n \gg \frac{\eta_{1}^{3} q^{\varepsilon}}{N}$ and $m \ll \frac{\eta_{2}^{3} q^{\varepsilon}}{M}$;

(3) $n \ll \frac{\eta_{1}^{3} q^{\varepsilon}}{N}$ and $m \gg \frac{\eta_{2}^{3} q^{\varepsilon}}{M}$;

(4) $n \gg \frac{\eta_{1}^{3} q^{\varepsilon}}{N}$ and $m \gg \frac{\eta_{2}^{3} q^{\varepsilon}}{M}$.

By symmetry, the treatment for cases (2) and (3) is the same, so we will show only the second case.

Similar to Section 8.1, the contribution from the terms $a_{1}^{3} b_{1}^{2} y \gg q^{3 / 2+\varepsilon}$ or $a_{2}^{3} b_{2}^{2} z \gg$ $q^{3 / 2+\varepsilon}$ can be bounded by $q^{-A}$ due to the factor $V_{\boldsymbol{\alpha}, \boldsymbol{\beta}}\left(a_{1}^{3} b_{1}^{2} y, a_{2}^{3} b_{2}^{2} z ; q\right)$. Thus it suffices to prove that

$$
E_{5, i}^{+}(\mathbf{a}, \mathbf{b}, M, N) \ll a_{1}^{1 / 2} a_{2}^{1 / 2} q^{3 / 4+\varepsilon}
$$

for fixed a, b, $M, N$ satisfying $a_{1}^{3} b_{1}^{2} N \ll q^{3 / 2+\varepsilon}$ and $a_{2}^{3} b_{2}^{2} M \ll q^{3 / 2+\varepsilon}$. In fact, we will prove the stronger bound $E_{5, i}^{+}(\mathbf{a}, \mathbf{b}, M, N) \ll a_{1}^{1 / 2} a_{2}^{1 / 2} q^{1 / 2+\varepsilon}$.

8.2.1. Bounding $E_{5,1}^{+}(\mathbf{a}, \mathbf{b}, M, N)$. For this case, $U_{3}\left(\frac{\pi^{3} n y}{\eta_{1}^{3}} ; \boldsymbol{\alpha}\right) \ll q^{\varepsilon}$, and $U_{3}\left(\frac{\pi^{3} m z}{\eta_{2}^{3}} ;-\boldsymbol{\beta}\right) \ll$ $q^{\varepsilon}$ by (B.6). Similar to the arguments in Section 8.1.1, from Lemma 8.1, Lemma B.2 and (5.6), we have that for $k \geq 5, E_{5,1}^{+}(\mathbf{a}, \mathbf{b}, M, N)$ is bounded by 


$$
\begin{aligned}
& \ll M^{\frac{1}{2}} N^{\frac{1}{2}} q^{\varepsilon} \sum_{\delta<C} \sum_{c<\frac{C}{\delta}} \frac{1}{\eta_{1}^{3} \eta_{2}^{3}} \sum_{n \ll \frac{\eta_{1}^{3} q^{\varepsilon}}{N}} \sum_{h_{1}\left|\eta_{1}, h_{1}\right| n^{2}} \eta_{1}^{\frac{3}{2}} \sqrt{h_{1}} \sum_{m \ll \frac{\eta_{2}^{3} q^{\varepsilon}}{M}} \sum_{h_{2}\left|\eta_{2}, h_{2}\right| m^{2}} \eta_{2}^{\frac{3}{2}} \sqrt{h}_{2} \\
& \quad \times \min \left\{\left(\frac{\sqrt{a_{1} a_{2} M N}}{c \delta q}\right)^{-\frac{1}{2}},\left(\frac{\sqrt{a_{1} a_{2} M N}}{c \delta q}\right)^{k-1}\right\} \\
& \ll M^{-\frac{1}{2}} N^{-\frac{1}{2}} q^{\varepsilon} \sum_{\delta<C}\left\{\sum_{\frac{\sqrt{a_{1} a_{2} M N}}{q \delta} \ll c<\frac{C}{\delta}} \eta_{1}^{\frac{3}{2}} \eta_{2}^{\frac{3}{2}}\left(\frac{\sqrt{a_{1} a_{2} M N}}{c \delta q}\right)^{k-1}+\sum_{c \ll \frac{\sqrt{a_{1} a_{2} M N}}{q^{\delta}}} \eta_{1}^{\frac{3}{2}} \eta_{2}^{\frac{3}{2}}\left(\frac{\sqrt{a_{1} a_{2} M N}}{c \delta q}\right)^{-\frac{1}{2}}\right\} \\
& \ll M^{\frac{3}{2}} N^{\frac{3}{2}} q^{\varepsilon} \frac{a_{1}^{2} a_{2}^{2} u_{1}^{\frac{3}{2}} u_{2}^{\frac{3}{2}} \ll q^{\frac{1}{2}+\varepsilon} .}{q^{4}}
\end{aligned}
$$

8.2.2. Bounding $E_{5,2}^{+}(\mathbf{a}, \mathbf{b}, M, N)$. We can write $\mathcal{F}_{5}^{+}(c \delta, n, m ; \boldsymbol{\alpha}, \boldsymbol{\beta})$ as

$$
\int_{-\infty}^{\infty} \int_{\left(\frac{1}{\log q}\right)} V_{1}(s, t) \int_{0}^{\infty} F_{s-i t}\left(\frac{z}{M}\right) \mathrm{e}\left(\frac{a_{2}^{2} b_{2} z}{c \delta q a_{1} b_{1}}\right) U_{3}\left(\frac{\pi^{3} m z}{\eta_{2}^{3}} ;-\boldsymbol{\beta}\right) \mathcal{I}(n, z) d z \frac{d s}{s} d t
$$

where $V_{1}(s, t)$ and $\mathcal{I}(n, z)$ are defined as in (8.3) and (8.4), respectively, and $F_{v}(x)=$ $\frac{1}{x^{\frac{1}{2}+v}} f(x)$. Note that $F_{s \pm i t}^{(j)}\left(\frac{y}{N}\right) \ll|t|^{j} N^{-j}$.

The integration over $z$ can be bounded trivially, and the sum over $m, h_{2}$ can be treated in the same way as in Section 8.2.1. For the integration over $y$, we argue as in Case 1 and 2 of Section 8.1.2 and obtain that $E_{5,2}^{+}(\mathbf{a}, \mathbf{b}, M, N) \ll a_{1}^{\frac{1}{2}} q^{\frac{1}{2}+\varepsilon}$.

\subsubsection{Bounding $E_{5,4}^{+}(\mathbf{a}, \mathbf{b}, M, N)$. We split into two cases as follows.}

Case 1: $c>\frac{8 \pi \sqrt{a_{1} a_{2} M N}}{\delta q}$. We use (5.5) and (B.7), and the integral that we consider is of the form

$$
\int_{0}^{\infty} \int_{0}^{\infty} G(y, z) \mathrm{e}\left(\frac{a_{1}^{2} b_{1} y}{c \delta q a_{2} b_{2}}+\frac{a_{2}^{2} b_{2} z}{c \delta q a_{1} b_{1}} \pm \frac{3 n^{\frac{1}{3}} y^{\frac{1}{3}}}{\eta_{1}} \pm \frac{3 m^{\frac{1}{3}} z^{\frac{1}{3}}}{\eta_{2}}\right) d y d z
$$

where $\frac{\partial^{j} \partial^{i} G(y, z)}{\partial y^{j} \partial z^{i}} \ll \frac{1}{N^{j} M^{i}}, G(x, y) \ll 1$, and it is supported in $[N, 2 N] \times[M, 2 M]$. Therefore, the integration over $y, z$ above is $O(M N)$.

By the same arguments as case 1 of Section 8.1.2, it is sufficient to consider when $c_{1}\left(B_{1} \eta_{1}\right)^{3} N^{2} \ll n \ll c_{2}\left(B_{1} \eta_{1}\right)^{3} N^{2}$ and $c_{1}\left(B_{2} \eta_{2}\right)^{3} M^{2} \ll m \ll c_{2}\left(B_{2} \eta_{2}\right)^{3} M^{2}$, where $c_{1}, c_{2}$ are some constants, $B_{1}=\frac{a_{1}^{2} b_{1}}{c \delta q a_{2} b_{2}}$, and $B_{2}=\frac{a_{2}^{2} b_{2}}{c \delta q a_{1} b_{1}}$, since the terms outside these ranges give negligible contribution from integration by parts many times. By the same arguments as in Section 8.1,

$$
\left(B \eta_{1}\right)^{3} N^{2} \ll \frac{\left(a_{1}^{2} b_{1}\right)^{3} N^{2}}{\delta^{3} q^{3}} \ll \frac{q^{\varepsilon}}{\delta^{3}}, \quad\left(B \eta_{2}\right)^{3} M^{2} \ll \frac{\left(a_{2}^{2} b_{2}\right)^{3} M^{2}}{\delta^{3} q^{3}} \ll \frac{q^{\varepsilon}}{\delta^{3}} .
$$


So there are no terms of this form unless $N \gg \frac{q^{3 / 2}}{\left(a_{1}^{2} b_{1}\right)^{3 / 2}}, M \gg \frac{q^{3 / 2}}{\left(a_{2}^{2} b_{2}\right)^{3 / 2}}$, and $\delta \ll q^{\varepsilon}$. We then obtain that the contribution from these terms to $E_{5,4}^{+}(\mathbf{a}, \mathbf{b}, M, N)$ is bounded by

$$
M^{\frac{1}{2}} N^{\frac{1}{2}} q^{\varepsilon} \sum_{\delta<q^{\varepsilon}} \sum_{c \gg \frac{\sqrt{a_{1} a_{2} M N}}{\delta q}} \frac{1}{\eta_{1}^{\frac{3}{2}}} \eta_{2}^{\frac{3}{2}} \sum_{h_{1} \mid \eta_{1}} \sqrt{h_{1}} \sum_{\substack{n \ll q^{\varepsilon} \\ h_{1}^{2} \mid n}} \sum_{h_{2} \mid \eta_{1}} \sqrt{h_{2}} \sum_{\substack{m \ll q^{\varepsilon} \\ h_{2}^{2} \mid m}} 1 \ll a_{1}^{\frac{1}{2}} a_{2}^{\frac{1}{2}} q^{\frac{1}{2}+\varepsilon} .
$$

Case 2: $c \leq \frac{8 \pi \sqrt{a_{1} a_{2} M N}}{\delta q}$. For this case, we use (5.4), and the integral that we consider is of the form

$$
\frac{\eta_{1} \eta_{2}}{m^{\frac{1}{3}} n^{\frac{1}{3}} M^{\frac{1}{3}} N^{\frac{1}{3}}} \frac{\sqrt{c \delta q}}{M^{\frac{1}{4}} N^{\frac{1}{4}}\left(a_{1} a_{2}\right)^{\frac{1}{4}}} \int_{0}^{\infty} \int_{0}^{\infty} \mathcal{H}(y, z) \mathrm{e}(g(y, z, n, m)) d y d z,
$$

where $\frac{\partial^{j} \partial^{i} \mathcal{H}(y, z)}{\partial y^{j} \partial z^{i}} \ll \frac{1}{N^{j} M^{i}}, \mathcal{H}(x, y)$ is supported in $[N, 2 N] \times[M, 2 M]$, and

$$
g(y, z, n, m)=\frac{a_{1}^{2} b_{1} y}{c \delta q a_{2} b_{2}}+\frac{a_{2}^{2} b_{2} z}{c \delta q a_{1} b_{1}} \pm \frac{3 n^{\frac{1}{3}} y^{\frac{1}{3}}}{\eta_{1}} \pm \frac{3 m^{\frac{1}{3}} z^{\frac{1}{3}}}{\eta_{2}} \pm \frac{2 \sqrt{a_{1} a_{2} y z}}{c \delta q} .
$$

We note that the integration over $y, z$ above is $O(M N)$. Hence we obtain that

$$
\frac{\partial g(y, z, n, m)}{\partial y}=B_{1} \pm \frac{n^{\frac{1}{3}}}{y^{\frac{2}{3}} \eta_{1}} \pm \frac{A_{1}}{y^{\frac{1}{2}}}
$$

and

$$
\frac{\partial g(y, z, n, m)}{\partial z}=B_{2} \pm \frac{m^{\frac{1}{3}}}{z^{\frac{2}{3}} \eta_{2}} \pm \frac{A_{2}}{z^{\frac{1}{2}}}
$$

where $A_{1}=\frac{\sqrt{a_{1} a_{2} z}}{c \delta q}$ and $A_{2}=\frac{\sqrt{a_{1} a_{2} y}}{c \delta q}$. We will divide into three cases to consider.

Case 2.1: $a_{2}^{\frac{3}{2}} b_{2} M^{\frac{1}{2}} \geq 4 a_{1}^{\frac{3}{2}} b_{1} N^{\frac{1}{2}}$. For this case, we have that $\left|\frac{A_{1}}{y^{\frac{1}{2}}} \pm B_{1}\right| \asymp \frac{A_{1}}{y^{\frac{1}{2}}}$, and $\left|\frac{A_{2}}{z^{\frac{1}{2}}} \pm B_{2}\right| \asymp B_{2}$. By similar arguments to case 2 of section 8.1.2, we consider the ranges $n \asymp\left(A_{1} \eta_{1}\right)^{3} N^{\frac{1}{2}}$ and $m \asymp\left(B_{2} \eta_{2}\right)^{3} M^{2}$. By the same arguments as in Section 8.1, we note that

$$
\left(A \eta_{1}^{3}\right) N^{\frac{1}{2}} \ll\left(\frac{\sqrt{a_{1}}}{\delta q^{\frac{1}{2}}}\right)^{3} N^{\frac{1}{2}} \ll \frac{q^{\varepsilon}}{\delta^{3}}, \quad\left(B \eta_{2}\right)^{3} M^{2} \ll \frac{\left(a_{2}^{2} b_{2}\right)^{3} M^{2}}{\delta^{3} q^{3}} \ll \frac{q^{\varepsilon}}{\delta^{3}}
$$

and there are no terms of this from unless $N \gg q^{\frac{3}{2}} / a_{1}^{3}, M \gg \frac{q^{3 / 2}}{\left(a_{2}^{2} b_{2}\right)^{3 / 2}}$ and $\delta \ll q^{\varepsilon}$. Hence the contribution from these terms to $E_{5,4}^{+}(\mathbf{a}, \mathbf{b}, M, N)$ is

$$
M^{\frac{1}{6}} N^{\frac{1}{6}} \sum_{\delta<q^{\varepsilon}} \sum_{c \ll \frac{\sqrt{a_{1} a_{2} M N}}{\delta q}} \frac{1}{\eta_{1}^{\frac{1}{2}} \eta_{2}^{\frac{1}{2}}} \sum_{h_{1} \mid \eta_{1}} \sqrt{h_{1}} \sum_{\substack{n \ll q^{\varepsilon} \\ h_{1}^{2} \mid n}} \frac{1}{n^{\frac{1}{3}}} \sum_{h_{2} \mid \eta_{1}} \sqrt{h_{2}} \sum_{\substack{m \ll q^{\varepsilon} \\ h_{2}^{2} \mid m}} \frac{1}{m^{\frac{1}{3}}} \ll a_{1}^{\frac{1}{2}} a_{2}^{\frac{1}{2}} q^{\frac{1}{2}+\varepsilon} .
$$


Case 2.2: $a_{1}^{\frac{3}{2}} b_{1} N^{\frac{1}{2}} \geq 4 a_{2}^{\frac{3}{2}} b_{2} M^{\frac{1}{2}}$. For this case, we do the same calculation as in case 2.1 and obtain that the contribution is also $O\left(a_{1}^{\frac{1}{2}} a_{2}^{\frac{1}{2}} q^{\frac{1}{2}+\varepsilon}\right)$.

Case 2.3: $\frac{1}{4} a_{1}^{\frac{3}{2}} b_{1} N^{\frac{1}{2}}<a_{2}^{\frac{3}{2}} b_{2} M^{\frac{1}{2}}<4 a_{1}^{\frac{3}{2}} b_{1} N^{\frac{1}{2}}$. For this case, we have that $\frac{A_{1}}{y^{\frac{1}{2}}} \asymp B_{1}$, and $\frac{A_{2}}{z^{\frac{1}{2}}} \asymp B_{2}$. By similar arguments to case 2 of Section 8.1.2, we can focus on the ranges $n \asymp\left(A_{1} \eta_{1}\right)^{3} N^{\frac{1}{2}}$ and $m \asymp\left(A_{1} \eta_{1}\right)^{3} N^{\frac{1}{2}}$. The contribution from these terms to $E_{5,4}^{+}(\mathbf{a}, \mathbf{b}, M, N)$ is then $\ll a_{1}^{\frac{1}{2}} a_{2}^{\frac{1}{2}} q^{\frac{1}{2}+\varepsilon}$.

\section{Conclusion of the proof of Theorem 2.5}

Recall that from (4.1) and (4.2), we want to evaluate

$$
H(0 ; \boldsymbol{\alpha}, \boldsymbol{\beta}) \mathcal{M}_{6}(q)=\mathscr{M}_{1}(q ; \boldsymbol{\alpha}, \boldsymbol{\beta})+\mathscr{M}_{1}(q ; \boldsymbol{\beta}, \boldsymbol{\alpha}) .
$$

By $(4.3)$, we see that $\mathscr{M}_{1}(q ; \boldsymbol{\alpha}, \boldsymbol{\beta})=\mathscr{D}(q ; \boldsymbol{\alpha}, \boldsymbol{\beta})+\mathscr{K}(q ; \boldsymbol{\alpha}, \boldsymbol{\beta})$, and in Lemma 4.1, we showed that

$$
\mathscr{D}(q ; \boldsymbol{\alpha}, \boldsymbol{\beta})=H(0 ; \boldsymbol{\alpha}, \boldsymbol{\beta}) \int_{-\infty}^{\infty} \mathcal{M}(q ; \boldsymbol{\alpha}+i t, \boldsymbol{\beta}+i t) d t+O\left(q^{-3 / 4+\varepsilon}\right),
$$

which is one of the twenty main terms of the asymptotic formula. Then we decomposed $\mathscr{K}(q ; \boldsymbol{\alpha}, \boldsymbol{\beta})$ as $\mathscr{K}_{M}(q ; \boldsymbol{\alpha}, \boldsymbol{\beta})+\mathscr{K}_{E}(q ; \boldsymbol{\alpha}, \boldsymbol{\beta})$. We proved in Section 5.1 that $\mathscr{K}_{E}(q ; \boldsymbol{\alpha}, \boldsymbol{\beta}) \ll$ $q^{-1 / 2+\varepsilon}$, and then using Voronoi Summation formula, we extracted another nine main terms of the asymptotic formula from $\mathscr{K}_{M}(q ; \boldsymbol{\alpha}, \boldsymbol{\beta})$ with an error term $O\left(q^{-\frac{1}{4}+\varepsilon}\right)$ (see Proposition 6.1 and 6.2, $\S 7, \S 8$ and Appendix A). As briefly discussed in $\S 4$, those terms correspond to $\mathcal{M}(q ; \pi(\boldsymbol{\alpha})+i t, \pi(\boldsymbol{\beta})+i t)$, where $\pi$ is the transposition $\left(\alpha_{i}, \beta_{j}\right)$ for $i=1,2,3$ in $S_{6} / S_{3} \times S_{3}$. Hence $\mathscr{M}_{1}(q ; \boldsymbol{\alpha}, \boldsymbol{\beta})$ gives ten main terms the desired asymptotic formula, and similarly the remaining ten terms comes from $\mathscr{M}_{1}(q ; \boldsymbol{\beta}, \boldsymbol{\alpha})$.

Therefore combining everything together, we have that

$H(0 ; \boldsymbol{\alpha}, \boldsymbol{\beta}) \mathcal{M}_{6}(q)=H(0 ; \boldsymbol{\alpha}, \boldsymbol{\beta}) \int_{-\infty}^{\infty} \sum_{\pi \in S_{6} / S_{3} \times S_{3}} \mathcal{M}(q ; \pi(\boldsymbol{\alpha})+i t, \pi(\boldsymbol{\beta})+i t) d t+O\left(q^{-1 / 4+\varepsilon}\right)$.

If $\left|\alpha_{i}-\beta_{j}\right| \gg q^{-\varepsilon}$ for all $1 \leq i, j \leq 3$, then $H(0 ; \boldsymbol{\alpha}, \boldsymbol{\beta}) \gg q^{-\varepsilon}$ and we immediately get

$$
\mathcal{M}_{6}(q)=\int_{-\infty}^{\infty} \sum_{\pi \in S_{6} / S_{3} \times S_{3}} \mathcal{M}(q ; \pi(\boldsymbol{\alpha})+i t, \pi(\boldsymbol{\beta})+i t) d t+O\left(q^{-1 / 4+\varepsilon}\right) .
$$

However, since all expressions above - including the term bounded by $O\left(q^{-1 / 2+\varepsilon}\right)$ - are analytic in the $\alpha_{i}$ and $\beta_{j}$, we see that this in fact holds in general.

\section{Appendix A. Comparing the main term of $R_{\alpha_{1}, \beta_{1}}$ And $\mathcal{M}(q ; \pi(\boldsymbol{\alpha}), \pi(\boldsymbol{\beta}))$}

To finish the proof of Proposition 6.1, we will show that the local factor at prime $p$ of the Euler product of $\zeta\left(1-\alpha_{1}+\beta_{1}\right) \mathcal{M}_{\alpha_{1}, \beta_{1}}(0)$ is the same as the one in $\mathcal{A} \mathcal{Z}\left(\frac{1}{2} ; \pi(\boldsymbol{\alpha}), \pi(\boldsymbol{\beta})\right)$, where $(\pi(\boldsymbol{\alpha}), \pi(\boldsymbol{\beta}))=\left(\beta_{1}, \alpha_{1}, \alpha_{2} ; \alpha_{1}, \beta_{2}, \beta_{3}\right)$ and $\mathcal{M}_{\alpha_{1}, \beta_{1}}(s)$ is defined as in (7.10). To simplify the presentation, we will work within the ring of formal Dirichlet series, so that we need not worry about convergence issues in this section. Indeed, if we show 
that $\zeta\left(1-\alpha_{1}+\beta_{1}\right) \mathcal{M}_{\alpha_{1}, \beta_{1}}(0)$ is the same as $\mathcal{A Z}\left(\frac{1}{2} ; \pi(\boldsymbol{\alpha}), \pi(\boldsymbol{\beta})\right)$ as formal series, then they must have the same region of absolute convergence. Thus, as analytic functions, they agree on the region of absolute convergence, and so must be the same by analytic continuation. Note that we have already verified that there is a non-empty open region of absolute convergence at the end of $\S 7$.

For notational convenience, $\boldsymbol{\alpha}_{2,3}=\left(\alpha_{2}, \alpha_{3}\right)$, and $-\boldsymbol{\beta}_{2,3}=\left(-\beta_{2},-\beta_{3}\right)$ in this section.

A.1. Euler product at prime $p$ of $\mathcal{A Z}\left(\frac{1}{2} ; \pi(\boldsymbol{\alpha}), \pi(\boldsymbol{\beta})\right)$. We start from rearranging the sums in $\mathcal{A Z}(s ; \pi(\boldsymbol{\alpha}), \pi(\boldsymbol{\beta}))$ by the same method as in $(2.9)$. When $\operatorname{Re}\left(s+\beta_{1}+\right.$ $\left.\alpha_{2}+\alpha_{3}\right), \operatorname{Re}\left(s-\alpha_{1}-\beta_{2}-\beta_{3}\right)>1$, we recall that from Equations (2.13) and (2.17), $\mathcal{A Z}(s ; \pi(\boldsymbol{\alpha}), \pi(\boldsymbol{\beta}))$ is

$$
\sum \sum_{\substack{a_{1}, b_{1}, a_{2}, b_{2}, m, n \geq 1 \\ a_{1} n=a_{2} m \\ a_{1} b_{1}=a_{2} b_{2} \\\left(a_{i}, q\right)=\left(b_{j}, q\right)=1}} \sum \frac{\mathscr{B}\left(a_{1}, b_{1} ; \pi(\boldsymbol{\alpha})\right)}{\left(a_{1} b_{1}\right)^{2 s}} \frac{\mathscr{B}\left(a_{2}, b_{2} ;-\pi(\boldsymbol{\beta})\right)}{\left(a_{2} b_{2}\right)^{2 s}} \frac{\sigma_{3}(n ; \pi(\boldsymbol{\alpha})) \sigma_{3}(m ;-\pi(\boldsymbol{\beta}))}{\left(a_{1} n\right)^{s}\left(a_{2} m\right)^{s}} .
$$

Using Lemma 2.1 and the proof of Lemma 2.9 and using the fact that

$$
\sigma_{3}\left(a ; \alpha_{1}, \alpha_{2}, \alpha_{3}\right)=\sum_{d f=a} d^{-\alpha_{1}} \sigma_{2}\left(f ; \alpha_{2}, \alpha_{3}\right),
$$

we see after a change of variables that

$$
\mathcal{A Z}\left(\frac{1}{2} ; \pi(\boldsymbol{\alpha}), \pi(\boldsymbol{\beta})\right)=\zeta\left(1-\alpha_{1}+\beta_{1}\right) \sum \sum_{\substack{d_{1}, d_{2}, e_{1}, e_{2} \geq 1 \\ d_{1} e_{1}=d_{2} e_{2} \\\left(d_{i} e_{i}, q\right)=1}} \sum_{1} \frac{1}{d_{1}^{1+\alpha_{2}+\alpha_{3}} d_{2}^{1-\beta_{2}-\beta_{3}}} \frac{1}{e_{1}^{1+\beta_{1}} e_{2}^{1-\alpha_{1}}} \mathcal{J}\left(e_{1}, e_{2}\right),
$$

where

$$
\mathcal{J}\left(e_{1}, e_{2}\right)=\sum_{j_{1}, j_{2} \geq 1} \frac{\sigma_{2}\left(j_{1} e_{1} ; \boldsymbol{\alpha}_{2,3}\right) \sigma_{2}\left(j_{2} e_{2} ;-\boldsymbol{\beta}_{2,3}\right)\left(j_{1}, j_{2}\right)^{1-\alpha_{1}+\beta_{1}}}{j_{1}^{1-\alpha_{1}} j_{2}^{1+\beta_{1}}}
$$

Since both $\zeta\left(1-\alpha_{1}+\beta_{1}\right) \mathcal{M}_{\alpha_{1}, \beta_{1}}(0)$ and $\mathcal{A} \mathcal{Z}\left(\frac{1}{2} ; \pi(\boldsymbol{\alpha}), \pi(\boldsymbol{\beta})\right)$ have the factor $\zeta\left(1-\alpha_{1}+\right.$ $\left.\beta_{1}\right)$, it suffices to consider only the local factor at prime $p$ of the sum over $d_{i}, e_{i}$ in (A.2). For $p \neq q$, this is

$$
\sum \sum_{\substack{\delta_{1}, \delta_{2}, \epsilon_{1}, \epsilon_{2} \geq 0 \\ \delta_{1}+\epsilon_{1}=\delta_{2}+\epsilon_{2}}} \sum_{p^{D+\epsilon_{1}+\epsilon_{2}+\epsilon_{1} \beta_{1}-\epsilon_{2} \alpha_{1}}} \sum_{k \geq 0} \frac{\mathcal{J}_{p}\left(\epsilon_{1}, \epsilon_{2}, k\right)}{p^{k}}
$$

where $p^{\delta_{i}}\left\|d_{i}, p^{\epsilon_{i}}\right\| e_{i}, p^{\iota_{i}} \| j_{i}$,

$$
D:=\delta_{1}+\delta_{2}+\delta_{1}\left(\alpha_{2}+\alpha_{3}\right)-\delta_{2}\left(\beta_{2}-\beta_{3}\right),
$$


and

$$
\begin{aligned}
\mathcal{J}_{p}\left(\epsilon_{1}, \epsilon_{2}, k\right):=\sigma_{2}\left(p^{k+\epsilon_{1}} ; \boldsymbol{\alpha}_{2,3}\right) \sigma_{2}\left(p^{k+\epsilon_{2}} ;-\boldsymbol{\beta}_{2,3}\right) & +\sum_{0 \leq \iota_{1}<k} \frac{\sigma_{2}\left(p^{\iota_{1}+\epsilon_{1}} ; \boldsymbol{\alpha}_{2,3}\right) \sigma_{2}\left(p^{k+\epsilon_{2}} ;-\boldsymbol{\beta}_{2,3}\right)}{p^{\beta_{1}\left(k-\iota_{1}\right)}} \\
& +\sum_{0 \leq \iota_{2}<k} \frac{\sigma_{2}\left(p^{k+\epsilon_{1}} ; \boldsymbol{\alpha}_{2,3}\right) \sigma_{2}\left(p^{\iota_{2}+\epsilon_{2}} ;-\boldsymbol{\beta}_{2,3}\right)}{p^{-\alpha_{1}\left(k-\iota_{2}\right)}} .
\end{aligned}
$$

For $p=q$, we have that $\delta_{i}=\epsilon_{i}=0$, and the local factor at $p$ is

$$
\sum_{k \geq 0} \frac{\mathcal{J}_{p}(0,0, k)}{p^{k}}
$$

We also comment here that when $\alpha_{i}=\beta_{i}=0$ for $i=1,2,3$, using $\sigma_{2}\left(p^{k}\right)=k+1$ in (A.4), (A.6), (A.7) and some straightforward calculation, we derive that the local factor at $p \neq q$ of $\mathcal{A} \mathcal{Z}\left(\frac{1}{2} ; 0,0\right)$ is

$$
\left(1-\frac{1}{p}\right)^{-9} C_{p},
$$

where $C_{p}$ is defined in (1.4), and the local factor at $q$ is

$$
\left(1-\frac{1}{q}\right)^{-5}\left(1+\frac{4}{q}+\frac{1}{q^{2}}\right) .
$$

This explains the presence of the arithmetic factor in our Conjecture 1.1, as in the work $[2]$.

A.2. The Euler product at $p$ of $\mathcal{M}_{\alpha_{1}, \beta_{1}}(0)$. First, by the definition of $\mathscr{B}\left(a, b ; \alpha_{1}, \alpha_{2}, \alpha_{3}\right)$ in $(2.6), \mathcal{G}(c, \mathbf{a}, \mathbf{b})$ in $(7.9), \sum^{\#} \mathscr{G}_{\mathbf{a}, \mathbf{b}}(s ; h, b, c, \gamma, g)$ in (7.6), Equation (A.1), and a change of variables, we obtain that $\mathcal{M}_{\alpha_{1}, \beta_{1}}(0)$ can be re-written as

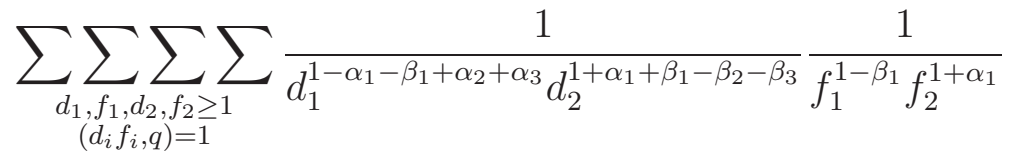

$$
\begin{aligned}
& \cdot \sum_{\substack{h \geq 1 \\
h \mid u_{1} u_{2}}} \frac{\mu(h)}{h^{\alpha_{1}-\beta_{1}}} \sum_{\substack{b \geq 1 \\
\left(b, u_{1} u_{2}\right)=1}} \frac{\mu(b)}{b^{\alpha_{1}-\beta_{1}}} \sum_{c \geq 1} \frac{c}{c^{\alpha_{1}-\beta_{1}}} \sum_{\gamma \mid c} \frac{1}{\gamma^{\alpha_{1}-\beta_{1}}} \sum_{g \mid \frac{c}{\gamma}} \frac{\mu(g)}{g^{\alpha_{1}-\beta_{1}}} \prod_{\substack{p \mid c \\
p \nmid b h \gamma}}\left(1-\frac{1}{p}\right) \\
& \cdot \sum_{n \geq 1} \sum_{a_{1} \mid f_{1}} \frac{\mu\left(a_{1}\right)}{a_{1}^{\alpha_{2}+\alpha_{3}}}\left(\frac{\left(a_{1}, u_{2} c b\right)}{a_{1} u_{2} c b n}\right)^{1-\alpha_{1}} \sigma_{2}\left(\frac{u_{2} c b n}{\left(a_{1}, u_{2} c b\right)} ; \boldsymbol{\alpha}_{2,3}\right) \sigma_{2}\left(\frac{f_{1}}{a_{1}} ; \boldsymbol{\alpha}_{2,3}\right) \\
& \cdot \sum_{m \geq 1} \sum_{a_{2} \mid f_{2}} \frac{\mu\left(a_{2}\right)}{a_{2}^{-\beta_{2}-\beta_{3}}}\left(\frac{\left(a_{2}, u_{1} c b\right)}{a_{2} u_{1} c b m}\right)^{1+\beta_{1}} \sigma_{2}\left(\frac{u_{1} c b m}{\left(a_{2}, u_{1} c b\right)} ;-\boldsymbol{\beta}_{2,3}\right) \sigma_{2}\left(\frac{f_{2}}{a_{2}} ;-\boldsymbol{\beta}_{2,3}\right) \text {. }
\end{aligned}
$$


In Section 7.2, $u_{i}=\frac{a_{i} b_{i}}{\left(a_{1} b_{1}, a_{2} b_{2}\right)}$, but after changing variables, we write that $u_{i}=\frac{f_{i} d_{i}}{\left(f_{1} d_{1}, f_{2} d_{2}\right)}$. By comparing Euler products, we can show that

$\sum_{n \geq 1} \sum_{a_{1} \mid f_{1}} \frac{\mu\left(a_{1}\right)}{a_{1}^{\alpha_{2}+\alpha_{3}}}\left(\frac{\left(a_{1}, u_{2} c b\right)}{a_{1} u_{2} c b n}\right)^{1-\alpha_{1}} \sigma_{2}\left(\frac{u_{2} c b n}{\left(a_{1}, u_{2} c b\right)} ; \boldsymbol{\alpha}_{2,3}\right) \sigma_{2}\left(\frac{f_{1}}{a_{1}} ; \boldsymbol{\alpha}_{2,3}\right)=\sum_{n^{\prime} \geq 1} \frac{\sigma_{2}\left(f_{1} u_{2} c b n^{\prime} ; \boldsymbol{\alpha}_{2,3}\right)}{\left(u_{2} c b n^{\prime}\right)^{1-\alpha_{1}}}$.

We also have a similar expression for the sum over $m$ and $a_{2}$. Hence we can write $\mathcal{M}_{\alpha_{1}, \beta_{1}}(0)$ as

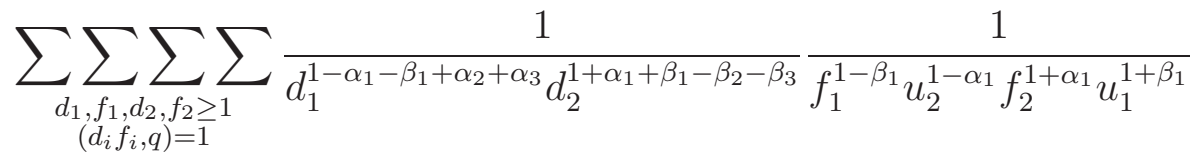

$$
\begin{aligned}
& \cdot \sum_{\substack{h \geq 1 \\
h \mid u_{1} u_{2}}} \frac{\mu(h)}{h^{\alpha_{1}-\beta_{1}}} \sum_{\substack{b \\
\left(b, u_{1} u_{2}\right)=1}} \frac{\mu(b)}{b^{2}} \sum_{c} \frac{1}{c} \sum_{\gamma \mid c} \frac{1}{\gamma^{\alpha_{1}-\beta_{1}}} \sum_{g \mid \frac{c}{\gamma}} \frac{\mu(g)}{g^{\alpha_{1}-\beta_{1}}} \prod_{\substack{p \mid c \\
p \nmid b h \gamma}}\left(1-\frac{1}{p}\right) \\
& \cdot \sum_{n, m \geq 1} \sum_{n^{1-\alpha_{1}}} \frac{\sigma_{2}\left(f_{1} u_{2} c b n ; \boldsymbol{\alpha}_{2,3}\right)}{\sigma_{2}\left(f_{2} u_{1} c b m ;-\boldsymbol{\beta}_{2,3}\right)} m^{1+\beta_{1}} .
\end{aligned}
$$

We note here that $d_{1} f_{1} u_{2}=d_{2} f_{2} u_{1}$ by the definition of $u_{1}, u_{2}$. Next, we consider the local factor at $p \neq q$ of (A.8), which is of the form

$$
\sum \sum_{\substack{\delta_{1}, \delta_{2}, \xi_{1}, \xi_{2} \geq 0 \\ \delta_{1}+\ell_{1}=\delta_{2}+\ell_{2}}} \sum_{p^{D^{\prime}\left(\ell_{1}, \ell_{2}\right)+\ell_{2} \beta_{1}-\ell_{1} \alpha_{1}-\left(\xi_{1}+\xi_{2}\right)\left(\beta_{1}-\alpha_{1}\right)}} \mathscr{L}_{p}\left(\delta_{1}, \delta_{2}, \xi_{1}, \xi_{2}\right)
$$

where $p^{\delta_{i}}\left\|d_{i}, p^{\xi_{i}}\right\| f_{i}, p^{v_{i}} \| u_{i}, \ell_{1}=\xi_{1}+v_{2}, \ell_{2}=\xi_{2}+v_{1}, \min \left\{v_{1}, v_{2}\right\}=0, D^{\prime}\left(\ell_{1}, \ell_{2}\right)=$ $D+\left(\delta_{2}-\delta_{1}\right)\left(\alpha_{1}+\beta_{1}\right)+\ell_{1}+\ell_{2}$, and $D$ is defined in (A.5). We will examine $\mathscr{L}_{p}\left(d_{1}, d_{2}, f_{1}, f_{2}\right)$ below but before that analysis, we need the following two Lemmas.

Lemma A.1. The contribution to the local factor at $p$ from

$$
\sum_{\gamma \mid c} \frac{1}{\gamma^{\alpha_{1}-\beta_{1}}} \sum_{g \mid \frac{c}{\gamma}} \frac{\mu(g)}{g^{\alpha_{1}-\beta_{1}}} \prod_{\substack{p \mid c \\ p \nmid b h \gamma}}\left(1-\frac{1}{p}\right)
$$

is 1 if $p \nmid c$ or $p \mid b h$. Otherwise, it is $1-\frac{1}{p}+\frac{1}{p^{1+\alpha_{1}-\beta_{1}}}$.

Proof. For $p \nmid c$ or $p \mid b h$, the contribution to the local factor is 1 because

$$
\sum_{\gamma \mid c} \frac{1}{\gamma^{\alpha_{1}-\beta_{1}}} \sum_{g \mid \frac{c}{\gamma}} \frac{\mu(g)}{g^{\alpha_{1}-\beta_{1}}}=\sum_{a \mid c} \frac{1}{a^{\alpha_{1}-\beta_{1}}} \sum_{g \mid a} \mu(g)=1 .
$$

Now suppose $p \mid c$ and $p \nmid b h$. Below we write $p^{c_{p}} \| c$ and $p^{\gamma_{p}} \| \gamma$. Then the contribution to the local factor at $p$ is

$$
\left(1-\frac{1}{p^{\alpha_{1}-\beta_{1}}}\right)\left(1-\frac{1}{p}\right)+\sum_{1 \leq \gamma_{p}<c_{p}} \frac{1}{p^{\gamma_{p}\left(\alpha_{1}-\beta_{1}\right)}}\left(1-\frac{1}{p^{\alpha_{1}-\beta_{1}}}\right)+\frac{1}{p^{c_{p}\left(\alpha_{1}-\beta_{1}\right)}}=1-\frac{1}{p}+\frac{1}{p^{1+\alpha_{1}-\beta_{1}}} .
$$


Lemma A.2. Let

$$
\mathscr{P}\left(\ell_{1}, \ell_{2}\right):=\sum_{c_{p} \geq 0} \sum_{n_{p}, m_{p} \geq 0} \frac{1}{p^{c_{p}}} \frac{\sigma_{2}\left(p^{\ell_{1}+n_{p}+c_{p}} ; \boldsymbol{\alpha}_{2,3}\right)}{p^{n_{p}\left(1-\alpha_{1}\right)}} \frac{\sigma_{2}\left(p^{\ell_{2}+m_{p}+c} ;-\boldsymbol{\beta}_{2,3}\right)}{p^{m_{p}\left(1+\beta_{1}\right)}} .
$$

Then

$$
\mathscr{P}\left(\ell_{1}, \ell_{2}\right)=\sum_{k \geq 0} \frac{\mathcal{J}_{p}\left(\ell_{1}, \ell_{2}, k\right)}{p^{k}}+\frac{p^{\alpha_{1}-\beta_{1}}}{p^{2}} \mathscr{P}\left(\ell_{1}+1, \ell_{2}+1\right)
$$

where $\mathcal{J}_{p}\left(\ell_{1}, \ell_{2}, k\right)$ is defined as in (A.6).

Proof. We have

$$
\begin{aligned}
\mathscr{P}\left(\ell_{1}, \ell_{2}\right) & =\sum_{c_{p} \geq 0} \frac{\sigma_{2}\left(p^{\ell_{1}+c_{p}} ; \boldsymbol{\alpha}_{2,3}\right) \sigma_{2}\left(p^{\ell_{2}+c_{p}} ;-\boldsymbol{\beta}_{2,3}\right)}{p^{c_{p}}}+\sum_{c_{p} \geq 0, n_{p} \geq 1} \frac{\sigma_{2}\left(p^{\ell_{1}+n_{p}+c_{p}} ; \boldsymbol{\alpha}_{2,3}\right) \sigma_{2}\left(p^{\ell_{2}+c_{p}} ;-\boldsymbol{\beta}_{2,3}\right)}{p^{c_{p}+n_{p}-n_{p} \alpha_{1}}} \\
& +\sum_{c_{p} \geq 0} \sum_{m_{p} \geq 1} \frac{\sigma_{2}\left(p^{\ell_{1}+c_{p}} ; \boldsymbol{\alpha}_{2,3}\right) \sigma_{2}\left(p^{\ell_{2}+m_{p}+c_{p}} ;-\boldsymbol{\beta}_{2,3}\right)}{p^{c_{p}+m_{p}+m_{p} \beta_{1}}}+\frac{p^{\alpha_{1}-\beta_{1}}}{p^{2}} \mathscr{P}\left(\ell_{1}+1, \ell_{2}+1\right) \\
& =\sum_{k \geq 0} \frac{\mathcal{J}_{p}\left(\ell_{1}, \ell_{2}, k\right)}{p^{k}}+\frac{p^{\alpha_{1}-\beta_{1}}}{p^{2}} \mathscr{P}\left(\ell_{1}+1, \ell_{2}+1\right),
\end{aligned}
$$

after some arrangement.

Now we examine $\mathscr{L}_{p}\left(\delta_{1}, \delta_{2}, \xi_{1}, \xi_{2}\right)$ which we separate into two cases below.

Case 1: $\delta_{1}+\xi_{1}=\delta_{2}+\xi_{2}$. For this case, we have $v_{1}=v_{2}=0$, so $\xi_{i}=\ell_{i}$. Hence $u_{1} u_{2}=1$ and $p \nmid h$. From Lemma A.1, we then obtain that

$$
\mathscr{L}_{p}\left(\delta_{1}, \delta_{2}, \xi_{1}, \xi_{2}\right)=\mathscr{P}\left(\ell_{1}, \ell_{2}\right)+\frac{1}{p^{2}}\left(p^{\beta_{1}-\alpha_{1}}-1\right) \mathscr{P}\left(\ell_{1}+1, \ell_{2}+1\right)-\frac{1}{p^{2}} \mathscr{P}\left(\ell_{1}+1, \ell_{2}+1\right) .
$$

From Lemma A.2, $\mathscr{L}_{p}\left(d_{1}, d_{2}, f_{1}, f_{2}\right)$ can be written as

$$
\begin{aligned}
& \sum_{k \geq 0} \frac{\mathcal{J}_{p}\left(\ell_{1}, \ell_{2}, k\right)}{p^{k}}+\frac{1}{p^{2}}\left(p^{\alpha_{1}-\beta_{1}}-2+p^{\beta_{1}-\alpha_{1}}\right) \mathscr{P}\left(\ell_{1}+1, \ell_{2}+1\right) \\
& =\sum_{k \geq 0} \frac{\mathcal{J}_{p}\left(\ell_{1}, \ell_{2}, k\right)}{p^{k}}+\frac{1}{p^{2}}\left(p^{\alpha_{1}-\beta_{1}}-2+p^{\beta_{1}-\alpha_{1}}\right) \sum_{k \geq 0} \frac{\mathcal{J}_{p}\left(\ell_{1}+1, \ell_{2}+1, k\right)}{p^{k}} \\
& \quad+\frac{p^{\alpha_{1}-\beta_{1}}}{p^{4}}\left(p^{\alpha_{1}-\beta_{1}}-2+p^{\beta_{1}-\alpha_{1}}\right) \mathscr{P}\left(\ell_{1}+2, \ell_{2}+2\right) \\
& =\sum_{k \geq 0} \frac{1}{p^{k}}\left\{\mathcal{J}_{p}\left(\ell_{1}, \ell_{2}, k\right)+\left(p^{\alpha_{1}-\beta_{1}}-2+p^{\beta_{1}-\alpha_{1}}\right) \sum_{1 \leq m_{p} \leq\left\lfloor\frac{k}{2}\right\rfloor} p^{\left(m_{p}-1\right)\left(\alpha_{1}-\beta_{1}\right)} \mathcal{J}_{p}\left(\ell_{1}+m_{p}, \ell_{2}+m_{p}, k-2 m_{p}\right)\right\}
\end{aligned}
$$


Case 2: $\delta_{1}+\xi_{1} \neq \delta_{2}+\xi_{2}$. For this case, $v_{1}+v_{2} \geq 1$. So $p \mid u_{1} u_{2}$, and $b_{p}=0$, where $p^{b_{p}} \| b$. By Lemma A.1 and A.2, we have

$$
\begin{aligned}
& \mathscr{L}_{p}\left(\delta_{1}, \delta_{2}, \xi_{1}, \xi_{2}\right)=\left(1-\frac{1}{p^{\alpha_{1}-\beta_{1}}}\right) \mathscr{P}\left(\ell_{1}, \ell_{2}\right)-\frac{1}{p^{2}}\left(1-\frac{1}{p^{\alpha_{1}-\beta_{1}}}\right) \mathscr{P}\left(\ell_{1}+1, \ell_{2}+1\right) \\
& =\left(1-\frac{1}{p^{\alpha_{1}-\beta_{1}}}\right)\left\{\sum_{k \geq 0} \frac{\mathcal{J}_{p}\left(\ell_{1}, \ell_{2}, k\right)}{p^{k}}+\frac{1}{p^{2}}\left(p^{\alpha_{1}-\beta_{1}}-1\right) \mathscr{P}\left(\ell_{1}+1, \ell_{2}+1\right)\right\} \\
& =\left(1-\frac{1}{p^{\alpha_{1}-\beta_{1}}}\right)\left\{\sum_{k \geq 0} \frac{\mathcal{J}_{p}\left(\ell_{1}, \ell_{2}, k\right)}{p^{k}}+\frac{1}{p^{2}}\left(p^{\alpha_{1}-\beta_{1}}-1\right) \sum_{k \geq 0} \frac{\mathcal{J}_{p}\left(\ell_{1}+1, \ell_{2}+1, k\right)}{p^{k}}\right. \\
& \left.+\frac{p^{\alpha_{1}-\beta_{1}}}{p^{4}}\left(p^{\alpha_{1}-\beta_{1}}-1\right) \mathscr{P}\left(\ell_{1}+2, \ell_{2}+2\right)\right\} \\
& =\left(1-\frac{1}{p^{\alpha_{1}-\beta_{1}}}\right) \sum_{k \geq 0} \frac{1}{p^{k}}\left\{\mathcal{J}_{p}\left(\ell_{1}, \ell_{2}, k\right)\right. \\
& \left.+\left(p^{\alpha_{1}-\beta_{1}}-1\right) \sum_{1 \leq m_{p} \leq\left\lfloor\frac{k}{2}\right\rfloor} p^{\left(m_{p}-1\right)\left(\alpha_{1}-\beta_{1}\right)} \mathcal{J}_{p}\left(\ell_{1}+m_{p}, \ell_{2}+m_{p}, k-2 m_{p}\right)\right\} .
\end{aligned}
$$

From both cases, we obtain that (A.9) is

$$
\begin{aligned}
& \sum \sum_{\substack{\delta_{1}, \delta_{2}, \ell_{1}, \ell_{2} \geq 0 \\
\delta_{1}+\ell_{1}=\delta_{2}+\ell_{2}}} \sum_{p^{D^{\prime}\left(\ell_{1}, \ell_{2}\right)-\ell_{1} \beta_{1}+\ell_{2} \alpha_{1}}} \mathscr{L}_{p}\left(\delta_{1}, \delta_{2}, \ell_{1}, \ell_{2}\right) \\
& \left.+\sum \sum_{\substack{\delta_{1}, \delta_{2}, \ell_{1}, \ell_{2} \geq 0 \\
\delta_{1}+\ell_{1}=\delta_{2}+\ell_{2}}} \sum_{\substack{0 \leq \xi_{2}<\ell_{2} \\
\xi_{1}=\ell_{1}}} \frac{\mathscr{L}_{p}\left(\delta_{1}, \delta_{2}, \xi_{1}, \xi_{2}\right)}{p^{\left(\ell_{2}-\ell_{1}\right) \beta_{1}-\xi_{2}\left(\beta_{1}-\alpha_{1}\right)}}+\sum_{\substack{0 \leq \xi_{1}<\ell_{1} \\
\xi_{2}=\ell_{2}}} \frac{\mathscr{L}_{p}\left(\delta_{1}, \delta_{2}, \xi_{1}, \xi_{2}\right)}{p^{\left(\ell_{2}-\ell_{1}\right) \alpha_{1}-\xi_{1}\left(\beta_{1}-\alpha_{1}\right)}}\right) \frac{1}{p^{D^{\prime}\left(\ell_{1}, \ell_{2}\right)}} \\
& :=\sum_{\delta_{1}, \delta_{2} \geq 0} \sum_{\mathcal{S}_{p}}\left(\delta_{1}, \delta_{2}\right) \text {, }
\end{aligned}
$$

say. For fixed $\delta_{1}, \delta_{2}$, where $\delta_{2} \geq \delta_{1}$, we rearrange the term $\mathcal{S}_{p}\left(\delta_{1}, \delta_{2}\right)$ and obtain that 
(A.12)

$$
\begin{aligned}
S_{p}\left(\delta_{1}, \delta_{2}\right)= & \sum_{\substack{\epsilon_{1}, \epsilon_{2} \geq 0 \\
\delta_{1}+\epsilon_{1}=\delta_{2}+\epsilon_{2}}} \sum_{k \geq 0} \frac{\mathcal{J}_{p}\left(\epsilon_{1}, \epsilon_{2}, k\right)}{p^{D^{\prime}\left(\epsilon_{1}, \epsilon_{2}\right)+k}}\left\{\frac{1}{p^{\left(\epsilon_{2}-\epsilon_{1}\right) \alpha_{1}}}+\frac{1}{p^{\left(\epsilon_{2}-\epsilon_{1}\right) \beta_{1}}}-\frac{1}{p^{-\epsilon_{1} \beta_{1}+\epsilon_{2} \alpha_{1}}}\right. \\
& +\left(p^{\alpha_{1}-\beta_{1}}-2+p^{\beta_{1}-\alpha_{1}}\right) \sum_{1 \leq \ell_{2} \leq \epsilon_{2}} \frac{p^{\left(\ell_{2}-1\right)\left(\alpha_{1}-\beta_{1}\right)}}{p^{-\left(\epsilon_{1}-\ell_{2}\right) \beta_{1}+\left(\epsilon_{2}-\ell_{2}\right) \alpha_{1}}} \\
& \left.+\left(p^{\alpha_{1}-\beta_{1}}-1\right) \sum_{1 \leq \ell_{2} \leq \epsilon_{2}} p^{\left(\ell_{2}-1\right)\left(\alpha_{1}-\beta_{1}\right)}\left(\frac{1}{p^{\left(\epsilon_{2}-\epsilon_{1}\right) \alpha_{1}}}+\frac{1}{p^{\left(\epsilon_{2}-\epsilon_{1}\right) \beta_{1}}}-\frac{2 p^{\ell_{2}\left(\alpha_{1}-\beta_{1}\right)}}{p^{-\epsilon_{1} \beta_{1}+\epsilon_{2} \alpha_{1}}}\right)\right\} \\
= & \sum_{\substack{\epsilon_{1}, \epsilon_{2} \geq 0 \\
\delta_{1}+\epsilon_{1}=\delta_{2}+\epsilon_{2}}} \sum_{k \geq 0} \frac{\mathcal{J}_{p}\left(\epsilon_{1}, \epsilon_{2}, k\right)}{p^{D+\epsilon_{1}+\epsilon_{2}+k+\epsilon_{1} \beta_{1}-\epsilon_{2} \alpha_{1}}} .
\end{aligned}
$$

By similar calculation $\mathcal{S}_{p}\left(\delta_{1}, \delta_{2}\right)$ yields the same value for $\delta_{1}>\delta_{2}$. In summary from (A.9), (A.11) and (A.12), the local factor at $p$ of $\mathcal{M}_{\alpha_{1}, \beta_{1}}$ is

$$
\sum_{\substack{\epsilon_{1}, \epsilon_{2} \geq 0 \\ \delta_{1}+\epsilon_{1}=\delta_{2}+\epsilon_{2}}} \sum_{k \geq 0} \frac{\mathcal{J}_{p}\left(\epsilon_{1}, \epsilon_{2}, k\right)}{p^{D+\epsilon_{1}+\epsilon_{2}+k+\epsilon_{1} \beta_{1}-\epsilon_{2} \alpha_{1}}},
$$

which is the same as the local factor at $p$ of $\mathcal{A} \mathcal{Z}\left(\frac{1}{2} ; \pi(\boldsymbol{\alpha}), \pi(\boldsymbol{\beta})\right)$ in (A.4), as desired.

For $p=q$, we use similar arguments, with $\delta_{i}=\epsilon_{i}=0$, so that the Euler factor is

$$
\sum_{k \geq 0} \frac{\mathcal{J}_{p}(0,0, k)}{p^{k}}
$$

which is the same as the local factor at $q$ of $\mathcal{A Z}\left(\frac{1}{2} ; \pi(\boldsymbol{\alpha}), \pi(\boldsymbol{\beta})\right)$ in (A.7). This completes the proof of Proposition 6.1.

\section{Appendix B. Voronoi Summation}

In this section, we state the Voronoi Summation formula for the shifted $k$-divisor function defined in (2.5). The proof of this formula is essentially the same as the proof by Ivic of the Voronoi Summation formula for the $k$-divisor function in [11], so we will state the results and refer the reader to Ivic for detailed proofs.

Let $\omega$ be a smooth compactly supported function. For $\boldsymbol{\alpha}=\left(\alpha_{1}, \ldots, \alpha_{k}\right)$, let $\sigma_{k}(n ; \boldsymbol{\alpha})=$ $\sigma_{k}\left(n ; \alpha_{1}, \ldots, \alpha_{k}\right)$. Define

$$
S\left(\frac{a}{c}, \boldsymbol{\alpha}\right)=\sum_{n=1}^{\infty} \sigma_{k}(n ; \boldsymbol{\alpha}) e\left(\frac{a n}{c}\right) \omega(n),
$$

where $(a, c)=1$, and the Mellin transform of $\omega$

$$
\tilde{\omega}(s)=\int_{0}^{\infty} \omega(x) x^{s-1} d x .
$$


Since $\omega$ was chosen to be from the Schwarz class, $\tilde{\omega}$ is entire and decays rapidly on vertical lines. We have the Mellin inversion formula

$$
\omega(n)=\frac{1}{2 \pi i} \int_{(c)} \tilde{\omega}(s) \frac{d s}{n^{s}}
$$

where $c$ is any vertical line. Let

$$
\begin{aligned}
& A_{k}\left(m, \frac{a}{c}, \boldsymbol{\alpha}\right) \\
& \quad=\frac{1}{2} \sum_{\substack{m_{1}, \ldots, m_{k} \geq 1 \\
m_{1} \ldots m_{k}=m}} m_{1}^{\alpha_{1}} \ldots m_{k}^{\alpha_{k}} \sum_{a_{1}(\bmod c)} \ldots \sum_{a_{k}(\bmod c)}\left\{\mathrm{e}\left(\frac{a a_{1} \ldots a_{k}+\mathbf{a} \cdot \mathbf{m}}{c}\right)+\mathrm{e}\left(\frac{-a a_{1} \ldots a_{k}+\mathbf{a} \cdot \mathbf{m}}{c}\right)\right\},
\end{aligned}
$$

and

$$
\begin{aligned}
& B_{k}\left(m, \frac{a}{c}, \boldsymbol{\alpha}\right) \\
& \quad=\frac{1}{2} \sum_{\substack{m_{1}, \ldots, m_{k} \geq 1 \\
m_{1} \ldots m_{k}=m}} m_{1}^{\alpha_{1}} \ldots m_{k}^{\alpha_{k}} \sum_{a_{1}(\bmod c)} \ldots \sum_{a_{k}(\bmod c)}\left\{\mathrm{e}\left(\frac{a a_{1} \ldots a_{k}+\mathbf{a} \cdot \mathbf{m}}{c}\right)-\mathrm{e}\left(\frac{-a a_{1} \ldots a_{k}+\mathbf{a} \cdot \mathbf{m}}{c}\right)\right\} .
\end{aligned}
$$

Moreover, we define

$$
G_{k}(s, n, \boldsymbol{\alpha})=\frac{c^{k s}}{\pi^{k s} n^{s}}\left(\prod_{\ell=1}^{k} \frac{\Gamma\left(\frac{s-\alpha_{\ell}}{2}\right)}{\Gamma\left(\frac{1-s+\alpha_{\ell}}{2}\right)}\right), \quad H_{k}(s, n, \boldsymbol{\alpha})=\frac{c^{k s}}{\pi^{k s} n^{s}}\left(\prod_{\ell=1}^{k} \frac{\Gamma\left(\frac{1+s-\alpha_{\ell}}{2}\right)}{\Gamma\left(\frac{2-s+\alpha_{\ell}}{2}\right)}\right)
$$

and for $0<\sigma<\frac{1}{2}-\frac{1}{k}+\frac{\sum_{\ell=1}^{k} \operatorname{Re}\left(\alpha_{\ell}\right)}{k}$, we let

$$
U_{k}(x ; \boldsymbol{\alpha})=\frac{1}{2 \pi i} \int_{(\sigma)} \prod_{\ell=1}^{k} \frac{\Gamma\left(\frac{s-\alpha_{\ell}}{2}\right)}{\Gamma\left(\frac{1-s+\alpha_{\ell}}{2}\right)} \frac{d s}{x^{s}}, \quad V_{k}(x ; \boldsymbol{\alpha})=\frac{1}{2 \pi i} \int_{(\sigma)} \prod_{\ell=1}^{k} \frac{\Gamma\left(\frac{1+s-\alpha_{\ell}}{2}\right)}{\Gamma\left(\frac{2-s+\alpha_{\ell}}{2}\right)} \frac{d s}{x^{s}} .
$$

We note that by Stirling's formula, both integrals for $U_{k}$ and $V_{k}$ are absolutely convergent.

Finally, we define the Dirichlet series to be

$$
D_{k}\left(s, \frac{a}{c}, \boldsymbol{\alpha}\right)=\sum_{n} \frac{\sigma_{k}(n ; \boldsymbol{\alpha}) e\left(\frac{a n}{c}\right)}{n^{s}},
$$

which converges absolutely for $\operatorname{Re} s>1$. We have that

$$
\begin{aligned}
D_{k}\left(s, \frac{a}{c}, \boldsymbol{\alpha}\right) & =\sum_{n_{1}, \ldots, n_{k}} \frac{e\left(\frac{a n_{1} n_{2} \ldots n_{k}}{c}\right)}{n_{1}^{s+\alpha_{1}} \ldots n_{k}^{s+\alpha_{k}}} \\
& =\frac{1}{c^{k s+\alpha_{1}+\ldots+\alpha_{k}}} \sum_{a_{1}, \ldots, a_{k} \bmod c} e\left(\frac{a a_{1} \ldots a_{k}}{c}\right) \prod_{j=1}^{k} \zeta\left(s+\alpha_{j}, \frac{a_{j}}{c}\right),
\end{aligned}
$$


where $\zeta\left(s, \frac{a}{c}\right)$ is the Hurwitz zeta function defined for $\operatorname{Re} s>1$ as

$$
\zeta\left(s, \frac{a}{c}\right)=\sum_{n=0}^{\infty} \frac{1}{\left(n+\frac{a}{c}\right)^{s}} .
$$

The Hurwitz zeta function may be analytically continued to all of $\mathbb{C}$ except for a simple pole at $s=1$. Therefore, $D_{k}\left(s, \frac{a}{c}\right)$ can be analytically continued to all of $\mathbb{C}$ except for a simple pole at $s=1-\alpha_{j}$ for $j=1, . ., k$.

Theorem B.1. With notations as above and $(a, c)=1$ and $\alpha_{i} \ll \frac{1}{1000 k \log (|c|+100)}$, we have

$$
\begin{aligned}
S\left(\frac{a}{c}, \boldsymbol{\alpha}\right) & =\sum_{\ell=1}^{k} \operatorname{Res}_{s=1-\alpha_{\ell}} \tilde{\omega}(s) D_{k}\left(s, \frac{a}{c}, \boldsymbol{\alpha}\right) \\
& +\frac{\pi^{k / 2+\alpha_{1}+\ldots+\alpha_{k}}}{c^{k+\alpha_{1}+\ldots+\alpha_{k}}} \sum_{n=1}^{\infty} A_{k}\left(n, \frac{a}{c}, \boldsymbol{\alpha}\right) \int_{0}^{\infty} \omega(x) U_{k}\left(\frac{\pi^{k} n x}{c^{k}} ; \boldsymbol{\alpha}\right) d x \\
& +i^{3 k} \frac{\pi^{k / 2+\alpha_{1}+\ldots+\alpha_{k}}}{c^{k+\alpha_{1}+\ldots+\alpha_{k}}} \sum_{n=1}^{\infty} B_{k}\left(n, \frac{a}{c}, \boldsymbol{\alpha}\right) \int_{0}^{\infty} \omega(x) V_{k}\left(\frac{\pi^{k} n x}{c^{k}} ; \boldsymbol{\alpha}\right) d x .
\end{aligned}
$$

We refer the reader to the proof of Theorem 2 in [11] for details. Next, we collect properties of $A_{3}(n, a / c, \boldsymbol{\alpha}), B_{3}(n, a / c, \boldsymbol{\alpha}), U_{3}(x ; \boldsymbol{\alpha})$ and $V_{3}(x ; \boldsymbol{\alpha})$. These are useful for bounding error terms of $\mathcal{M}_{6}(q)$.

Lemma B.2. Let $a, n, \gamma$ be integers such that $(a, \gamma)=1$. Moreover $A_{3}(n, a / c, \boldsymbol{\alpha})$, $B_{3}(n, a / c, \boldsymbol{\alpha})$ are defined as in (B.1) and (B.2). Then

$$
\begin{gathered}
\sum_{n_{1} n_{2} n_{3}=n} n_{1}^{\alpha_{1}} n_{2}^{\alpha_{2}} n_{3}^{\alpha_{3}} \sum_{r_{1}, r_{2}, r_{3}} \sum_{(\bmod \gamma)} \sum \mathrm{e}\left(\frac{a r_{1} r_{2} r_{3}+r_{1} n_{1}+r_{2} n_{2}+r_{3} n_{3}}{\gamma}\right) \\
=\gamma \sum_{h\left|\gamma, h^{2}\right| n} h \Delta(n, h, \gamma) S\left(\frac{n}{h^{2}},-\bar{a}, \frac{\gamma}{h}\right),
\end{gathered}
$$

where $\Delta(n, h, \gamma)$ is a divisor function satisfying $\Delta(n, h, \gamma) \ll(\gamma n)^{\varepsilon}$. Moreover,

$$
A_{3}\left(n, \frac{a}{\gamma}, \boldsymbol{\alpha}\right) \ll(\gamma n)^{\varepsilon} \gamma^{\frac{3}{2}} \sum_{h\left|\gamma, h^{2}\right| n} \sqrt{h}, \quad B_{3}\left(n, \frac{a}{\gamma}, \boldsymbol{\alpha}\right) \ll(\gamma n)^{\varepsilon} \gamma^{\frac{3}{2}} \sum_{h\left|\gamma, h^{2}\right| n} \sqrt{h} .
$$

The proof of this lemma can be found in Equations (8.7)-(8.9) in [11].

Lemma B.3. If $U(x ; \boldsymbol{\alpha}):=U_{3}(x ; \boldsymbol{\alpha})$ and $V(x ; \boldsymbol{\alpha}):=V_{3}(x ; \boldsymbol{\alpha})$, as defined in (B.3), and $\alpha_{i} \ll \frac{1}{1000 k \log (|c|+100)}$, then for any $0<\varepsilon<1 / 6$ and $x>0$, we have

$$
U(x ; \boldsymbol{\alpha}) \ll x^{\varepsilon}, \quad V(x ; \boldsymbol{\alpha}) \ll x^{\varepsilon} .
$$

Moreover for any fixed integer $K \geq 1$ and $x \geq x_{0}>0$,

$$
U(x ; \boldsymbol{\alpha})=\sum_{j=1}^{K} \frac{c_{j} \cos \left(6 x^{\frac{1}{3}}\right)+d_{j} \sin \left(6 x^{\frac{1}{3}}\right)}{x^{\frac{j}{3}+\frac{\alpha_{1}+\alpha_{2}+\alpha_{3}}{3}}}+O\left(\frac{1}{x^{\frac{K+1}{3}+\frac{\alpha_{1}+\alpha_{2}+\alpha_{3}}{3}}}\right),
$$




$$
V(x ; \boldsymbol{\alpha})=\sum_{j=1}^{K} \frac{e_{j} \cos \left(6 x^{\frac{1}{3}}\right)+f_{j} \sin \left(6 x^{\frac{1}{3}}\right)}{x^{\frac{j}{3}+\frac{\alpha_{1}+\alpha_{2}+\alpha_{3}}{3}}}+O\left(\frac{1}{x^{\frac{K+1}{3}+\frac{\alpha_{1}+\alpha_{2}+\alpha_{3}}{3}}}\right),
$$

with suitable constants $c_{j}, \ldots, f_{j}$, and $c_{1}=0, d_{1}=-\frac{2}{\sqrt{3 \pi}} e_{1}=-\frac{2}{\sqrt{3 \pi}}, f_{1}=0$.

The proof of this lemma is a minor modification of the proof of Lemma 3 in [11].

\section{ACKNOWLEDGMENT}

Vorrapan Chandee acknowledges support from Coordinating Center for Thai Government Science and Technology Scholarship Students (CSTS) and National Science and Technology Development Agency (NSTDA) grant of year 2014. Part of this work was done while the first author was visiting Mathematical Institute, University of Oxford she is grateful for their kind hospitality.

\section{REFERENCES}

[1] V. Chandee and Xiannan Li, The eighth moment of Dirichlet L-functions, Adv. Math. 259 (2014), 339-375.

[2] J.B. Conrey, D. Farmer, J.Keating, M. Rubinstein, and N.Snaith, Integral moments of Lfunctions, Proc. London Math. Soc. 91 (2005), 33-104.

[3] J.B. Conrey and A. Ghosh, A conjecture for the sixth power moment of the Riemann zetafunction. Internat. Math. Res. Notices 1998, no. 15, 775 - 780.

[4] J.B. Conrey and S.M. Gonek, High moments of the Riemann zeta-function, Duke Math. J. 107 (2001), no. 3, $577-604$.

[5] J.B. Conrey, H. Iwaniec and K. Soundararajan, The sixth power moment of Dirichlet Lfunctions, Geometric and Functional Analysis, October 2012, Volume 22, Issue 5, 1257-1288.

[6] H. Davenport, Multiplicative Number Theory, vol.74, Springer-Verlag (GTM), New York, 2000.

[7] G. Djanković, The sixth moment of the family of $\Gamma_{1}(q)$-automorphic L-functions, Arch. Math. 97 (2011), $535-547$.

[8] A. Diaconu, D. Goldfeld, and J. Hoffstein, Multiple Dirichlet series and moments of zeta and L-functions. Compositio Math. 139 (2003), 297-360.

[9] I. S. Gradshteyn and I. M. Ryzhik, Table of Integrals, Series, and Products, Edited by A.Jeffrey and D. Zwillinger. Academic Press, New York, 7th edition, 2007.

[10] A. Harper, Sharp conditional bounds for moments of the Riemann zeta function. Preprint: http://arxiv.org/abs/1305.4618.

[11] A. Ivić, On the ternary additive divisor problem and the sixth moment of the zeta-function, Sieve methods, exponential sums, and their applications in number theory (Cardiff, 1995), London Math. Soc. Lecture Note Ser., vol. 237, Cambridge Univ. Press, Cambridge, 1997, 205 - 243.

[12] H. Iwaniec and E. Kowalski, Analytic Number Theory, vol. 53, American Mathematical Society Colloquium Publications, Rhode Island, 2004.

[13] H.Iwaniec and Xiaoqing Li, The orthogonality of Hecke eigenvalues, Compos. Math. 143 (2007), p.541-565.

[14] N. Katz and P. Sarnak, Random matrices, Frobenius eigenvalues, and monodromy. American Mathematical Society Colloquium Publications, 45. American Mathematical Society, Providence, RI, 1999.

[15] J.P. Keating and N.C. Snaith, Random matrix theory and $\zeta\left(\frac{1}{2}+i t\right)$, Comm. Math. Phys. 214 (2000), 57-89.

[16] J.P. Keating and N.C. Snaith, Random matix theory and L-functions at $s=1 / 2$ Comm. Math. Phys. 214 (2000), 91 - 110. 
[17] Z. Rudnick and K. Soundararajan, Lower bounds for moments of L-functions, Proc. Natl. Acad. Sci. USA 102 (2005), 6837-6838.

[18] F. Oberhettinger, Tables of Bessel transforms, Springer, Berlin, 1972.

[19] K. Soundararajan, Moments of the Riemann zeta-function, Ann. of Math. (2) 170 (2009) 981 993.

[20] K. Soundararajan, The fourth moment of Dirichlet L-functions, Analytic number theory, 239246, Clay Math. Proc., 7, Amer. Math. Soc., Providence, RI, 2007.

[21] E.C. Titchmarsh, The Theory of the Riemann Zeta-function, Oxford University Press, New York, 1986.

[22] G. N. Watson, A treatise on the theory of Bessel functions, Cambridge University Press, Cambridge 1944.

Department of Mathematics, Burapha University, 169 Long-Hard Bangsaen rd, SaenSuk, Mueng, Chonburi, Thailand, 20131

E-mail address: vorrapan@buu.ac.th

University of Oxford, Andrew Wiles Building, Radcliffe Observatory Quarter, WoodSTOCK ROAD, OXFORD, UK, OX2 6GG

Current address: Department of Mathematics, Kansas State University, 138 Cardwell Hall, Manhattan, Kansas, USA, 66506

E-mail address: xiannan@math.ksu.edu 